I N T ER N ATIONAL MONETARY FUND

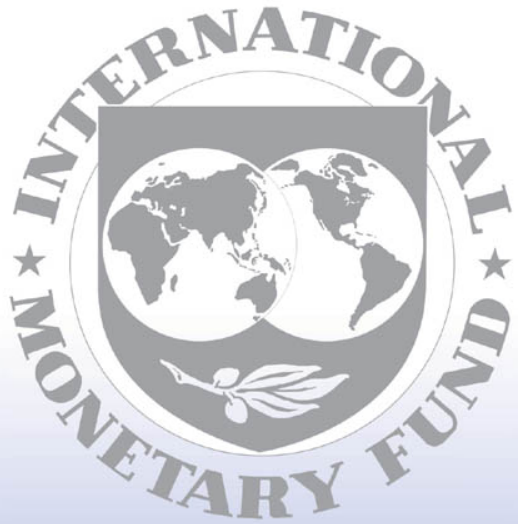

Staff

Country

Reports 


\section{Democratic Republic of the Congo: Fourth Review Under the Three-Year Arrangement Under the Poverty Reduction and Growth Facility, Requests for Waiver of Performance Criteria and Additional Interim Assistance Under the Enhanced Initiative for Heavily Indebted Poor Countries-Staff Report; Staff Statement; Press Release on the Executive Board Discussion; and Statement by the Executive Director for the Democratic Republic of the Congo}

In the context of the fourth review under the three-year arrangement under the Poverty Reduction and Growth Facility, requests for a waiver of performance criteria and additional interim assistance under the enhanced Initiative for Heavily Indebted Poor Countries, with the Democratic Republic of the Congo, the following documents have been released and are included in this package:

- $\quad$ the staff report for the fourth review under the three-year arrangement under the Poverty Reduction and Growth Facility, requests for waiver of performance criteria and additional interim assistance under the enhanced Initiative for Heavily Indebted Poor Countries, prepared by a staff team of the IMF, following discussions that ended on May 29, 2004, with the officials of the Democratic Republic of the Congo on economic developments and policies. Based on information available at the time of these discussions, the staff report was completed on June 28, 2004. The views expressed in the staff report are those of the staff team and do not necessarily reflect the views of the Executive Board of the IMF.

- $\quad$ a staff statement of July 12, 2004 updating information on recent developments.

a Press Release summarizing the views of the Executive Board as expressed during its July 12, 2004 discussion of the staff report that completed the request and review.

- $\quad$ a statement by the Executive Director for the Democratic Republic of the Congo.

The documents listed below have been or will be separately released.

Interim Poverty Reduction Strategy Paper Preparation Status Report

Joint Staff Assessment of the Interim Poverty Reduction Strategy Preparation Status Report Letter of Intent sent to the IMF by the authorities of the Democratic Republic of the Congo* Memorandum of Economic and Financial Policies by the authorities of the

Democratic Republic of the Congo*

Technical Memorandum of Understanding*

* May also be included in Staff Report

The policy of publication of staff reports and other documents allows for the deletion of market-sensitive information.

To assist the IMF in evaluating the publication policy, reader comments are invited and may be sent by e-mail to publicationpolicy@imf.org

Copies of this report are available to the public from

International Monetary Fund $\bullet$ Publication Services

$70019^{\text {th }}$ Street, N.W. • Washington, D.C. 20431

Telephone: (202) 623-7430 • Telefax: (202) 623-7201

E-mail: publications@imf.org•Internet: http://www.imf.org

Price: $\$ 15.00$ a copy

International Monetary Fund

Washington, D.C.

CInternational Monetary Fund. Not for Redistribution 
This page intentionally left blank 


\section{INTERNATIONAL MONETARY FUND}

\section{DEMOCRATIC REPUBLIC OF THE CONGO}

\section{Fourth Review Under the Three-Year Arrangement Under the Poverty Reduction and Growth Facility, Requests for Waiver of Performance Criteria and Additional Interim Assistance Under the Enhanced Initiative for Heavily Indebted Poor Countries}

Prepared by the African Department

(In consultation with the Finance, Fiscal Affairs, Legal, Monetary and Financial Systems, Policy Development and Review, and Statistics Departments)

Approved by Menachem Katz and Anthony Boote

June 28, 2004

Executive Summary

I. Introduction. .4

II. Recent Political and Security Developments $\underline{5}$

III. Performance Under the PRGF-Supported Program in 2003 and the First Quarter of 2004

IV. Report on the Policy Discussions and the Program for 2004 $\underline{10}$

A. Macroeconomic Framework for 2004 ................................................................

B. Structural Policies ....................................................................................15

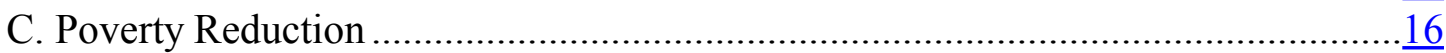

D. Administrative Capacity Building, Technical Assistance, and Statistical Issues....16

V. PRGF Arrangement Monitoring …………….........................................................

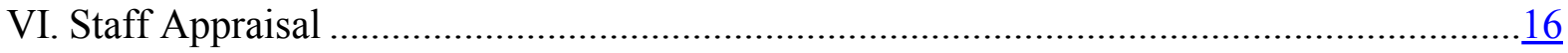

Boxes

1. The National Disarmament, Demobilization and Reintegration (DDR) Program .......... $\underline{5}$

2. Territorial Organization and Fiscal Decentralization...............................................14 
Figures

1. Selected Fiscal and Monetary Indicators, 1998-2004 …............................................

2. Selected Monetary Data, 2003-04 ......................................................................

3. Exchange Rate Indices, January 1996-March 2004 ..............................................19

Tables

1. Fund Position During the PRGF Arrangement, 2002-05 ......................................20

2. Proposed Schedule of Disbursements Under the PRGF Arrangement, 2002-05 ............21

3. Selected Economic and Financial Indicators, 2002-07 ….....................................22

4A. Summary of Central Government Financial Operations, 2002-07 (In Congo francs) ....24

4B. Summary of Central Government Financial Operations, 2002-07 (In percent of GDP).26

5. Monetary Survey, 2002-04 ........................................................................28

6. Balance of Payments Summary, 2002-07...........................................................29

7. Nominal Stock of External Public Debt and Net Present Value of

External Public Debt by Creditor Group, End-2003 …...................................... $\frac{31}{32}$

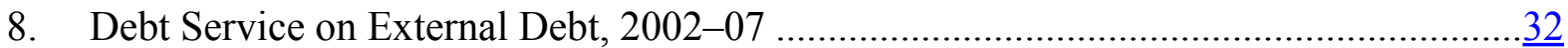

9. External Financing Requirements and Sources, 2002-07 ........................................33

10. Democratic Republic of the Congo and Sub-Saharan Africa: Selected Poverty and

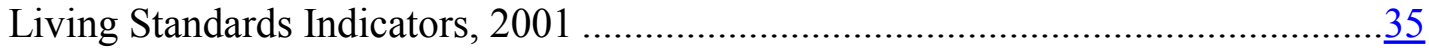

Appendices

I. Letter of Intent.....................................................................................

Attachment I. Memorandum on Economic and Financial Policies for 2004 ............ $\underline{40}$

Table 1. Selected Economic and Financial Indicators, 2002-07 .......................

Table 2A. Monthly Treasury Cash-Flow Plan, 2003 ......................................... $\frac{65}{66}$

Table 2B. Monthly Treasury Cash-Flow Plan, 2004 ....................................... $\frac{66}{68}$

Table 3. Action Plan of the Central Bank of the Congo ....................................

Table 4. Quarterly Quantitative Performance Criteria and Indicative Targets, 2003-04 ................................................................ 73

Table 5. Prior Actions and Structural Performance

Criteria and Benchmarks, 2004 ............................................................. 74

Attachment II. Technical Memorandum of Understanding .................................. $\frac{76}{83}$

Annex I. Definition of External Debt...................................................... $\frac{83}{84}$

Annex II. Definition of Poverty-Reducing Spending.................................... $\frac{84}{86}$

Annex III. Budget-Tracking Statements ................................................... $\frac{86}{87}$

II. Relations with the Fund ........................................................................ $\frac{87}{92}$

III. Relations with the World Bank Group...............................................................92

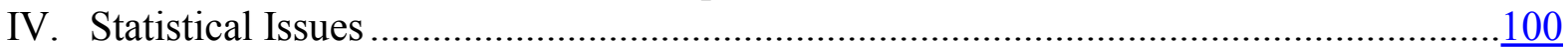




\section{Executive Summary}

Discussions. Discussions on the Fourth Review under the Poverty Reduction and Growth Facility (PRGF) arrangement were held in Kinshasa during May 15-29, 2004. The mission met with, inter alia, President Kabila, Vice President Bemba, in charge of economic and financial affairs, Vice President Ruberwa, in charge of political affairs, the President of the National Assembly, the Ministers of Finance, Budget, Economic Affairs, Health, Education, Civil Service, and Planning, the full Council of Ministers, the Governor of the Central Bank of the Congo (BCC), the President's Economic Advisor, and representatives of civil society, the international community and the press.

The staff team. The mission comprised Messrs. Clément (Head), Gons, (both AFR), Mati (EPFAD), Ioannou (PDR), Ms. Lê (Research Officer, AFR) and Ms. Jean-Baptiste (Senior Administrative Assistant, AFR), and it was assisted by Mr. Kouwenaar, the Fund's Senior Resident Representative in Kinshasa. Mr. Horton from the World Bank participated in the mission, as did Mr. Guetat, Advisor to the Executive Director for the DRC.

Political situation. Progress has continued toward the reunification of the country despite increased tensions in the eastern part, illustrated by the recent temporary take-over of the town of Bukavu by former rebel combatants. Such events, which so far have been successfully dealt with, may recur, especially in the period leading up to the elections in 2005, and will test the strength of the Government's unity and the support of the international community. The forthcoming Regional Peace Conference for the Great Lakes Region in the fall of 2004 could be an important avenue to stabilize the situation and lead to better control of military expenditure in the region.

PRGF arrangement. A three-year arrangement of SDR 580 million (108.8 percent of quota) was approved on June 12, 2002. Four tranches totaling SDR 500 million have been disbursed, and another SDR 26.7 million would become available at the completion of this review.

Enhanced HIPC Initiative decision point. The Executive Board approved the DRC's reaching the decision point on July 23, 2003. The World Bank Board took the same decision one day later. The authorities are requesting an additional annual tranche of IMF interim assistance under the enhanced HIPC Initiative.

Program implementation. Overall performance under the program was satisfactory and at endMarch 2004, 8 out of the 11 quantitative performance criteria were observed. The 3 that were not, were mainly on account of delays in foreign aid disbursements. The structural performance criterion was met. Economic growth has been higher than envisaged and inflation has decelerated more rapidly than programmed. As expected, the end-2003 fiscal objectives were missed by less than 1 percent of GDP; however fiscal performance at end-March 2004 was much better than programmed, both on the revenue and on the expenditure side.

Program for 2004. Prudent fiscal and monetary policies will continue to be implemented in the context of the floating exchange rate system. Structural and sectoral measures will be deepened and progressively implemented in the entire reunified territory.

Program risks. The situation in the east continues to represent a risk, particularly to the achievement of fiscal targets, the financing of foreign-financed projects, and the reduction in poverty. 


\section{INTRODUCTION}

\section{Discussions on the Fourth Review under the Poverty Reduction and Growth} Facility (PRGF) were held in Kinshasa during May 15-29, 2004. In the attached letter of intent dated June 24, 2004, signed by His Excellency President Joseph Kabila (Appendix I), and in the memorandum on economic and financial policies (MEFP), the authorities review political and economic developments during 2003 and the first quarter of 2004, and outline their policies for the rest of 2004. They request waivers for the nonobservance of 3 of the 11 end-March 2004 quantitative performance criteria, mainly due to delays in external disbursements and fiscal slippages in 2003 that were partly offset by supplementary measures in early 2004 (MEFP, Table 4). The structural performance criterion for end-March and the two structural benchmarks for February 2004 have been met, while the two March 2004 structural benchmarks, i.e. the submission to Parliament of the new customs code and the selection of an international firm to conduct an external audit of the diamond company (MIBA), have been modified into a prior action for the completion of this review and a structural performance criterion for September 2004, respectively.

\section{At the conclusion of the Third Review under the PRGF arrangement on} March 3, 2004, Executive Directors commended the authorities for the broadly satisfactory implementation of the PRGF-supported program. Directors welcomed the inauguration of the Government of National Unity in July 2003, which marked the formal completion of the peace and reunification process initiated in early 2001. They cautioned that the Democratic Republic of the Congo (DRC) faces considerable challenges, including the achievement of effective reunification through the demobilization and reintegration of excombatants, the creation of a national army and police, and the adoption and implementation of a decentralization law. They stressed that timely and well-coordinated support from the donor community will be needed to help achieve these goals.

\section{The Fund and World Bank staffs have been cooperating closely in assisting the}

DRC. Regarding donor support, in November 2003, Paris Club creditors agreed to top up debt relief to Cologne terms in the context of the enhanced Heavily Indebted Poor Countries (HIPC) Initiative and the topping up has become effective for all creditors, with the exception of the United States. One Paris Club creditor, Switzerland, has already cancelled the entire debt, while seven others ${ }^{1}$ have indicated their preparedness to do so at the completion point. In addition, four creditors have allowed the suspension of debt service during the interim period. Regarding non-Paris Club creditors, the authorities have signed 13 agreements with commercial creditors (about 40 percent of commercial debt). In December 2003, the African Development Bank (AfDB) approved an economic recovery and reunification support

\footnotetext{
${ }^{1}$ Austria, Belgium, France, Italy, the Netherlands, Norway, and the United Kingdom.
} 
operation of about US\$60 million. At a meeting in Kinshasa in June 2004, donors confirmed their pledges for 2004 (about US\$1 billion).

\section{Recent Political And Security Developments}

4. Overall, significant progress has been made toward effective reunification. New provincial governors and vice-governors have been nominated - a precondition for the effective administration of the country's 11 provinces. The national Disarmament, Demobilization, and Reintegration (DDR) Program has been finalized with World Bank support (Box 1). In addition, a calendar has been drawn up for a constitutional referendum, and local, legislative, and presidential elections in 2005. Funding for the elections (US\$284 million), will be provided mainly by the European Union (EU) and the United Nations.

\section{Box 1. The National Disarmament, Demobilization and Reintegration (DDR) Program}

In May 2004, IDA approved a US\$100 million grant to support the government's efforts to demobilize 150,000-200,000 ex-combatants. The program, which is expected to start in September 2004, is phased over three years.

- The program was developed within the framework of the Multi-Country Demobilization and Reintegration Program (MDRP) for the greater Great Lakes region. Recently, MDRP partners endorsed a US\$100 million Trust Fund grant to complement the IDA grant.

- The program includes criteria to (i) verify whether ex-combatants belong to an eligible armed group; and (ii) whether they are eligible for integration into the army. Those who do not qualify for integration and who volunteer to demobilize will benefit from the DDR program.

- Orientation centers will be opened at the provincial level. Eligible ex-combatants will receive, in three installments, a transitional safety net allowance totaling US\$350, supporting them for a period of six to nine months. Further, they will benefit from a range of reintegration packages providing training and/or equipment.

- Health screening and HIV/AIDS prevention measures will be provided during the demobilization and reintegration phase.

5. The security situation in the east remains volatile despite the increased presence of the United Nations Observation Mission in the Congo (MONUC), whose 10,800 troops have a mandate to use force under Article 7 of the UN Charter. The takeover of the border 
town of Bukavu by ex-combatants (reportedly supported by another country), illustrates the on-going volatility. Growing resentment by the population about the perceived ineffective response from MONUC prompted large demonstrations, particularly in Kinshasa. ${ }^{2}$

Meanwhile, the ex-combatants have withdrawn and the national army has regained control of Bukavu. A Regional Peace Conference for the Great Lakes Region is scheduled to start in the fall of 2004.

\section{Performance Under the PRGF-Supported Program in 2003 And the First QUARTER OF 2004}

\section{Overall, the PRGF-supported program has been implemented satisfactorily.} Economic growth is estimated to have accelerated to 5.6 percent in 2003, reflecting an improved business climate and an increase in investment. Inflation has decelerated more quickly than envisaged in 2003 and the cumulative rate at end-May 2004 was in line with the program (Figure 1 and Table 3). Since January 2004, prices of petroleum products have been raised gradually to reflect international prices. The demand for money has continued to

Figure 1. Democratic Republic of the Congo: Selected Fiscal and Monetary Indicators, 1998-2004 1/
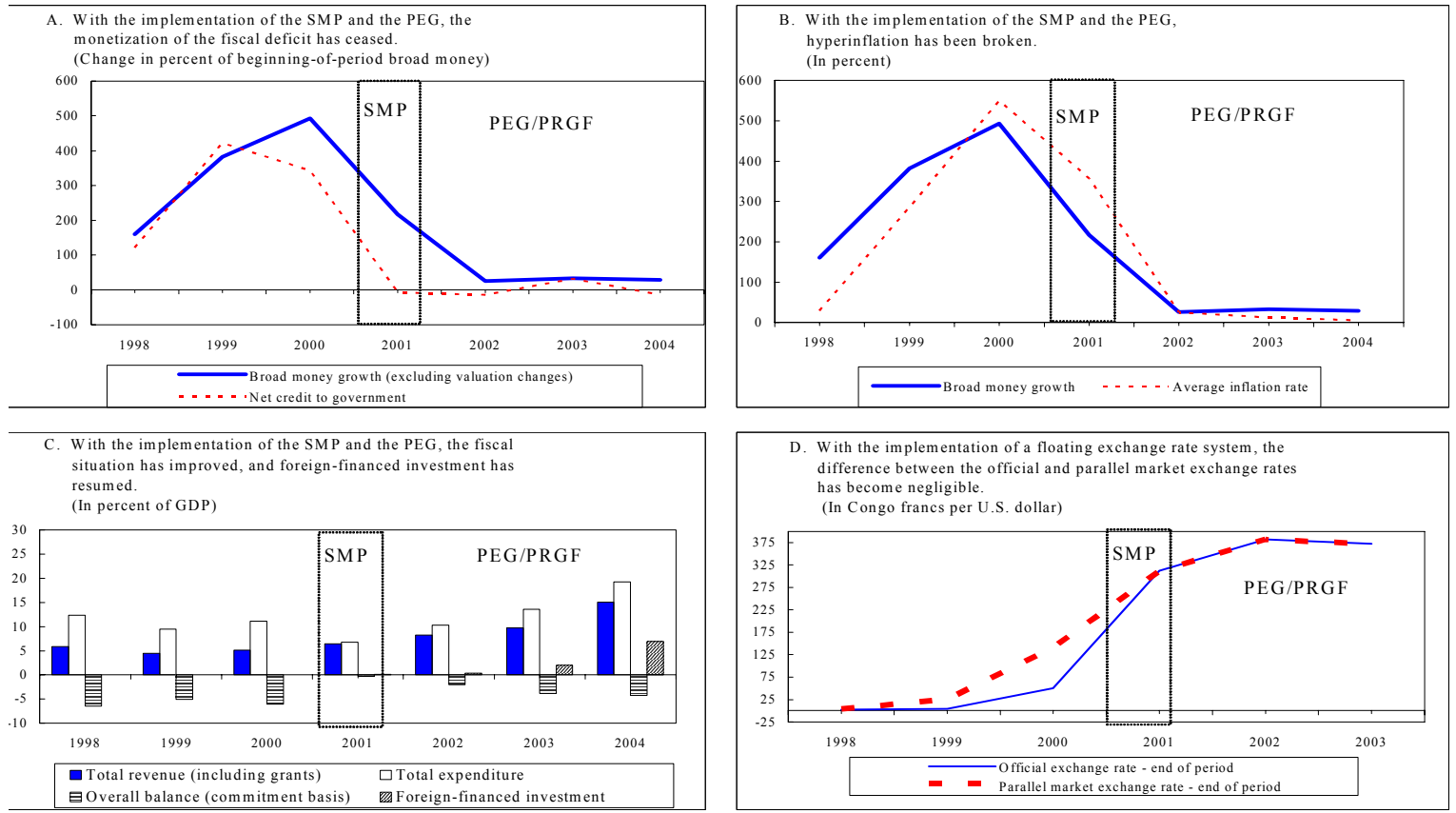

Sources: Congolese authorities; and IMF staff estimates and projections.

$1 /$ The staff-monitored program (SMP) (June 2001-March 2002). The Government Economic Program (PEG) is supported by an arrangement under the Poverty Reduction and Growth Facility (PRGF) (April 2002-July 2005).

${ }^{2}$ On June 5, 2004, the UN raised the security risk level for Kinshasa from Phase II to Phase III. 
increase and the Congo franc (CGF) has stabilized against the U.S. dollar. The real effective exchange rate depreciated by about 20 percent over the past 12 months, reflecting the decline of the U.S. dollar vis à vis the Euro. The external current account showed a stronger improvement than expected due to an increase in exports and net transfers.

7. As anticipated at the time of the third review, the fiscal objectives for 2003 have been missed by less than 1 percent of GDP. Total revenue (excluding grants) was lower than anticipated ( 0.5 percent of GDP) reflecting delays in the adoption of legislative texts related to the tariff and tax reform laws, the granting of tax exemptions, and delays in tax payments (MEFP, para. 3). Total expenditure, including larger net losses of the BCC, was 0.5 percent of GDP higher than envisaged, mainly owing to higher current primary expenditure and foreign-financed investment. The former exceeded the target by 0.3 percent of GDP associated with the creation of new civil institutions envisaged in the Transitional Constitution (MEFP, para. 3). The programmed shift toward pro-poor spending was largely achieved while the share of security expenditure was in line with the program. The domestic primary balance (on a cash basis) showed a deficit of 0.2 percent of GDP instead of the programmed 0.5 percent surplus, and the consolidated overall balance on a cash basis showed a higher deficit than programmed (MEFP, para. 3, and Table 2A). Against this background, net bank credit to the government (NCG), before adjustment for net external nonproject disbursements, was missed by 0.8 percent of GDP.

Pro-Poor and Security Spending

(In percent of GDP, unless otherwise indicated)

\begin{tabular}{lccccc}
\hline & \multicolumn{2}{c}{2003} & & \multicolumn{2}{c}{2004} \\
\cline { 2 - 3 } & $\begin{array}{c}\text { Prog. (Country } \\
\text { Report No. 04/97) }\end{array}$ & Est. ${ }^{1}$ & & $\begin{array}{c}\text { Prog. (Country } \\
\text { Report No. 04/97) }\end{array}$ & Rev. Prog \\
\hline Security & 2.1 & 2.1 & & 2.0 & 2.1 \\
Pro-poor $^{2}$ & 1.2 & 1.5 & & 6.3 & 6.6 \\
$\begin{array}{l}\text { Pro-poor (in percent of total } \\
\text { expenditure) }\end{array}$ & 9.1 & 11.7 & & 35.7 & 34.3 \\
\hline
\end{tabular}

${ }^{1}$ EU grant-related expenditures in 2003 ( 0.5 percent of GDP) are not incorporated as their classification is not available.

${ }^{2}$ Uses new pro-poor definition, including infrastructure.

8. Fiscal performance for the first quarter of 2004 was better than originally programmed both on the revenue and expenditure sides, given the supplementary measures implemented in early 2004 (MEFP, para. 5 and Table 2B). In light of this, the 
decrease in net credit to the government, before adjustment, was 0.2 percent of GDP higher than programmed. ${ }^{3}$

9. Further progress has been achieved in reforming tax policy and strengthening the tax and customs administrations, despite some delays linked to weak administrative capacity and the civil service strike of February 2004 (MEFP, paras. 6, 7 and 8). The legislative texts necessary for the completion of the March 2003 tariff and tax reform laws were finally approved by Parliament in May 2004. The new customs code will be submitted to Parliament in June. The reform of the Customs and Excise Office (OFIDA) is being implemented satisfactorily, with good progress in streamlining the customs clearance process. In addition, the Large Taxpayers Unit (LTU) is now independent in its functioning, including its audit operations.

\section{Good progress has also been made in strengthening public expenditure}

management (PEM) (MEFP, para. 10). The new expenditure procedures aimed at restoring and streamlining the entire expenditure chain, including commitment, verification, payment order and payment, are now operational. All expenditures that bypassed the new procedures for the first four months of 2004 have now been processed. Finally, the audits of the 2001 and 2002 budget executions were submitted to Parliament while good progress has been made on establishing a simplified double-entry accounting framework by end-June 2004.

\section{Monetary} policy has remained prudent. Money supply increased faster than nominal GDP in 2003 and the first quarter of 2004. The decline in velocity by 10 percent reflects the increased demand for the CGF and foreign currency deposits (Table 5). The BCC successfully introduced higher denomination banknotes (of CGF 200 and CGF 500). However, whereas it had CGF notes at its disposal and the demand for CGF was manifest-

\footnotetext{
${ }^{3}$ Without the delay in external disbursements, the NCG performance criterion for the October 2003-March 2004 period would have been met.
} 
reflecting the drop in inflationary expectations and the need for CGF in the reunified provinces - the BCC issued relatively small amounts and hesitated to purchase foreign exchange in the market. More active purchases of foreign exchange by the BCC would have helped to meet its net foreign assets (NFA) target at end-March 2004, without undermining price stability. Taking into account lower-than-expected nonproject foreign disbursements, the end-March performance criterion on its cumulative NFA was missed by US\$77 million (MEFP, Table 4). ${ }^{4}$ Since demand for broad money and base money grew in line with projections, the under performance on the BCC's NFA was matched by an overrun of its net domestic assets, reflecting higher-than-programmed NCG and an increase in other items net. The latter is a cause of concern addressed by the external audit of the end-March 2004 performance criteria, which provided recommendations to avoid increases in suspense and provincial liaison accounts. Credit to the private sector (mainly in U.S. dollars) grew strongly in 2003 and the first quarter of 2004, albeit from a low base (MEFP, paras. 13 and 14).

\section{In line with the drop in inflation, the BCC lowered its refinancing rate in steps} from 20 percent at mid-September 2003 to 8 percent since November 2003, and commercial banks' lending rates (in CGF) fell from an average of 50 percent to 25 percent.

\section{The BCC continued to implement its comprehensive action plan, with good} progress in the preparation of a new computerized accounting system, the management of international reserves, the strengthening of banking supervision, and the preparation of a bank-wide computerization plan (MEFP, para.15 and Table 3). The restructuring of the banking system advanced well with the approval by the BCC, in March 2004, of the restructuring plans of 7 banks and the decision to liquidate 2 private banks, in addition to 4 private and 3 public banks already in liquidation (MEFP, para. 16).

\section{Regarding the DRC's obligations under Article VIII, sections 2 (a), 3, and 4 of} the Fund's Articles of Agreement, the authorities have started discussions to eliminate the remaining restriction arising from the payments agreement under the Economic Community of the Great Lakes Countries (CEPGL). The staff is assessing whether the authorities' actions have been successful in eliminating the multiple currency practice arising from the payments agreement with Zimbabwe (MEFP, para. 19). On external trade policy, the authorities have eliminated the quantitative restriction on imports of printed fabric. ${ }^{5}$ The government has also

\footnotetext{
${ }^{4}$ However, during the period October 2003-March 2004, the accumulation of NFA by commercial banks was higher than envisaged largely from new foreign currency deposits.

${ }^{5}$ With an overall IMF trade restrictiveness index of 2 (with 10 being the most restrictive) the DRC's trade regime has been among the more liberal of those in the Southern African Development Community (SADC).
} 
started the process to adhere to OHADA (Organization for the Harmonization of Business Law in Africa).

\section{With the help of the World Bank, and despite some delays, good progress has} been made in implementing structural reforms (MEFP, paras. 20-43). All public enterprises have been audited, and restructuring plans for the most important ones are being prepared. Government net cross arrears with the domestic private sector (US\$840 million) have been audited. Measures have been taken to improve governance; notably the draft law on combating money laundering and the financing of terrorism has been submitted to Parliament and the draft anti-corruption law will be submitted in June 2004. However, the operations of the new Mining Registry (cadastre minier) have not been fully in line with the mining code and measures are being taken to ensure fuller transparency. Similar measures are being taken in the forestry sector.

\section{Report on the Policy Discussions ANd the Program for 2004}

\section{The medium-term macroeconomic framework for 2004-07 has been updated to}

take into account (i) the cost of the elections; (ii) the effective reunification of the country; (iii) the cost of the DDR program; (iv) the more favorable debt relief that goes beyond the enhanced HIPC Initiative; (v) anticipated external assistance; and (vi) revised estimates of the impact of the changing international environment, including higher oil prices. ${ }^{6}$

17. The macroeconomic objectives for the period 2004-07 include (i) an average real GDP growth of about 6.8 percent, implying a per capita increase of about 3.8 percent; (ii) an average annual inflation rate of 5 percent; (iii) a sharp increase in gross investment associated with reconstruction needs and the

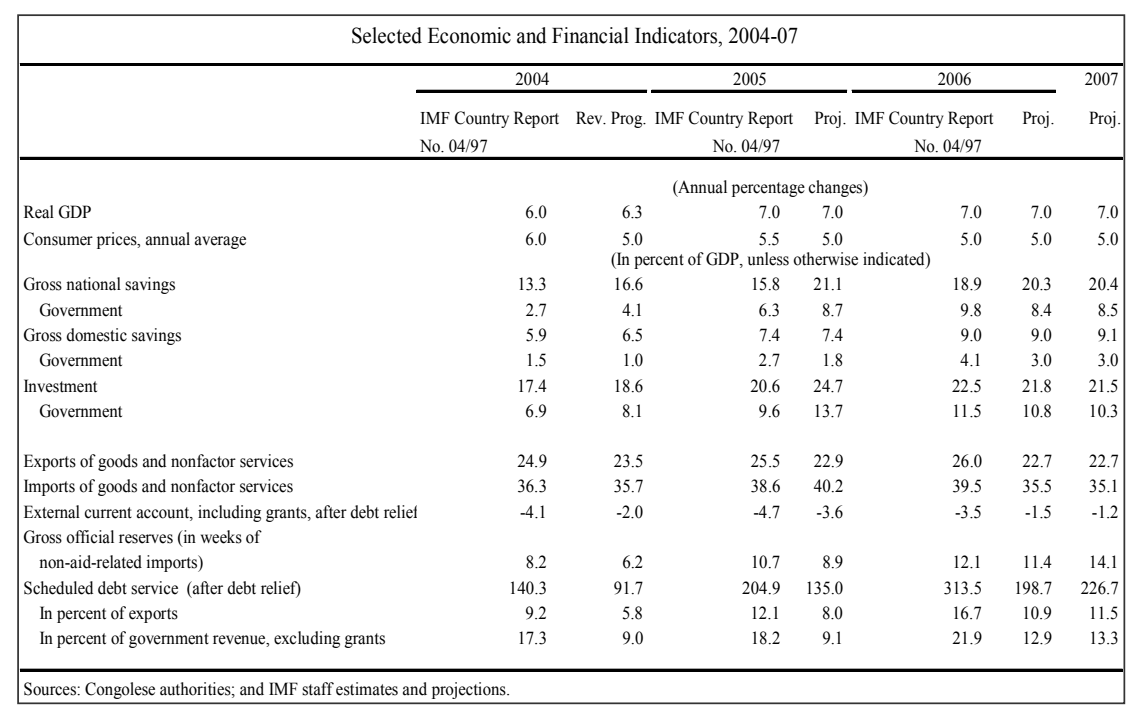

\footnotetext{
${ }^{6}$ It is estimated that a US\$ 5 increase in the price of oil leads to an increase of: (i) 3.3 percent in the value of total exports; (ii) 0.7 percent in the value of total imports; (iii) 0.2 percent of GDP in oil export-related government revenue; and (iv) 0.3 percent of GDP in retail tax-related government revenue. The direct impact on inflation is estimated at 0.6 percent.
} 
rebound in activity; and (iv) a gradual increase in gross official reserves to about 14 weeks of non-aid-related imports of goods and nonfactor services (Table 3). Debt sustainability is better than envisaged at the time of the decision point, especially in light of the commitments by a number of Paris Club creditors to provide debt relief beyond what is envisaged under the enhanced HIPC Initiative (MEFP, para. 18). The NPV of debt to exports ratio is expected to decline to 110 percent in 2006, the assumed timing of the completion point, compared with 125 percent originally envisaged. The program is, in principle, financed over the medium term, through multilateral and bilateral disbursements (Table 9).

\section{A. Macroeconomic Framework for 2004}

18. Economic growth has been raised to 6.3 percent, while the average rate of inflation has been revised downward to 5 percent. Investment would increase by 6 percentage points of GDP as a result of the acceleration of foreign-financed investment; while national saving is to increase by 3 percentage points, resulting from a rise in government savings and net external transfers. Taking into account the higher debt relief, the BCC's gross international reserves are targeted to double to six weeks of non-aid-related imports.

\section{Fiscal policy}

19. Fiscal policy for $\mathbf{2 0 0 4}$ will remain prudent while continuing the reorientation of expenditure toward pro-poor spending (MEFP, para. 45). The authorities have prepared a supplementary budget law, to be approved by the government before end-June 2004, which is consistent with the approved 2004 budget, except for including (i) the demobilization of 50,000 soldiers instead of the 150,000 previously estimated; (ii) the cost of elections preparation; (iii) the cost of the domestically financed liquidation of private commercial banks; and (iv) payment of net cross-arrears with the domestic private sector, financed by the World Bank.

\section{Achievement of the 2004 fiscal objectives will be based on the continued} implementation of revenue enhancing measures and better control of expenditure (MEFP, para. 46). Total revenue (excluding grants) is expected to reach 9.2 percent of GDP while total expenditure (on a commitment basis) is projected at 19.3 percent of GDP in 2004 . The wage bill will increase by 1 percent of GDP reflecting (i) a civil service wage increase (as of April 1, 2004) of 20 percent (30 percent for primary and secondary school teachers) and the doubling of some transportation allowances; ${ }^{7}$ (ii) the cost related to the de facto

\footnotetext{
${ }^{7}$ Because of the high unemployment rate and the fact that private sector wages in the formal sector are significantly higher than in the public sector, the increase in civil service wages is not likely to affect private sector wages much. During the period 2001-04 (including the salary increase and the transportation allowance contained in the supplementary budget) real civil service wages will have declined by about 10 percent. In 2004, the civil service wage bill will
} 
reunified army and the demobilization of 50,000 soldiers (100,000 less than originally planned for 2004); and (iii) the retirement of 10,000 civil servants. Pro-poor expenditure, including infrastructure, would increase by about 5 percentage points of GDP, while security expenditure would remain unchanged. Because the DRC has obtained debt relief beyond the enhanced HIPC Initiative, external debt service has been revised downward by one percent of GDP in the supplementary budget. Overall, the domestic primary surplus, on a cash basis, is projected at 1 percent of GDP, while the overall consolidated cash deficit (including the projected net financial losses of the BCC) would widen to 3.2 percent of GDP in 2004 (Tables 4A and 4B). The decrease in net credit to the government will be about 0.6 percent of GDP instead of the programmed zero increase.

21. The 2004 revenue objective is based on a higher projected international oil price, continuation of tax administration reforms and improvements in tax compliance (MEFP, paras. 47 and 48). In particular, the authorities will (i) conduct an inventory of all exemptions and rationalize those not in conformity with the investment, mining and forestry codes; (ii) revise the existing convention between the MIBA mining company and the government to ensure tax collection from that company; (iii) adopt the legislative texts necessary for the opening of the tax office for medium-sized enterprises starting July 1 2004; and (iv) ensure the uniform implementation of all tax and customs laws throughout the country.

\section{Continued progress in public expenditure management will be key for the} effective tracking of public expenditure and transparency of fiscal management (MEFP, para. 51). Monitoring of public expenditure will be strengthened by producing monthly budget tracking statements according to the four phases of the expenditure chain on the tenth day of each following month for all types of expenditure. Other measures envisaged for 2004 include (i) the closing of the government accounts in commercial banks that do not meet the criteria established by the Treasury Directorate; (ii) following up on the calendar necessary for the establishment of a double-entry accounting framework by end 2004; (iii) the adoption of regulations governing the eligibility and management of "budgets annexes"; and (iv) the implementation of the short-term recommendations resulting from the comprehensive audit of the payroll system, conducted with the help of France. A nation-wide civil service census will start in September 2004 (MEFP, paras. 49 and 51).

reach only 1.9 percent of GDP; one of the lowest in Africa. The lowest salary grade, excluding transportation allowances that are granted only in Kinshasa and Lumumbashi, will now reach US\$2 per month, while the highest grade will reach US\$20. If the military and the police are included, the wage bill amounts to 3.5 percent of GDP. 
Comparison of the 2004 Approved Budget with the Supplementary Budget

\begin{tabular}{|c|c|c|c|c|c|c|}
\hline & IMF CR No. 04/97 & $\begin{array}{l}\text { In percent of } \\
\text { GDP }\end{array}$ & $\begin{array}{l}2004 \text { Approved } \\
\text { budget } 1 /\end{array}$ & $\begin{array}{l}\text { In percent of } \\
\text { GDP }\end{array}$ & $\begin{array}{c}2004 \\
\text { Supplementary } \\
\text { budget }\end{array}$ & $\begin{array}{l}\text { In percent of } \\
\text { GDP }\end{array}$ \\
\hline A. Total revenue & 335,238 & 13.2 & 335,238 & 13.2 & 385,866 & 15.0 \\
\hline Of which: total project grants & 63,834 & 2.5 & 63,834 & 2.5 & 121,790 & 4.7 \\
\hline Of which: total HIPC grants & 44,331 & 1.7 & 44,331 & 1.7 & 28,687 & 1.1 \\
\hline B. Total expenditure & 412,225 & 16.3 & 412,225 & 16.3 & 468,601 & 18.3 \\
\hline I. Current expenditure & 197,895 & 7.8 & 199,917 & 7.9 & 206,063 & 8.0 \\
\hline Wages & 67,655 & 2.7 & 81,475 & 3.2 & 88,828 & 3.5 \\
\hline Civil service workers (active) & 29,947 & 1.2 & 46,206 & 1.8 & 46,206 & 1.8 \\
\hline Civil service workers (retired) & 3,194 & 0.1 & 2,223 & 0.1 & 3,292 & 0.1 \\
\hline Institutions & 7,273 & 0.3 & 7,198 & 0.3 & 7,273 & 0.3 \\
\hline Army & 17,550 & 0.7 & 15,555 & 0.6 & 22,000 & 0.9 \\
\hline Police and security services & 7,691 & 0.3 & 8,293 & 0.3 & 8,057 & 0.3 \\
\hline Payments for retirement of 10,000 people & 2,000 & 0.1 & 2,000 & 0.1 & 2,000 & 0.1 \\
\hline Other current expenditure & 128,740 & 5.1 & 118,442 & 4.7 & 113,735 & 4.4 \\
\hline Institutions & 18,000 & 0.7 & 15,149 & 0.6 & 17,170 & 0.7 \\
\hline Of which: Electoral Commission & n.a. & n.a. & n.a. & n.a. & 1,474 & 0.1 \\
\hline Ministries & 30,000 & 1.2 & 29,317 & 1.2 & 30,617 & 1.2 \\
\hline Of which: integrated expenditures from "special budgets" & n.a. & n.a. & n.a. & n.a. & 1,300 & 0.1 \\
\hline Transfers to provinces and deconcentrated units & 11,657 & 0.5 & 11,657 & 0.5 & 12,432 & 0.5 \\
\hline Interest payments & 32,603 & 1.3 & 32,603 & 1.3 & 20,429 & 0.8 \\
\hline Domestic debt & 2,000 & 0.1 & 2,000 & 0.1 & 2,000 & 0.1 \\
\hline External debt (after debt relief) 2/ & 30,603 & 1.2 & 30,603 & 1.2 & 18,429 & 0.7 \\
\hline Centralized payments (utilities) & 25,885 & 1.0 & 17,621 & 0.7 & 22,361 & 0.9 \\
\hline Transfers to revenue collecting agencies & 10,595 & 0.4 & 10,595 & 0.4 & 10,725 & 0.4 \\
\hline Transfers to public agencies and "budget annexes" & 1,500 & 0.1 & 1,500 & 0.1 & 1,500 & 0.1 \\
\hline Cost related to the liquidation of private commercial banks & 0 & 0.0 & 0 & 0.0 & 2,000 & 0.1 \\
\hline II. Public debt $3 /$ & $\mathbf{2 , 0 0 0}$ & 0.1 & $\mathbf{2 , 0 0 0}$ & 0.1 & 16,149 & 0.6 \\
\hline III. HIPC spending & 44,331 & 1.7 & 44,331 & 1.7 & 44,331 & 1.7 \\
\hline IV. Capital expenditures & 151,999 & 6.0 & 149,977 & 5.9 & 186,059 & 7.3 \\
\hline Of which: DDR (financed by the World Bank) & n.a. & n.a. & n.a. & n.a. & 5,814 & 0.2 \\
\hline Of which: cost of elections (financed by external aid) & n.a. & n.a. & n.a. & n.a. & 32,958 & 1.3 \\
\hline $\mathrm{V}$. Treasury deficit of the BCC & 16,000 & 0.6 & 16,000 & 0.6 & 16,000 & 0.6 \\
\hline C. Overall balance & $-76,987$ & -3.0 & $-76,987$ & -3.0 & $-82,735$ & -3.2 \\
\hline D. Financing & 76,987 & 3.0 & 76,987 & 3.0 & 82,735 & 3.2 \\
\hline I. Net Credit to government & 0 & 0.0 & 0 & 0.0 & $-16,059$ & -0.6 \\
\hline II. Amortization of external debt (after debt relief) 2/ & $-70,259$ & -2.8 & $-70,259$ & -2.8 & $-43,673$ & -1.7 \\
\hline III. Total external disbursements & 147,246 & 5.8 & 147,246 & 5.8 & 142,467 & 5.6 \\
\hline Project & 80,465 & 3.2 & 80,465 & 3.2 & 56,568 & 2.2 \\
\hline Budget & 66,781 & 2.6 & 66,781 & 2.6 & 85,899 & 3.3 \\
\hline
\end{tabular}

1/ The 2004 budget was discussed during the Third Review (IMF Country Report No. 04/97).

2/ Includes deposits in the HIPC sub-account at the BCC.

3/ Repayment of arrears (including cross-arreas with the private sector).

\section{Progress on the decentralization agenda will depend on an evaluation, with} Fund technical assistance, of the financial impact of the draft decentralization law that was recently submitted to Parliament (Box 2). Meanwhile, the authorities have agreed that (i) the provincial budgets will be balanced; (ii) no provincial external borrowing be made without the prior approval of the Minister of Finance; (iii) no provincial domestic borrowing be allowed; (iv) revenue transfers from the central government to the provinces be maintained at their current levels; (v) central government responsibility for tax and customs administration be maintained; and (vi) no new taxes be created without the approval of the Minister of Finance. 


\section{Box 2. Territorial Organization and Fiscal Decentralization}

A key goal of the Government of National Unity is the effective reunification of the country, which was divided and governed by different factions since the beginning of the civil war in 1998. The DRC comprises eleven provinces. Until 2003, the government only controlled six of them, covering close to 60 percent of the population.

\section{Fiscal relations between central and local governments}

The territorial organization of the country provides for:

- $\quad$ Four levels of local government called Entités Administratives Décentralisées (EAD), each having a separate legal personality, its own budget, and the right to raise local taxes.

- $\quad$ Resources of EADs consist of local taxes, specified shares of the collection of certain central taxes, and transfers from the central government. Since 1998, these transfers have been officially set at 20 percent of certain tax revenues collected in the provinces, but with only 10 percent being automatically transferred.

- $\quad$ Expenditures by the central government in the provinces cover mainly wages and pensions of civil servants.

The authorities agreed to delay the adoption of the draft decentralization law by Parliament until a full assessment of its fiscal impact is completed, with the help of Fund technical assistance. In particular, this law includes: (i) greater fiscal autonomy for EADs with an increased transfer rate of 30 percent of revenues (but the expenditure responsibilities need to be defined); and (ii) the possibility for provinces to borrow externally and become shareholders of local companies.

\section{Monetary and financial sector policies}

24. Monetary policy in $\mathbf{2 0 0 4}$ will continue to aim at maintaining price stability in the context of the floating exchange rate regime. The $\mathrm{BCC}$ will face the twin challenges of meeting the demand for CGF in the whole country and improving its international reserve position. To meet these objectives, the $\mathrm{BCC}$ will pursue a more active policy to purchase foreign exchange in the market and carefully prepare the issuance of higher-denomination CGF notes, based on a study on the desired volume and composition of currency. The BCC is also enhancing its refinancing instruments to meet the needs of the economy, and will put in place a unified payments system (MEFP, paras. 54 and 55). 


\section{Broad money is projected to grow by 29 percent in 2004, reflecting a continued} fall in velocity in line with increased demand for CGF and confidence in the banking system. Credit to the government is programmed to be reduced by 14 percent of the beginning-of-period broad money stock, permitting a significant increase in the BCC's NFA position and adequate room for credit to the private sector (MEFP, para. 53).

\section{The BCC will pursue its restructuring efforts guided by its updated action plan,} with particular emphasis in the remainder of 2004 and in 2005 on (i) the preparation of its recapitalization (with support from Belgium), based on the audited accounts for 2003; (ii) the reform of its accounting system and foreign exchange operations through the introduction of new software applications; (iii) a further strengthening of banking supervision; and (iv) strict adherence to its treasury plan (MEFP, paras. 56-58). The strengthening of the banking sector and financial intermediation will continue with the implementation by banks of their restructuring plans monitored by the $\mathrm{BCC}$ and the on-going reform of the tax and regulatory framework for the sector. The liquidation of the three public banks and the six private banks will be accelerated, with particular emphasis on implementing the social plans for the three public banks, with financing from the World Bank (MEFP, paras. 16 and 59). In addition, the $\mathrm{BCC}$ is developing a plan for the reopening of its branch offices throughout the country.

\section{External financing issues.}

27. The 2004 program is financed through higher external grants than originally projected. The authorities will continue to borrow on highly concessional terms in line with the assumptions of the HIPC Decision Point Document (7/14/2003). They are committed to signing all outstanding agreements with multilateral and Paris Club creditors and seeking the participation of all creditors in the HIPC Initiative. The authorities are in the process of strengthening debt management (a completion point trigger), with the acquisition of a computerized debt-recording system. Finally, the authorities are requesting additional IMF interim assistance of SDR 1.131 million to cover 50.7 percent of PRGF interest obligations falling due during the period August 1, 2004 to July 31, 2005. Adequate financing assurances continue to be in place.

\section{B. Structural Policies}

28. On the structural side, far-reaching reforms will continue to be implemented with the help of development partners, primarily the World Bank (MEFP, paras. 60-75). The effective restructuring or privatization of public enterprises has been initiated in 2004, starting with those sectors that have a direct impact on production costs - electricity, water, transportation, and communications. Negotiations to settle the government's cross-arrears with the domestic private sector, to be financed by the World Bank, will start shortly (MEFP, para. 26).

Governance will continue to be strengthened, and the authorities have decided to conduct an audit of the diamond sector, with EU assistance (MEFP, para. 66). 


\section{Poverty Reduction}

29. The action plan to draw up the full poverty reduction strategy paper (PRSP) has been modified to ensure its completion, with the help of external partners, by the established deadline of August 2005 (MEFP, para. 76). The participatory consultations and the quantitative surveys, needed to determine the actual extent and nature of poverty, are in an advanced state of preparation, and are scheduled to take place in the June-December 2004 period. In their joint assessment, ${ }^{8}$ the staffs of the Fund and the World Bank consider the progress made by the authorities in drawing up their full PRSP to be satisfactory.

\section{Administrative Capacity Building, Technical Assistance, and Statistical Issues}

30. The DRC's administrative and statistical capacities have been improving, with technical assistance from the international community. However, weaknesses remain, especially in the areas of national accounts and the balance of payments. Following the recommendations of a STA multitopic mission in October 2003, a Fund resident expert is expected to be stationed in Kinshasa shortly (MEFP, para. 78). The DRC has begun participating in the General Data Dissemination System (GDDS), showing the authorities' commitment to improve statistics. Technical assistance is being provided, particularly to buttress administrative capacity, including at the provincial level.

\section{PRGF ARrangement Monitoring}

31. Program implementation in 2004 will continue to be monitored by means of semiannual reviews, semiannual quantitative and structural performance criteria (March and September 2004), and quarterly benchmarks (June, September, and December 2004). To ensure the success of the program, the government will implement before end-June 2004 three prior actions (MEFP, paras. 79-82, and Tables 4 and 5).

\section{StafF Appraisal}

\section{The staff commends the government for its efforts to achieve effective}

reunification, notably the appointment of provincial governors, the preparation of the national DDR program that would cut the size of the army by about half, and the drawing up of a tentative calendar for the local, legislative, and presidential elections in the second half of 2005 .

33. Demobilization and the holding of elections, together with the rehabilitation of administrative capacity at the provincial level and the adoption and implementation of

\footnotetext{
${ }^{8}$ www.imf.org.
} 
a decentralization law, represent major challenges to the authorities, and the staff welcomes the intention of the international community to assist them in their efforts. In this connection, the staff reiterates the importance of timely and well-coordinated external assistance covering the entire territory, including in the form of grants and direct budgetary assistance.

\section{Overall, the PRGF-supported program has been satisfactorily implemented so} far, with good progress in the structural area despite some delays. This performance has permitted the country to move from the stabilization phase to the reconstruction phase, as envisaged in its I-PRSP. In the period leading up to the elections in the second half of 2005, the authorities will need to maintain good coordination of economic and financial policies to preserve macroeconomic stability, maintain the momentum of their reform agenda, and combat poverty. In this connection, the staff notes the authorities' intention to complete a full PRSP by the originally targeted date of August 2005.

35. The staff endorses the authorities' overall policy priorities for 2004, namely, the implementation of effective decentralization that preserves fiscal control, the demobilization of 50,000 soldiers, keeping security spending constant in terms of GDP while boosting propoor spending, building up foreign reserves and, in the structural area, the adoption and implementation of the laws to combat money laundering and corruption, the restructuring of public enterprises and the banking system, and the settlement of the government's net crossarrears with the private sector.

\section{Fiscal policy in $\mathbf{2 0 0 4}$ should aim at further consolidation and redirecting} expenditure toward pro-poor spending. The new budget classification, introduced in 2003, together with the full implementation of the new expenditure procedures, the preparation of budget tracking statements, and the continued adherence to a monthly cash plan, should allow for effective tracking of expenditure. On the revenue side, the staff encourages the authorities to continue implementing their comprehensive tax reform, redeploy the revenue-collecting agencies throughout the country, improve tax compliance, rationalize existing exemptions and refrain from granting ad hoc ones.

37. The BCC should continue to aim at achieving the overriding objective of price stability within the framework of the floating exchange rate system, a policy that continues to serve the country well. The staff encourages the monetary authorities to continue to strengthen their monetary policy instruments to enable them, inter alia, to build up foreign reserves to a comfortable level.

38. The staff commends the BCC for the progress in implementing its action plan to improve its accounting, operations, and management. The $\mathrm{BCC}$ should continue to steadfastly implement this plan as well as its monthly cash-flow plan. The BCC is encouraged to implement the necessary measures to remove the qualification issued by the external auditor on the annual audited financial statements, and continue its efforts to fully 
implement International Financial Reporting Standards (IFRS) and periodically reconcile the IMF accounts.

39. The staff considers that the authorities are engaged in good-faith negotiations with external private creditors with which the DRC has outstanding arrears and that relations with those creditors have not undermined so far program implementation.

40. The authorities' intention to deepen the structural reforms is welcome, in particular the promotion of good governance, and the fight against corruption and money laundering. The staff encourages the authorities to rigorously implement the reform of the public enterprises and the restructuring of the banking system. At the same time, the reforms to strengthen the legal system and the judiciary should be firmly pursued.

41. The staff notes that the unsettled security situation in the east of the country continues to represent a risk to the program, particularly to the achievement of fiscal targets, the financing of foreign-financed projects, and the reduction in poverty. The forthcoming Regional Peace Conference for the Great Lakes Region could be an important avenue to stabilize the situation.

42. The staff recommends completion of the fourth review under the PRGF arrangement and supports the authorities' request for waivers for the nonobservance of three quantitative performance criteria. The staff notes the authorities' overall track record in bringing about peace and implementing economic and financial reforms, as well as the strength of their program for 2004 and the medium term, particularly given the measures taken to strengthen fiscal policy and enhance monetary policy instruments. The staff also notes that strong corrective action has been taken to address the slippages of 2003 and that in the absence of short delays in external disbursements the performance criterion on net credit to the government would have been met while the nonobservance of the criteria on net foreign assets and net domestic assets would have been minimal.

43. The staff also recommends that the authorities' request for an additional tranche of IMF interim assistance under the enhanced HIPC Initiative be approved. 
Figure 3. Democratic Republic of the Congo: Exchange Rate Indices, January 1996-March 2004 $($ Index $1990=100)$

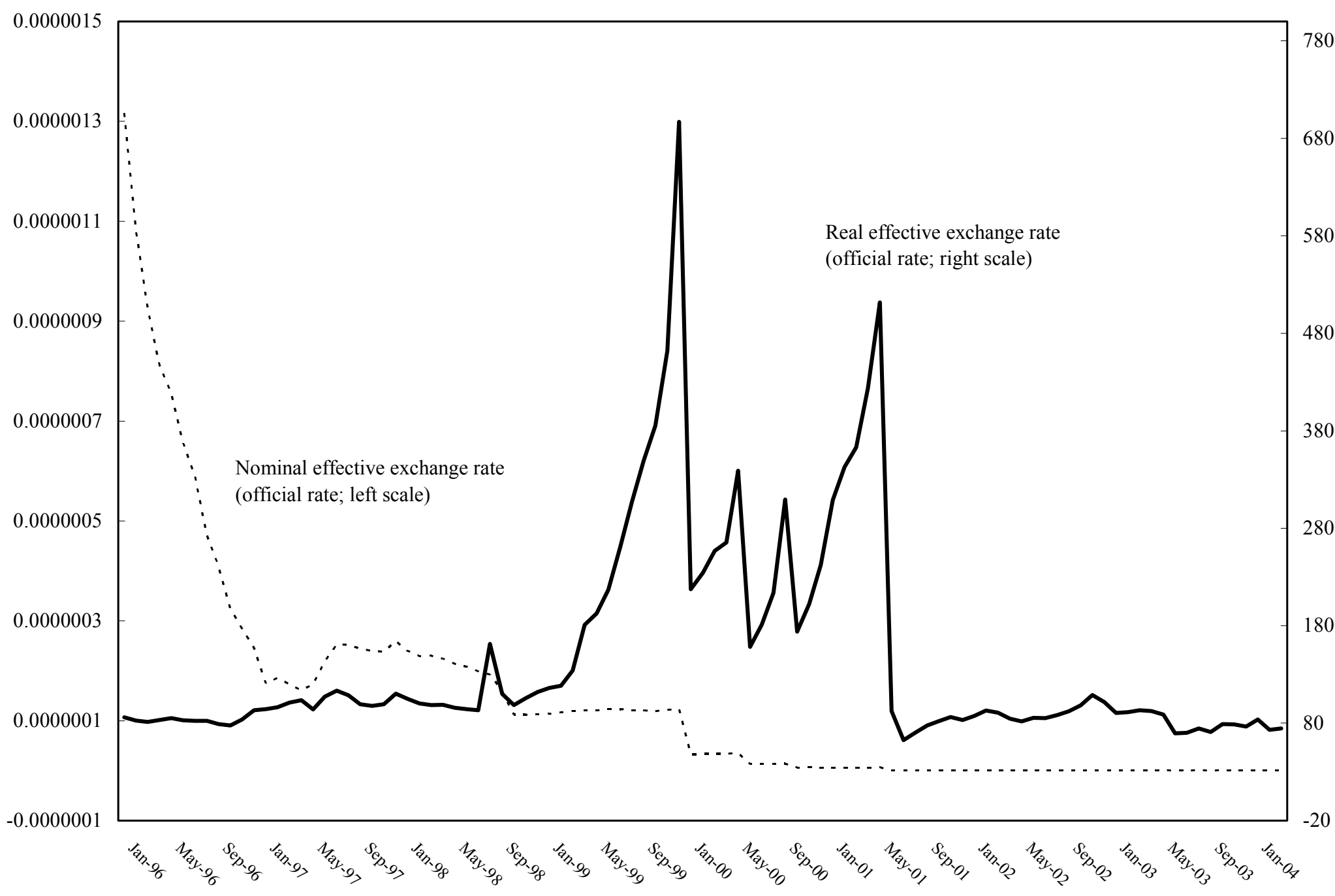

Sources: IMF, Information Notice System (INS). 
Table 1. Democratic Republic of the Congo: Fund Position During the PRGF Arrangement, 2002-05

\begin{tabular}{|c|c|c|c|c|c|c|c|c|}
\hline & \multicolumn{2}{|c|}{2002} & \multicolumn{2}{|c|}{2003} & \multicolumn{2}{|c|}{2004} & \multicolumn{2}{|c|}{2005} \\
\hline & Jan.-Jun. & $\overline{\text { Jul.-Dec. }}$ & Jan.-Jun. & Jul.-Dec. & Jan.-Jun. & Jul.-Dec. & Jan.-Jun. & $\overline{\text { Jul.-Dec. }}$ \\
\hline & \multicolumn{8}{|c|}{ (In millions of SDRs) } \\
\hline \multicolumn{9}{|l|}{ Disbursements } \\
\hline Poverty Reduction and Growth Facility (PRGF) & 420.0 & 0.0 & 26.7 & 26.7 & 26.7 & 26.7 & 26.7 & 26.7 \\
\hline Repurchases/repayments & 0.0 & 0.0 & 0.0 & 0.0 & 0.0 & 0.0 & 0.0 & 0.0 \\
\hline Ordinary resources & 0.0 & 0.0 & 0.0 & 0.0 & 0.0 & 0.0 & 0.0 & 0.0 \\
\hline PRGF & 0.0 & 0.0 & 0.0 & 0.0 & 0.0 & 0.0 & 0.0 & 0.0 \\
\hline Arrears clearance (on 6/12/02) & 403.9 & 0.0 & 0.0 & 0.0 & 0.0 & 0.0 & 0.0 & 0.0 \\
\hline Charges and interest & 0.0 & 1.9 & 1.7 & 1.7 & 1.8 & 1.8 & 1.9 & 1.9 \\
\hline Ordinary resources & 0.0 & 0.0 & 0.0 & 0.0 & 0.0 & 0.0 & 0.0 & 0.0 \\
\hline PRGF & 0.0 & 1.1 & 1.1 & 1.1 & 1.2 & 1.2 & 1.3 & 1.3 \\
\hline SDR charges & 0.0 & 0.8 & 0.6 & 0.6 & 0.6 & 0.6 & 0.6 & 0.6 \\
\hline Total Fund credit outstanding (end of period) & 420.0 & 420.0 & 446.7 & 473.3 & 500.1 & 526.8 & 553.4 & 580.1 \\
\hline Ordinary resources & 0.0 & 0.0 & 0.0 & 0.0 & 0.0 & 0.0 & 0.0 & 0.0 \\
\hline Structural Adjustment Facility (SAF) arrangements & 0.0 & 0.0 & 0.0 & 0.0 & 0.0 & 0.0 & 0.0 & 0.0 \\
\hline Charges/interest & 0.0 & 0.0 & 0.0 & 0.0 & 0.0 & 0.0 & 0.0 & 0.0 \\
\hline \multirow[t]{2}{*}{ PRGF } & 420.0 & 420.0 & 446.7 & 473.3 & 500.1 & 526.6 & 553.3 & 580.0 \\
\hline & \multicolumn{8}{|c|}{ (In percent of quota, unless otherwise specified) } \\
\hline Total Fund credit outstanding (end of period) & 78.8 & 78.8 & 83.8 & 88.8 & 93.8 & 98.8 & 103.8 & 108.8 \\
\hline PRGF disbursements & 78.8 & 0.0 & 5.0 & 5.0 & 5.0 & 5.0 & 5.0 & 5.0 \\
\hline Repurchases/repayments & 0.0 & 0.0 & 0.0 & 0.0 & 0.0 & 0.0 & 0.0 & 0.0 \\
\hline Charges and interest & 0.0 & 0.4 & 0.3 & 0.3 & 0.3 & 0.3 & 0.4 & 0.4 \\
\hline Debt service (in percent of exports of goods and services) $1 /$ & & 0.2 & & 0.2 & & 0.2 & & 0.2 \\
\hline
\end{tabular}

Sources: International Monetary Fund, Finance Department; and IMF staff projections.

1/ After normalization, new financing and debt relief (including HIPC). Ratio for the entire year. 
Table 2. Democratic Republic of the Congo: Proposed Schedule of Disbursements Under the PRGF Arrangement, 2002-05

Amount $\quad$ Availability Date Conditions Necessary for Disbursement 1/

SDR 420 million

SDR 26.7 million

SDR 26.7 million

SDR 26.7 million

SDR 26.7 million

SDR 26.7 million

SDR 26.7 million
June 12, 2002

April 8, 2003

July 23, 2003

March 3, 2004

July 12, 2004

January 15,2005

May 30, 2005
Following Executive Board Approval of the three-year Poverty Reduction and Growth Facility arrangement.

Observance of the performance criteria for September 30, 2002 and completion of the first review under the PRGF arrangement.

Observance of the performance criteria for March 31, 2003 and completion of the second review under the PRGF arrangement.

Observance of the performance criteria for September 30, 2003 and completion of the third review under the PRGF arrangement.

Observance of the performance criteria for March 31, 2004 and completion of the fourth review under the PRGF arrangement.

Observance of the performance criteria for September 30, 2004 and completion of the fifth review under the PRGF arrangement.

Observance of the performance criteria for March 31, 2005 and completion of the sixth review under the PRGF arrangement.

Source: International Monetary Fund.

1/ Other than the generally applicable conditions under the Poverty Reduction and Growth Facility (PRGF) arrangement. 


\begin{tabular}{|c|c|c|c|c|c|c|c|c|c|c|}
\hline & \multirow[t]{2}{*}{2002} & \multicolumn{2}{|c|}{2003} & \multicolumn{2}{|c|}{2004} & \multicolumn{2}{|c|}{2005} & \multicolumn{2}{|c|}{2006} & \multirow{2}{*}{$\begin{array}{l}2007 \\
\text { Proj. }\end{array}$} \\
\hline & & $\begin{array}{r}\text { Country } \\
\text { Report } \\
\text { No. 04/97 }\end{array}$ & Est. & $\begin{array}{r}\text { Country } \\
\text { Report } \\
\text { No. } 04 / 97\end{array}$ & Rev. Prog. & $\begin{array}{c}\text { Country } \\
\text { Report } \\
\text { No. } 04 / 97\end{array}$ & Proj. & $\begin{array}{c}\text { Country } \\
\text { Report } \\
\text { No. } 04 / 97\end{array}$ & Proj. & \\
\hline & \multicolumn{10}{|c|}{ (Annual percentage changes) } \\
\hline \multicolumn{11}{|l|}{ Output and prices } \\
\hline Real GDP & 3.5 & 5.0 & 5.6 & 6.0 & 6.3 & 7.0 & 7.0 & 7.0 & 7.0 & 7.0 \\
\hline GDP deflator & 32.0 & 12.5 & 12.4 & 5.4 & 5.0 & 5.0 & 5.0 & 4.5 & 5.0 & 5.0 \\
\hline Consumer prices, annual average & 25.3 & 13.0 & 12.8 & 6.0 & 5.0 & 5.5 & 5.0 & 5.0 & 5.0 & 5.0 \\
\hline Consumer prices, end-of-period & 15.8 & 4.4 & 4.4 & 6.0 & 6.0 & 5.0 & 5.0 & 5.0 & 5.0 & 5.0 \\
\hline \multicolumn{11}{|l|}{ External sector } \\
\hline Exports, f.o.b. (in U.S. dollars) & 22.2 & 15.5 & 19.1 & 10.7 & 10.3 & 11.5 & 5.1 & 11.2 & 8.7 & 9.5 \\
\hline Imports, f.o.b. (in U.S. dollars) & 35.4 & 25.4 & 28.5 & 26.8 & 33.4 & 15.1 & 20.9 & 11.2 & -2.2 & 9.8 \\
\hline Export volume & 21.9 & 11.5 & 13.6 & 11.7 & 4.3 & 12.5 & 7.0 & 12.2 & 11.5 & 10.5 \\
\hline Import volume & 30.5 & 18.9 & 19.6 & 24.7 & 23.5 & 13.8 & 22.4 & 10.0 & 1.0 & 10.0 \\
\hline Terms of trade & 3.9 & -1.7 & -2.4 & -2.5 & -2.1 & -2.0 & -0.6 & -2.0 & 0.6 & -0.7 \\
\hline Nominal effective exchange rate $1 /$ & -71.1 & $\ldots$ & -19.5 & $\ldots$ & $\ldots$ & $\ldots$ & $\ldots$ & $\ldots$ & $\ldots$ & $\ldots$ \\
\hline Real effective exchange rate $1 /$ & -56.2 & $\ldots$ & -11.1 & $\ldots$ & $\ldots$ & $\ldots$ & $\ldots$ & $\ldots$ & $\ldots$ & $\ldots$ \\
\hline & \multicolumn{10}{|c|}{ (Annual change in percent of beginning-of-period broad money; unless otherwise indicated) } \\
\hline \multicolumn{11}{|c|}{ (2t) } \\
\hline Broad money & 25.7 & 31.2 & 32.7 & 25.0 & 28.7 & $\ldots$ & $\ldots$ & & $\ldots$ & $\ldots$ \\
\hline Net foreign assets & 10.4 & 10.9 & -14.1 & 22.8 & 35.1 & $\ldots$ & $\ldots$ & $\ldots$ & $\ldots$ & $\ldots$ \\
\hline Net domestic credit & -13.3 & 18.4 & 39.0 & 5.9 & -1.1 & $\ldots$ & $\ldots$ & $\ldots$ & $\ldots$ & $\ldots$ \\
\hline Net credit to the government & -14.2 & 10.9 & 32.1 & 0.0 & -13.8 & $\ldots$ & $\ldots$ & $\ldots$ & $\ldots$ & $\ldots$ \\
\hline Credit to the private sector & 0.6 & 8.0 & 7.5 & 5.7 & 11.3 & $\ldots$ & $\ldots$ & $\ldots$ & $\ldots$ & $\ldots$ \\
\hline Credit to the parastatals & 0.4 & -0.4 & -0.6 & 0.2 & 1.4 & $\ldots$ & $\ldots$ & $\ldots$ & $\ldots$ & $\ldots$ \\
\hline Central bank refinance rate (level in percent) & 24 & 15 & 8 & $\ldots$ & $\ldots$ & $\ldots$ & $\ldots$ & $\ldots$ & $\ldots$ & $\ldots$ \\
\hline & \multicolumn{10}{|c|}{ (In percent of GDP) } \\
\hline \multicolumn{11}{|l|}{ Central government finances } \\
\hline Revenue (excluding grants) & 7.9 & 8.2 & 7.7 & 9.0 & 9.2 & 10.1 & 10.3 & 11.0 & 11.0 & 11.5 \\
\hline Grants (excluding humanitarian aid) & 0.4 & 1.9 & 2.0 & 4.3 & 5.9 & 6.8 & 10.0 & 8.8 & 8.2 & 8.0 \\
\hline Expenditure 2/ & 10.3 & 13.2 & 13.6 & 17.6 & 19.3 & 20.2 & 25.3 & 21.5 & 21.6 & 21.4 \\
\hline Domestic primary cash balance 3 / & 1.4 & 0.5 & -0.2 & 2.1 & 1.0 & 3.3 & 2.3 & 4.7 & 3.6 & 4.0 \\
\hline Overall balance (commitment basis) & -2.0 & -3.1 & -3.9 & -4.3 & -4.2 & -3.3 & -5.0 & -1.7 & -2.4 & -1.8 \\
\hline Overall consolidated cash balance 4/ & -0.1 & -1.4 & -2.0 & -3.0 & -3.2 & -1.5 & -3.2 & 0.3 & -0.5 & -0.2 \\
\hline \multicolumn{11}{|l|}{ Investment and saving } \\
\hline Gross national savings & 11.2 & 11.1 & 13.4 & 13.3 & 16.6 & 15.8 & 21.1 & 18.9 & 20.3 & 20.4 \\
\hline Government & 1.3 & -0.2 & -0.9 & 2.7 & 4.1 & 6.3 & 8.7 & 9.8 & 8.4 & 8.5 \\
\hline Nongovernment & 9.9 & 11.2 & 14.3 & 10.6 & 12.5 & 9.5 & 12.3 & 9.1 & 11.9 & 11.9 \\
\hline Gross domestic savings & 4.0 & 5.2 & 5.7 & 5.9 & 6.5 & 7.4 & 7.4 & 9.0 & 9.0 & 9.1 \\
\hline Government & 1.1 & 1.0 & 0.1 & 1.5 & 1.0 & 2.7 & 1.8 & 4.1 & 3.0 & 3.0 \\
\hline Nongovernment & 3.0 & 4.2 & 5.6 & 4.5 & 5.5 & 4.7 & 5.7 & 4.9 & 5.9 & 6.1 \\
\hline Investment & 9.0 & 12.1 & 12.2 & 17.4 & 18.6 & 20.6 & 24.7 & 22.5 & 21.8 & 21.5 \\
\hline Government $5 /$ & 1.0 & 2.6 & 2.7 & 6.9 & 8.1 & 9.6 & 13.7 & 11.5 & 10.8 & 10.3 \\
\hline Nongovernment 6/ & 8.0 & 9.5 & 9.5 & 10.5 & 10.5 & 11.0 & 11.0 & 11.0 & 11.0 & 11.2 \\
\hline
\end{tabular}




\begin{tabular}{|c|c|c|c|c|c|c|c|c|c|c|}
\hline & \multirow[t]{2}{*}{2002} & \multicolumn{2}{|c|}{2003} & \multicolumn{2}{|c|}{2004} & \multicolumn{2}{|c|}{2005} & \multicolumn{2}{|c|}{2006} & \multirow{2}{*}{2007} \\
\hline & & $\begin{array}{c}\text { Country } \\
\text { Report } \\
\text { No. } 04 / 97\end{array}$ & Est. & $\begin{array}{c}\text { Country } \\
\text { Report } \\
\text { No. 04/97 }\end{array}$ & Rev. Prog. & $\begin{array}{c}\text { Country } \\
\text { Report } \\
\text { No. 04/97 }\end{array}$ & Proj. & $\begin{array}{c}\text { Country } \\
\text { Report } \\
\text { No. } 04 / 97\end{array}$ & Proj. & \\
\hline & \multirow{2}{*}{\multicolumn{10}{|c|}{ (In millions of U.S. dollars; unless otherwise indicated) }} \\
\hline Balance of payments & & & & & & & & & & \\
\hline Exports of goods and nonfactor services & $1,174.2$ & $1,383.9$ & $1,428.9$ & $1,529.9$ & $1,588.7$ & $1,697.9$ & $1,680.9$ & $1,880.5$ & $1,815.9$ & $1,979.0$ \\
\hline (in percent of GDP) & 21.2 & 24.8 & 25.2 & 24.9 & 23.5 & 25.5 & 22.9 & 26.0 & 22.7 & 22.7 \\
\hline Imports of goods and nonfactor services & $1,446.5$ & $1,771.8$ & $1,801.4$ & $2,231.6$ & $2,410.0$ & $2,574.2$ & $2,946.3$ & $2,858.4$ & $2,839.8$ & $3,059.9$ \\
\hline (in percent of GDP) & 26.1 & 31.7 & 31.7 & 36.3 & 35.7 & 38.6 & 40.2 & 39.5 & 35.5 & 35.1 \\
\hline \multicolumn{11}{|l|}{ External current account, including grants, } \\
\hline before debt relief (in percent of GDP) & -2.8 & -2.0 & 0.6 & -6.0 & -3.3 & -7.6 & -6.5 & -7.6 & -5.4 & -5.3 \\
\hline $\begin{array}{l}\text { External current account, excluding grants, } \\
\text { before debt relief (in percent of GDP) }\end{array}$ & -10.3 & -10.7 & -8.1 & -14.7 & -13.3 & -16.8 & -18.7 & -16.7 & -13.7 & -12.0 \\
\hline $\begin{array}{l}\text { External current account, including grants, } \\
\text { after debt relief (in percent of GDP) } 7 /\end{array}$ & 2.2 & -1.1 & 1.2 & -4.1 & -2.0 & -4.7 & -3.6 & -3.5 & -1.5 & -1.2 \\
\hline Gross official reserves (end-of-period) & 74.7 & 156.6 & 96.6 & 272.1 & 209.3 & 382.2 & 318.2 & 479.1 & 479.1 & 611.9 \\
\hline $\begin{array}{l}\text { Gross official reserves (in weeks of non-aid-relate } \\
\text { imports of goods and nonfactor services) }\end{array}$ & 2.9 & 5.3 & 3.3 & 8.2 & 6.2 & 10.7 & 8.9 & 12.1 & 11.4 & 14.1 \\
\hline \multicolumn{11}{|l|}{ External public debt } \\
\hline Total stock, including IMF 8/ & 10,659 & 10,627 & 10,563 & 10,714 & 10,783 & 10,970 & 11,032 & 5,687 & 5,512 & 5,744 \\
\hline Net present value of debt (NPV) $9 /$ & 7,933 & 7,376 & 7,274 & 7,538 & 7,546 & 7,837 & 7,756 & 2,095 & 1,859 & 2,009 \\
\hline $\begin{array}{l}\text { Scheduled debt service } 7 / \\
\text { In percent of exports of goods and }\end{array}$ & 34.2 & 139.9 & 126.7 & 140.3 & 91.7 & 204.9 & 135.0 & 313.5 & 198.7 & 226.7 \\
\hline nonfactor services & 2.9 & 10.1 & 8.9 & 9.2 & 5.8 & 12.1 & 8.0 & 16.7 & 10.9 & 11.5 \\
\hline In percent of government revenue, excl. grants & 7.4 & 24.8 & 23.0 & 17.3 & 9.0 & 18.2 & 9.1 & 21.9 & 12.9 & 13.3 \\
\hline \multicolumn{11}{|l|}{ Memorandum items: } \\
\hline \multicolumn{11}{|l|}{ Exchange rate } \\
\hline $\begin{array}{l}\text { Units of local currency per U.S. dollar } \\
\text { (end-of-period) }\end{array}$ & 382.0 & 372.5 & 372.5 & 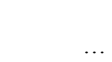 & & & $\ldots$ & & & $\ldots$ \\
\hline Nominal GDP (in billions of Congo francs) & 1,922 & 2,270 & 2,299 & 2,537 & 2,565 & 2,851 & 2,881 & 3,189 & 3,237 & 3,637 \\
\hline (Percentage changes) & 36.6 & 18.5 & 19.6 & 11.7 & 11.6 & 12.4 & 12.4 & 11.9 & 12.4 & 12.4 \\
\hline
\end{tabular}

Sources: Congolese authorities; and IMF staff estimates and projections.

$1 /$ Change in annual average. Minus sign indicates depreciation.

2/ Includes interest due on external debt (including debt service on rescheduling) and, from 2003 onward, expenditure financed by resources released under the enhanced HIPC Initiative.

3/ Revenue (excluding grants) minus expenditure (excluding interest on debt, foreign-financed expenditure, and HIPC-related expenditure).

4/ Cash balance after interest rescheduling (including HIPC).

5/ From 2003 onward, includes investment financed by resources released under the enhanced HIPC Initiative.

6/ From 2003 onward, includes capital projects financed by NGOs.

7/ From 2003, after debt relief from bilateral creditors and HIPC Initiative assistance.

8/ End-of-period debt stock, including arrears and after HIPC Initiative assistance.

9/ Estimates and projections based on end-2002 DSA and after HIPC Initiative assistance. 
Table 4A. Democratic Republic of the Congo: Summary of Central Government Financial Operations, 2002-07

\begin{tabular}{|c|c|c|c|c|c|c|c|c|c|c|}
\hline & \multirow[t]{2}{*}{2002} & \multicolumn{2}{|c|}{2003} & \multicolumn{2}{|c|}{2004} & \multicolumn{2}{|c|}{2005} & \multicolumn{2}{|c|}{2006} & \multirow{2}{*}{$\begin{array}{l}2007 \\
\text { Proj. }\end{array}$} \\
\hline & & $\begin{array}{c}\text { Country } \\
\text { Report } \\
\text { No. } 04 / 97\end{array}$ & Est. & $\begin{array}{r}\text { Country } \\
\text { Report } \\
\text { No. 04/97 }\end{array}$ & Rev. Prog. & $\begin{array}{r}\text { Country } \\
\text { Report } \\
\text { No. } 04 / 97\end{array}$ & Proj. & $\begin{array}{r}\text { Country } \\
\text { Report } \\
\text { No. 04/97 }\end{array}$ & Proj. & \\
\hline & \multicolumn{10}{|c|}{ (In millions of Congo francs; unless otherwise indicated) } \\
\hline Total revenue and grants & 159,640 & 229,248 & 223,323 & 335,238 & 385,866 & 481,184 & 583,159 & 631,699 & 624,152 & 710,543 \\
\hline Total revenue & 152,193 & 185,857 & 176,817 & 227,073 & 235,389 & 286,656 & 295,944 & 349,518 & 357,200 & 419,669 \\
\hline Customs and excises (OFIDA) & 54,668 & 76,786 & 72,500 & 108,602 & 108,602 & 137,629 & 137,594 & 164,327 & 164,342 & 205,842 \\
\hline Direct and indirect taxes (DGC) & 40,505 & 51,069 & 49,038 & 63,000 & 63,000 & 86,877 & 87,017 & 116,458 & 117,304 & 132,964 \\
\hline Petroleum (royalties and taxes) & 31,507 & 36,709 & 36,386 & 30,319 & 36,907 & 30,495 & 36,822 & 29,741 & 34,766 & 33,244 \\
\hline Off-budget revenue & 11,679 & 2,100 & 2,100 & 0 & 0 & 0 & 0 & 0 & 0 & 0 \\
\hline Other & 13,834 & 19,193 & 16,793 & 25,153 & 26,880 & 31,656 & 34,511 & 38,991 & 40,788 & 47,619 \\
\hline Total grants & 7,447 & 43,391 & 46,506 & 108,165 & 150,477 & 194,528 & 287,216 & 282,181 & 266,952 & 290,874 \\
\hline Of which: HIPC assistance 1/ & $\ldots$ & 13,642 & 6,505 & 44,331 & 28,687 & 79,201 & 79,203 & 129,029 & 128,543 & 151,171 \\
\hline Total expenditure & 198,406 & 298,994 & 312,270 & 445,478 & 494,485 & 576,469 & 728,104 & 684,808 & 700,262 & 777,652 \\
\hline Current expenditure & 168,243 & 238,297 & 248,324 & 249,148 & 264,095 & 276,436 & 306,581 & 287,734 & 319,054 & 349,441 \\
\hline Wages & 39,287 & 51,510 & 57,104 & 67,655 & 88,828 & 74,261 & 110,674 & 72,657 & 111,152 & 129,155 \\
\hline Interest due $2 /$ & 61,157 & 77,891 & 79,076 & 83,856 & 78,461 & 97,084 & 90,133 & 102,140 & 93,994 & 94,066 \\
\hline Transfers and subsidies $3 /$ & 6,674 & 20,006 & 19,867 & 12,095 & 14,225 & 15,234 & 17,572 & 18,923 & 21,210 & 24,854 \\
\hline Other current expenditure & 61,125 & 88,890 & 92,277 & 85,542 & 82,581 & 89,857 & 88,203 & 94,013 & 92,698 & 101,366 \\
\hline Of which: centralized payments $4 /$ & 15,439 & 24,094 & 23,564 & 25,885 & 22,361 & 27,186 & 23,479 & 28,421 & 24,653 & 26,343 \\
\hline Off-budget expenditure & 11,679 & 2,100 & 2,100 & 0 & 0 & 0 & 0 & 0 & 0 & 0 \\
\hline Capital expenditure & 10,385 & 58,508 & 61,756 & 151,999 & 186,059 & 244,592 & 367,726 & 334,108 & 318,101 & 322,517 \\
\hline Foreign-financed & 6,657 & 43,331 & 47,154 & 144,299 & 178,359 & 235,432 & 358,566 & 323,347 & 307,340 & 310,357 \\
\hline Domestic-financed & 3,728 & 15,177 & 14,603 & 7,700 & 7,700 & 9,160 & 9,160 & 10,761 & 10,761 & 12,160 \\
\hline Other operations & 112 & 89 & 89 & 0 & 0 & 0 & 0 & 0 & 0 & 0 \\
\hline Net lending & 7,987 & 0 & 0 & 0 & 0 & 0 & 0 & 0 & 0 & 0 \\
\hline HIPC-related expenditure & $\ldots$ & 0 & 0 & 44,331 & 44,331 & 55,441 & 53,797 & 62,966 & 63,107 & 105,694 \\
\hline Overall balance (commitment basis) & $-38,766$ & $-69,746$ & $-88,947$ & $-110,240$ & $-108,618$ & $-95,285$ & $-144,945$ & $-53,109$ & $-76,109$ & $-67,110$ \\
\hline Domestic primary balance (commitment basis) $5 /$ & 21,601 & 8,085 & $-9,222$ & 54,081 & 42,054 & 98,144 & 70,335 & 153,163 & 121,379 & 152,133 \\
\hline Change in arrears $6 /$ & 4,712 & 4,109 & 4,176 & $-2,000$ & $-16,149$ & $-4,000$ & $-4,000$ & $-4,000$ & $-4,000$ & $-6,000$ \\
\hline Overall balance (cash basis, before interest rescheduling) & $-34,054$ & $-65,637$ & $-84,771$ & $-112,240$ & $-124,767$ & $-99,285$ & $-148,945$ & $-57,109$ & $-80,109$ & $-73,110$ \\
\hline Domestic primary balance (cash basis) $5 /$ & 26,313 & 12,194 & $-5,046$ & 52,081 & 25,906 & 94,144 & 66,335 & 149,163 & 117,379 & 146,133 \\
\hline Central bank operational result & $-12,719$ & $-15,000$ & $-18,430$ & $-16,000$ & $-16,000$ & $-15,000$ & $-15,000$ & $-14,000$ & $-14,000$ & $-14,000$ \\
\hline Overall consolidated balance (cash basis, & & & & & & & & & & \\
\hline before interest rescheduling) & $-46,773$ & $-80,637$ & $-103,201$ & $-128,240$ & $-140,767$ & $-114,285$ & $-163,945$ & $-71,109$ & $-94,109$ & $-87,110$ \\
\hline
\end{tabular}


Table 4A. Democratic Republic of the Congo: Summary of Central Government Financial Operations, 2002-07

\begin{tabular}{|c|c|c|c|c|c|c|c|c|c|c|}
\hline & \multirow[t]{2}{*}{2002} & \multicolumn{2}{|c|}{2003} & \multicolumn{2}{|c|}{2004} & \multicolumn{2}{|c|}{2005} & \multicolumn{2}{|c|}{2006} & \multirow{2}{*}{$\begin{array}{l}2007 \\
\text { Proj. }\end{array}$} \\
\hline & & $\begin{array}{c}\text { Country } \\
\text { Report } \\
\text { No. } 04 / 97\end{array}$ & Est. & $\begin{array}{r}\text { Country } \\
\text { Report } \\
\text { No. 04/97 }\end{array}$ & Rev. Prog. & $\begin{array}{c}\text { Country } \\
\text { Report } \\
\text { No. 04/97 }\end{array}$ & Proj. & $\begin{array}{r}\text { Country } \\
\text { Report } \\
\text { No. 04/97 }\end{array}$ & Proj. & \\
\hline & \multicolumn{10}{|c|}{ (In millions of Congo francs; unless otherwise indicated) } \\
\hline Total financing & 51,651 & 77,928 & 104,064 & 128,240 & 140,767 & 114,285 & 163,945 & 71,109 & 94,109 & 87,110 \\
\hline Domestic financing & $-17,240$ & 9,200 & 27,751 & 0 & $-16,059$ & 0 & 0 & 0 & 0 & 0 \\
\hline Banks & $-9,913$ & 9,544 & 28,095 & 0 & $-16,059$ & 0 & 0 & 0 & 0 & 0 \\
\hline Central bank & $-9,913$ & 9,544 & 28,095 & 0 & $-16,059$ & 0 & 0 & 0 & 0 & 0 \\
\hline Commercial banks & 0 & 0 & 0 & 0 & 0 & 0 & 0 & 0 & 0 & 0 \\
\hline Non bank (certificates of deposit) & $-7,327$ & -344 & -344 & 0 & 0 & 0 & 0 & 0 & 0 & 0 \\
\hline Foreign financing & 68,891 & 68,728 & 76,313 & 128,240 & 156,826 & 114,284 & 163,944 & 71,109 & 94,109 & 87,110 \\
\hline Non domestic non resident banks & 7,096 & 0 & 0 & 0 & 0 & 0 & 0 & 0 & 0 & 0 \\
\hline Amortization due before debt relief & $-91,165$ & $-95,487$ & $-94,310$ & $-82,689$ & $-77,311$ & $-126,941$ & $-115,404$ & $-198,158$ & $-181,547$ & $-190,963$ \\
\hline Change in arrears $7 /$ & $-3,599,475$ & 0 & 0 & 0 & 0 & 0 & 0 & 0 & 0 & 0 \\
\hline Additional financing & 24,663 & 60,897 & 55,673 & 147,246 & 142,467 & 184,213 & 206,471 & 236,247 & 209,434 & 212,372 \\
\hline Debt relief before HIPC assistance 8/ & 128,297 & 103,318 & 114,950 & 63,684 & 91,670 & 57,012 & 72,878 & 33,020 & 66,223 & 65,700 \\
\hline Financing gap after debt relief & 0 & 0 & 0 & 0 & 0 & 0 & 0 & 0 & 0 & 0 \\
\hline \multirow[t]{2}{*}{ Discrepancy $9 /$} & 4,878 & $-2,709$ & 863 & 0 & 0 & 0 & 0 & 0 & 0 & 0 \\
\hline & \multicolumn{10}{|c|}{ (In percent of GDP; unless otherwise indicated) } \\
\hline \multicolumn{11}{|l|}{ Memorandum items: } \\
\hline GDP (in billion of CGF) & 1,922 & 2,270 & 2,299 & 2,537 & 2,565 & 2,851 & 2,881 & 3,189 & 3,237 & 3,637 \\
\hline Revenue & 7.9 & 8.2 & 7.7 & 9.0 & 9.2 & 10.1 & 10.3 & 11.0 & 11.0 & 11.5 \\
\hline Wages & 2.0 & 2.3 & 2.5 & 2.7 & 3.5 & 2.6 & 3.8 & 2.3 & 3.4 & 3.6 \\
\hline Current primary expenditure (cash basis) & 5.3 & 6.9 & 7.2 & 6.6 & 7.9 & 6.4 & 7.7 & 5.9 & 7.1 & 7.2 \\
\hline Investment spending $10 /$ & 1.0 & 2.6 & 2.7 & 6.9 & 8.1 & 9.6 & 13.7 & 11.5 & 10.8 & 10.3 \\
\hline Overall balance (commitment basis) & -2.0 & -3.1 & -3.9 & -4.3 & -4.2 & -3.3 & -5.0 & -1.7 & -2.4 & -1.8 \\
\hline Domestic primary cash balance $5 /$ & 1.4 & 0.5 & -0.2 & 2.1 & 1.0 & 3.3 & 2.3 & 4.7 & 3.6 & 4.0 \\
\hline Overall consolidated cash balance 11/ & -0.1 & -1.4 & -2.0 & -3.0 & -3.2 & -1.5 & -3.2 & 0.3 & -0.5 & -0.2 \\
\hline
\end{tabular}

Sources: Congolese authorities; and IMF staff estimates and projections.

1/ Reflects revised calculation of HIPC assistance from 2002-based DSA. HIPC assistance is equal to (a) for official bilateral and commercial creditors, the difference between debt service due after a hypothetical Naples stock operation at end-2002 and debt service due after HIPC relief; and (b) for multilateral creditors, the difference between debt service due after arrears clearance operations and debt service due after HIPC relief.

2/ From 2002 onward, scheduled interest before any treatment, plus interest on the September 2002 Paris Club rescheduling. From 2003 onward, interest on the rescheduling under the enhanced HIPC Initiative.

3 / Transfers and subsidies include costs of liquidation of commercial banks.

In 2002 and 2003, includes a preliminary estimate for accumulation of arrears on utilities (CGF 12 billion per year).

The domestic primary balance is defined as revenue (excluding grants), less expenditure (excluding interest on debt, foreign-financed expenditure and HIPC-related expenditure).

6/ Internal and external arrears. In 2004, including repayment of domestic debt, to be financed by the World Bank.

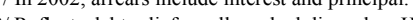

8/ Reflects debt relief on all reschedulings, less HIPC assistance, which enters the fiscal accounts as a grant.

9/ Discrepancy between monetary and fiscal dat

11/ Cash balance after interest rescheduling (including HIPC). 


\begin{tabular}{|c|c|c|c|c|c|c|c|c|c|c|}
\hline & \multirow[t]{2}{*}{2002} & \multicolumn{2}{|c|}{2003} & \multicolumn{2}{|c|}{2004} & \multicolumn{2}{|l|}{2005} & \multicolumn{2}{|c|}{2006} & \multirow{2}{*}{$\begin{array}{l}2007 \\
\text { Proj. }\end{array}$} \\
\hline & & $\begin{array}{c}\text { Country } \\
\text { Report } \\
\text { No. } 04 / 97\end{array}$ & Est. & $\begin{array}{r}\text { Country } \\
\text { Report } \\
\text { No. } 04 / 97\end{array}$ & Rev. Prog. & $\begin{array}{c}\text { Country } \\
\text { Report } \\
\text { No. } 04 / 97\end{array}$ & Proj. & $\begin{array}{c}\text { Country } \\
\text { Report } \\
\text { No. } 04 / 97\end{array}$ & Proj. & \\
\hline Total revenue and grants & 8.3 & 10.1 & 9.7 & 13.2 & 15.0 & 16.9 & 20.2 & 19.8 & 19.3 & 19.5 \\
\hline Total revenue & 7.9 & 8.2 & 7.7 & 9.0 & 9.2 & 10.1 & 10.3 & 11.0 & 11.0 & 11.5 \\
\hline Customs and excise (OFIDA) & 2.8 & 3.4 & 3.2 & 4.3 & 4.2 & 4.8 & 4.8 & 5.2 & 5.1 & 5.7 \\
\hline Direct and indirect taxes (DGC) & 2.1 & 2.2 & 2.1 & 2.5 & 2.5 & 3.0 & 3.0 & 3.7 & 3.6 & 3.7 \\
\hline Petroleum (royalties and taxes) & 1.6 & 1.6 & 1.6 & 1.2 & 1.4 & 1.1 & 1.3 & 0.9 & 1.1 & 0.9 \\
\hline Off-budget revenue & 0.6 & 0.1 & 0.1 & 0.0 & 0.0 & 0.0 & 0.0 & 0.0 & 0.0 & 0.0 \\
\hline Other & 0.7 & 0.8 & 0.7 & 1.0 & 1.0 & 1.1 & 1.2 & 1.2 & 1.3 & 1.3 \\
\hline Total grants & 0.4 & 1.9 & 2.0 & 4.3 & 5.9 & 6.8 & 10.0 & 8.8 & 8.2 & 8.0 \\
\hline Of which: HIPC assistance 1/ & $\ldots$ & 0.6 & 0.3 & 1.7 & 1.1 & 2.8 & 2.7 & 4.0 & 4.0 & 4.2 \\
\hline Total expenditure & 10.3 & 13.2 & 13.6 & 17.6 & 19.3 & 20.2 & 25.3 & 21.5 & 21.6 & 21.4 \\
\hline Current expenditure & 8.8 & 10.5 & 10.8 & 9.8 & 10.3 & 9.7 & 10.6 & 9.0 & 9.9 & 9.6 \\
\hline Wages & 2.0 & 2.3 & 2.5 & 2.7 & 3.5 & 2.6 & 3.8 & 2.3 & 3.4 & 3.6 \\
\hline Interest due 2/ & 3.2 & 3.4 & 3.4 & 3.3 & 3.1 & 3.4 & 3.1 & 3.2 & 2.9 & 2.6 \\
\hline Transfers and subsidies $3 /$ & 0.3 & 0.9 & 0.9 & 0.5 & 0.6 & 0.5 & 0.6 & 0.6 & 0.7 & 0.7 \\
\hline Other current expenditure & 3.2 & 3.9 & 4.0 & 3.4 & 3.2 & 3.2 & 3.1 & 2.9 & 2.9 & 2.8 \\
\hline Of which: centralized payments $4 /$ & 0.8 & 1.1 & 1.0 & 1.0 & 0.9 & 1.0 & 0.8 & 0.9 & 0.8 & 0.7 \\
\hline Off-budget expenditure & 0.6 & 0.1 & 0.1 & 0.0 & 0.0 & 0.0 & 0.0 & 0.0 & 0.0 & 0.0 \\
\hline Capital expenditure & 0.5 & 2.6 & 2.7 & 6.0 & 7.3 & 8.6 & 12.8 & 10.5 & 9.8 & 8.9 \\
\hline Foreign-financed & 0.3 & 1.9 & 2.1 & 5.7 & 7.0 & 8.3 & 12.4 & 10.1 & 9.5 & 8.5 \\
\hline Domestic-financed & 0.2 & 0.7 & 0.6 & 0.3 & 0.3 & 0.3 & 0.3 & 0.3 & 0.3 & 0.3 \\
\hline Other operations & 0.0 & 0.0 & 0.0 & 0.0 & 0.0 & 0.0 & 0.0 & 0.0 & 0.0 & 0.0 \\
\hline Guarantee and Contingency Fund & 0.0 & 0.0 & 0.0 & 0.0 & 0.0 & 0.0 & 0.0 & 0.0 & 0.0 & 0.0 \\
\hline Net lending & 0.4 & 0.0 & 0.0 & 0.0 & 0.0 & 0.0 & 0.0 & 0.0 & 0.0 & 0.0 \\
\hline HIPC-related expenditure & $\ldots$ & 0.0 & 0.0 & 1.7 & 1.7 & 1.9 & 1.9 & 2.0 & 1.9 & 2.9 \\
\hline Overall balance (commitment basis) & -2.0 & -3.1 & -3.9 & -4.3 & -4.2 & -3.3 & -5.0 & -1.7 & -2.4 & -1.8 \\
\hline Domestic primary balance (commitment basis) $5 /$ & 1.1 & 0.4 & -0.4 & 2.1 & 1.6 & 3.4 & 2.4 & 4.8 & 3.7 & 4.2 \\
\hline Change in arrears $6 /$ & 0.2 & 0.2 & 0.2 & -0.1 & -0.6 & -0.1 & -0.1 & -0.1 & -0.1 & -0.2 \\
\hline Overall balance (cash basis, before interest rescheduling) & -1.8 & -2.9 & -3.7 & -4.4 & -4.9 & -3.5 & -5.2 & -1.8 & -2.5 & -2.0 \\
\hline Domestic primary balance (cash basis) 5/ & 1.4 & 0.5 & -0.2 & 2.1 & 1.0 & 3.3 & 2.3 & 4.7 & 3.6 & 4.0 \\
\hline Central bank operational result & -0.7 & -0.7 & -0.8 & -0.6 & -0.6 & -0.5 & -0.5 & -0.4 & -0.4 & -0.4 \\
\hline $\begin{array}{l}\text { Overall consolidated balance (cash basis, } \\
\text { before interest rescheduling) }\end{array}$ & -2.4 & -3.6 & -4.5 & -5.1 & -5.5 & -4.0 & -5.7 & -2.2 & -2.9 & -2.4 \\
\hline
\end{tabular}




\begin{tabular}{|c|c|c|c|c|c|c|c|c|c|c|}
\hline & \multirow[t]{2}{*}{2002} & \multicolumn{2}{|c|}{2003} & \multicolumn{2}{|c|}{2004} & \multicolumn{2}{|l|}{2005} & \multicolumn{2}{|c|}{2006} & \multirow{2}{*}{$\begin{array}{l}2007 \\
\text { Proj. }\end{array}$} \\
\hline & & $\begin{array}{c}\text { Country } \\
\text { Report } \\
\text { No. } 04 / 97\end{array}$ & Est. & $\begin{array}{r}\text { Country } \\
\text { Report } \\
\text { No. } 04 / 97\end{array}$ & Rev. prog. & $\begin{array}{c}\text { Country } \\
\text { Report } \\
\text { No. 04/97 }\end{array}$ & Proj. & $\begin{array}{c}\text { Country } \\
\text { Report } \\
\text { No. } 04 / 97\end{array}$ & Proj. & \\
\hline Total financing & 2.7 & 3.4 & 4.5 & 5.1 & 5.5 & 4.0 & 5.7 & 2.2 & 2.9 & 2.4 \\
\hline Domestic financing & -0.9 & 0.4 & 1.2 & 0.0 & -0.6 & 0.0 & 0.0 & 0.0 & 0.0 & 0.0 \\
\hline Banks & -0.5 & 0.4 & 1.2 & 0.0 & -0.6 & 0.0 & 0.0 & 0.0 & 0.0 & 0.0 \\
\hline Nonbanks (certificates of deposit) & -0.4 & 0.0 & 0.0 & 0.0 & 0.0 & 0.0 & 0.0 & 0.0 & 0.0 & 0.0 \\
\hline Foreign financing & 3.6 & 3.0 & 3.3 & 5.1 & 6.1 & 4.0 & 5.7 & 2.2 & 2.9 & 2.4 \\
\hline Non domestic non resident banks & 0.4 & 0.0 & 0.0 & 0.0 & 0.0 & 0.0 & 0.0 & 0.0 & 0.0 & 0.0 \\
\hline Amortization due before debt relief & -4.7 & -4.2 & -4.1 & -3.3 & -3.0 & -4.5 & -4.0 & -6.2 & -5.6 & -5.3 \\
\hline Variation of arrears $7 /$ & -187.3 & 0.0 & 0.0 & 0.0 & 0.0 & 0.0 & 0.0 & 0.0 & 0.0 & 0.0 \\
\hline New loans & 1.3 & 2.7 & 2.4 & 5.8 & 5.6 & 6.5 & 7.2 & 7.4 & 6.5 & 5.8 \\
\hline Financing gap before debt relief & -193.7 & -4.7 & -5.0 & -2.5 & -3.6 & -2.0 & -2.5 & -1.0 & -2.0 & -1.8 \\
\hline Arrears consolidation $7 /$ & 105.7 & 0.0 & 0.0 & 0.0 & 0.0 & 0.0 & 0.0 & 0.0 & 0.0 & 0.0 \\
\hline Arrears cancellation $7 /$ & 81.5 & 0.0 & 0.0 & 0.0 & 0.0 & 0.0 & 0.0 & 0.0 & 0.0 & 0.0 \\
\hline Debt relief before HIPC assistance 8/ & 6.7 & 4.6 & 5.0 & 2.5 & 3.6 & 2.0 & 2.5 & 1.0 & 2.0 & 1.8 \\
\hline Financing gap after debt relief & 0.0 & 0.0 & 0.0 & 0.0 & 0.0 & 0.0 & 0.0 & 0.0 & 0.0 & 0.0 \\
\hline Discrepancy 9/ & 0.3 & -0.1 & 0.0 & 0.0 & 0.0 & 0.0 & 0.0 & 0.0 & 0.0 & 0.0 \\
\hline \multicolumn{11}{|l|}{ Memorandum items: } \\
\hline GDP (in billions of Congo francs) & $1,922.2$ & $2,270.1$ & $2,298.7$ & $2,536.8$ & $2,564.7$ & $2,850.8$ & $2,881.5$ & $3,188.8$ & $3,237.4$ & $3,637.2$ \\
\hline Current primary expenditure (cash basis) & 5.3 & 6.9 & 7.2 & 6.6 & 7.9 & 6.4 & 7.7 & 5.9 & 7.1 & 7.2 \\
\hline Domestic primary cash balance & 1.4 & 0.5 & -0.2 & 2.1 & 1.0 & 3.3 & 2.3 & 4.7 & 3.6 & 4.0 \\
\hline Overall consolidated cash balance $10 /$ & -0.1 & -1.4 & -2.0 & -3.0 & -3.2 & -1.5 & -3.2 & 0.3 & -0.5 & -0.2 \\
\hline
\end{tabular}

Sources: Congolese authorities; and IMF staff estimates and projections.

1/ Reflects revised calculation of HIPC assistance from 2002-based DSA. HIPC assistance is equal to (a) for official bilateral and commercial creditors, the difference between debt service due after a hypothetical Naples stock operation at end-2002 and debt service due after HIPC relief; and (b) for multilateral creditors, the difference between debt service due after arrears clearance operations and debt service due after HIPC relief.

2/ From 2002 onward, scheduled interest before any treatment, plus interest on the September 2002 Paris Club rescheduling. From 2003 onward, interest on the rescheduling under the enhanced HIPC Initiative.

3/ Transfers and subsidies include costs of liquidation of commercial banks.

4/ In 2002 and 2003, includes a preliminary estimate for accumulation of arrears on utilities (CGF 12 billion).

5/ The domestic primary balance is defined as revenue (excluding grants), less expenditure (excluding interest on debt, foreign-financed expenditure and HIPC-related expenditure).

$6 /$ Internal and external arrears. External arrears accruing in the first months of 2002 before the debt relief operations are not shown as they are consolidated during the same year. In 2004 , including repayment of domestic debt, financed by the World Bank.

7/ In 2002, arrears include interest and principal.

8/ Reflects debt relief on all reschedulings, less HIPC assistance, which enters the fiscal accounts as a grant.

9/ Discrepancy between monetary and fiscal data.

10/ Cash balance after interest rescheduling (including HIPC). 
Table 5. Democratic Republic of the Congo: Monetary Survey, 2002-04

\begin{tabular}{|c|c|c|c|c|c|}
\hline & \multirow[t]{2}{*}{2002} & \multicolumn{2}{|c|}{2003} & \multicolumn{2}{|c|}{2004} \\
\hline & & $\begin{array}{c}\text { Country } \\
\text { Report } \\
\text { No. } 04 / 97\end{array}$ & Act. & $\begin{array}{c}\text { Country } \\
\text { Report } \\
\text { No. } 04 / 97\end{array}$ & Rev. Prog \\
\hline & \multicolumn{5}{|c|}{ (In millions of Congo francs) } \\
\hline Net foreign assets & $-174,111$ & $-164,519$ & $-186,486$ & $-138,298$ & $-145,636$ \\
\hline Central bank & $-190,944$ & $-193,684$ & $-216,702$ & $-178,438$ & $-187,470$ \\
\hline Commercial banks & 16,833 & 29,165 & 30,216 & 40,141 & 41,834 \\
\hline Net domestic credit & 14,184 & 30,317 & 48,399 & 37,086 & 47,112 \\
\hline Net credit to government & 2,328 & 11,872 & 30,423 & 11,872 & 14,364 \\
\hline Credit to the private sector & 11,177 & 18,145 & 17,785 & 24,714 & 30,973 \\
\hline Credit to the parastatals & 679 & 300 & 192 & 500 & 1,775 \\
\hline Broad money (M2) & 87,626 & 114,949 & 116,267 & 143,686 & 149,636 \\
\hline Narrow money (M1) & 57,893 & 69,120 & 72,110 & 91,706 & 92,206 \\
\hline Currency in circulation & 49,757 & 60,506 & 63,148 & 80506 & 80,506 \\
\hline Demand deposits & 8,135 & 8614 & 8,962 & 11200 & 11700 \\
\hline Quasi money & 29,733 & 45,829 & 44,157 & 51,980 & 57,430 \\
\hline Time deposits in domestic currency & 162 & 845 & 321 & 900 & 900 \\
\hline Foreign currency deposits & 29,571 & 44,984 & 43,835 & 51,080 & 56,530 \\
\hline Import deposits & 3,893 & 6,076 & 4,728 & 10,330 & 8,500 \\
\hline Other items, net (including valuat. change) & $-251,447$ & $-255,227$ & $-259,081$ & $-255,227$ & $-256,660$ \\
\hline \multirow[t]{2}{*}{ Of which: Valuation change } & 60,855 & 61,521 & 84,327 & 61,521 & 84,327 \\
\hline & \multicolumn{5}{|c|}{ (Annual change in percent) } \\
\hline Net foreign assets & 4.0 & 5.5 & -7.1 & 15.9 & 21.9 \\
\hline Net domestic credit & -39.5 & 113.7 & 241.2 & 22.3 & -2.7 \\
\hline Net credit to government & -81.0 & 410.0 & $1,206.8$ & 0.0 & -52.8 \\
\hline Credit to the private sector & 3.6 & 62.3 & 59.1 & 36.2 & 74.2 \\
\hline Credit to the parastatals & 71.7 & -55.8 & -71.7 & 66.7 & 825.0 \\
\hline Broad money (M2) & 25.7 & 31.2 & 32.7 & 25.0 & 28.7 \\
\hline Narrow money (M1) & 35.8 & 19.4 & 24.6 & 32.7 & 27.9 \\
\hline Currency in circulation & 56.1 & 21.6 & 26.9 & 33.1 & 27.5 \\
\hline Demand deposits & -24.3 & 5.9 & 10.2 & 30.0 & 30.6 \\
\hline Quasi money & 9.9 & 54.1 & 48.5 & 13.4 & 30.1 \\
\hline Time deposits in domestic currency & 547.4 & 422.9 & 98.8 & 6.5 & 180.2 \\
\hline Foreign currency deposits & 9.4 & 52.1 & 48.2 & 13.6 & 29.0 \\
\hline Import deposits & -38.7 & 56.1 & 21.4 & 70.0 & 79.8 \\
\hline \multirow[t]{2}{*}{ Other items, net } & -7.5 & -1.5 & -3.0 & 0.0 & 0.9 \\
\hline & \multicolumn{5}{|c|}{$\begin{array}{l}\text { (Annual change in percent of beginning -of-period } \\
\text { broad money; unless otherwise indicated) }\end{array}$} \\
\hline Net foreign assets & 10.4 & 10.9 & -14.1 & 22.8 & 35.1 \\
\hline Net domestic credit & -13.3 & 18.4 & 39.0 & 5.9 & -1.1 \\
\hline Net credit to government & -14.2 & 10.9 & 32.1 & 0.0 & -13.8 \\
\hline Credit to the private sector & 0.6 & 8.0 & 7.5 & 5.7 & 11.3 \\
\hline Credit to the parastatals & 0.4 & -0.4 & -0.6 & 0.2 & 1.4 \\
\hline Broad money (M2) & 25.7 & 31.2 & 32.7 & 25.0 & 28.7 \\
\hline Narrow money (M1) & 21.9 & 12.8 & 16.2 & 19.6 & 17.3 \\
\hline Currency in circulation & 25.7 & 12.3 & 15.3 & 17.4 & 14.9 \\
\hline Demand deposits & -3.8 & 0.5 & 0.9 & 2.2 & 2.4 \\
\hline Quasi money & 3.8 & 18.4 & 16.5 & 5.4 & 11.4 \\
\hline Time deposits in domestic currency & 0.2 & 0.8 & 0.2 & 0.0 & 0.5 \\
\hline Foreign currency deposits & 3.6 & 17.6 & 16.3 & 5.3 & 10.9 \\
\hline Import deposits & -3.5 & 2.5 & 1.0 & 3.7 & 3.2 \\
\hline Other items, net & -25.1 & -4.3 & -8.7 & 0.0 & 2.1 \\
\hline \multicolumn{6}{|l|}{ Memorandum items: } \\
\hline Velocity (GDP/ broad money) & 21.9 & 19.7 & 19.8 & 17.7 & 17.1 \\
\hline Net foreign assets (in millions of U.S. dollars) & -555.2 & -524.6 & -594.7 & -441.0 & -464.4 \\
\hline Of which: central bank & -608.9 & -617.6 & -691.0 & -569.0 & -597.8 \\
\hline
\end{tabular}

Sources: Congolese authorities; and IMF staff estimates and projections. 
Table 6. Democratic Republic of the Congo: Balance of Payments Summary, 2002-07

\begin{tabular}{|c|c|c|c|c|c|c|c|c|c|c|}
\hline & \multirow[t]{2}{*}{2002} & \multicolumn{2}{|l|}{2003} & \multicolumn{2}{|c|}{2004} & \multicolumn{2}{|l|}{2005} & \multicolumn{2}{|l|}{2006} & \multirow{2}{*}{$\begin{array}{l}2007 \\
\text { Proj. }\end{array}$} \\
\hline & & $\begin{array}{c}\text { Country } \\
\text { Report } \\
\text { No. } 04 / 97\end{array}$ & Est. & $\begin{array}{c}\text { Country } \\
\text { Report } \\
\text { No. } 04 / 97\end{array}$ & Rev. Prog. & $\begin{array}{c}\text { Country } \\
\text { Report } \\
\text { No. } 04 / 97\end{array}$ & Proj. & $\begin{array}{c}\text { Country } \\
\text { Report } \\
\text { No. } 04 / 97\end{array}$ & Proj. & \\
\hline & \multicolumn{10}{|c|}{ (In millions of U.S. dollars, unless otherwise indicated) } \\
\hline Current account & -152 & -112 & 35 & -367 & -225 & -507 & -474 & -550 & -435 & -464 \\
\hline Merchandise trade & -17 & -128 & -123 & -362 & -460 & -466 & -780 & -517 & -602 & -666 \\
\hline Exports, f.o.b. & 1,076 & 1,242 & 1,281 & 1,375 & 1,413 & 1,534 & 1,485 & 1,706 & 1,614 & 1,768 \\
\hline Of which: diamonds & 653 & 798 & 813 & 859 & 857 & 958 & 932 & 1,090 & 1,014 & 1,117 \\
\hline Imports, f.o.b. & $-1,093$ & $-1,370$ & $-1,404$ & $-1,738$ & $-1,873$ & $-2,000$ & $-2,265$ & $-2,223$ & $-2,216$ & $-2,434$ \\
\hline Of which: aid-related imports & -270 & -354 & -353 & -529 & -659 & -637 & -878 & -748 & -734 & -685 \\
\hline Services & -255 & -260 & -249 & -339 & -361 & -410 & -485 & -461 & -422 & -414 \\
\hline Receipts & 99 & 142 & 148 & 154 & 176 & 164 & 196 & 175 & 201 & 211 \\
\hline Expenditure & -354 & -402 & -397 & -494 & -537 & -574 & -681 & -636 & -624 & -626 \\
\hline Of which: aid-related imports & -109 & -125 & -126 & -178 & -213 & -218 & -311 & -245 & -241 & -191 \\
\hline Income & -297 & -217 & -173 & -274 & -226 & -339 & -286 & -347 & -296 & -240 \\
\hline Receipts & 20 & 23 & 68 & 24 & 72 & 24 & 75 & 25 & 77 & 78 \\
\hline Expenditure & -318 & -240 & -242 & -297 & -298 & -363 & -361 & -371 & -373 & -318 \\
\hline Of which: interest payments $1 /$ & -285 & -195 & -196 & -204 & -207 & -228 & -230 & -233 & -233 & -226 \\
\hline Current transfers & 417 & 493 & 581 & 608 & 822 & 709 & 1,078 & 775 & 885 & 857 \\
\hline Of which: Official aid & 415 & 484 & 494 & 539 & 675 & 616 & 894 & 658 & 657 & 581 \\
\hline Capital and financial account & 150 & -151 & -97 & 187 & 33 & 291 & 149 & 261 & 98 & 314 \\
\hline Official capital & 137 & -79 & -91 & 156 & 171 & 134 & 232 & 86 & 69 & 51 \\
\hline Gross disbursements & 400 & 160 & 148 & 357 & 375 & 431 & 525 & 537 & 517 & 509 \\
\hline Of which: net new financing & 74 & 160 & 148 & 357 & 375 & 431 & 525 & 537 & 517 & 509 \\
\hline Scheduled amortization 2/ & -263 & -239 & -239 & -200 & -203 & -297 & -293 & -450 & -448 & -458 \\
\hline Private capital (net) & 13 & -73 & -6 & 31 & -139 & 157 & -83 & 175 & 29 & 262 \\
\hline Of which: foreign direct investment & 117 & 158 & 132 & 197 & 204 & 317 & 320 & 280 & 386 & 343 \\
\hline Balance before errors and omissions & -2 & -263 & -61 & -180 & -192 & -216 & -325 & -289 & -337 & -150 \\
\hline Errors and omissions & -159 & 0 & -287 & 0 & 0 & 0 & 0 & 0 & 0 & 0 \\
\hline Overall balance & -161 & -263 & -348 & -180 & -192 & -216 & -325 & -289 & -337 & -150 \\
\hline
\end{tabular}


2002

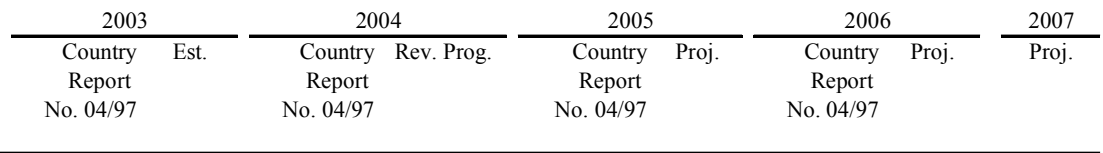

\section{Financing}

Net change in non-Fund arrears

Net banking sector reserves (increase, - )

Of which: net Fund credit

Financing gap before exceptional assistance

Exceptional financing

Consolidation of arrears

Debt relief on current debt service

Relief from Naples flow rescheduling

Relief from Cologne flow rescheduling and the capitalization of moratorium interest 3/

Relief from HIPC assistance by multilateral creditors in the form of grants

Relief from a stock operation at the completion point $4 /$

(In millions of U.S. dollars, unless otherwise indicated)

$\begin{array}{rrrrrrrrrr}-10,169 & -89 & -20 & -489 & -386 & -104 & -63 & -81 & -145 & -368 \\ -10,142 & -59 & -59 & -406 & -256 & 0 & 0 & 0 & 0 & 0 \\ -26 & -31 & 39 & -84 & -130 & -104 & -63 & -81 & -145 & -368 \\ 21 & 74 & 75 & 74 & 79 & 74 & 79 & 0 & 0 & -62 \\ & & & & & & & & & \\ -10,330 & -353 & -368 & -669 & -579 & -320 & -388 & -370 & -482 & -518 \\ & & & & & & & & & \\ 10,330 & 353 & 368 & 669 & 579 & 320 & 388 & 370 & 482 & 518 \\ 9,960 & 88 & 88 & 411 & 281 & 0 & 0 & 0 & 0 & 0 \\ 370 & 264 & 279 & 258 & 298 & 320 & 388 & 370 & 482 & 518 \\ 273 & 118 & 118 & 57 & 57 & 32 & 32 & 0 & 0 & 0 \\ 97 & 130 & 145 & 156 & 196 & 236 & 304 & 218 & 286 & 0 \\ 0 & 17 & 17 & 45 & 45 & 53 & 53 & 66 & 66 & 84 \\ 0 & 0 & 0 & 0 & 0 & 0 & 0 & 85 & 129 & 434\end{array}$

(In percent of GDP; unless otherwise indicated)

\section{Memorandum items:}

Debt service, after debt relief (percentage of exports of goods and services) 5/

Current account balance, including grants, before debt relief

Current account balance, excluding official transfers, before debt relief

Current account balance, including grants, after debt relief

Current account balance, excluding grants, after debt relief

Gross official reserves (millions of U.S. dollars)

In weeks of non-aid-related imports of goods and nonfactor services

In weeks of official non-aid-related imports of goods and nonfactor services

HIPC Initiative assistance 6

\begin{tabular}{rrrrrrrrrr}
2.9 & 10.2 & 8.9 & 9.4 & 5.8 & 12.3 & 8.0 & 16.9 & 10.9 & 11.5 \\
-2.8 & -2.0 & 0.6 & -6.0 & -3.3 & -7.6 & -6.5 & -7.6 & -5.4 & -5.3 \\
-10.3 & -10.7 & -8.1 & -14.7 & -13.3 & -16.8 & -18.7 & -16.7 & -13.7 & -12.0 \\
2.2 & -1.1 & 1.2 & -4.1 & -2.0 & -4.7 & -3.6 & -3.5 & -1.5 & -1.2 \\
-5.3 & -9.7 & -7.5 & -12.8 & -12.0 & -14.0 & -15.8 & -12.6 & -9.7 & -7.8 \\
74.7 & 156.6 & 96.6 & 272.1 & 209.3 & 382.2 & 318.2 & 479.1 & 479.1 & 611.9 \\
2.9 & 5.3 & 3.3 & 8.2 & 6.2 & 10.7 & 8.9 & 12.1 & 11.4 & 14.1 \\
3.3 & 5.9 & 3.5 & 9.1 & 6.6 & 11.8 & 9.3 & 13.1 & 11.8 & 14.5 \\
0.0 & 35.0 & 17.0 & 107.4 & 75.5 & 185.3 & 201.4 & 293.0 & 317.4 & 362.4 \\
\hline
\end{tabular}

Sources: Congolese authorities; and IMF staff estimates and projections.

1/ Including interest due to the IMF.

2/ Excluding repayments to the IMF.

3/ The amounts for the revised program and current projections include additional debt relief beyond Cologne terms.

4/ The current projection includes the impact on debt service of additional cancellation of debt committed by individual creditors beyond the HIPC Initiative.

5/ Takes into account the Naples flow rescheduling, the capitalization of moratorium interest, the Cologne flow rescheduling, the additional debt service relief beyond the Cologne flow rescheduling, the grants provided by multilateral creditors in the context of the enhanced HIPC Initiative and the impact of the stock-of-debt operation (including any additional cancellation beyond the HIPC Initiative) at the completion point on debt service. The data also take into account the rescheduling agreements that have already been signed with some commercial creditors as well as the impact on debt service of the accumulation of arrears to non-Paris Club creditors.

6/ For bilateral and commercial creditors, including the debt relief resulting from the enhanced HIPC Initiative (excluding debt relief beyond the HIPC Initiative) following the use of all traditional debt relief mechanisms, including a hypothetical stock-of-debt operation on Naples terms. Only assistance based on rescheduling agreements which have been concluded are taken into account. For multilateral creditors, including the amount of

HIPC grants that are made available for the purpose of delivering their share of assistance under the Initiative.

\section{CInternational Monetary Fund. Not for Redistribution}


Table 7. Democratic Republic of the Congo: Nominal Stock of External Public Debt and Net Present Value of External Public Debt, by Creditor Group, End-2003 1/

\begin{tabular}{|c|c|c|c|c|}
\hline & \multicolumn{2}{|c|}{ Nominal Debt Stock 2/ } & \multicolumn{2}{|c|}{$\begin{array}{c}\text { Net Present Value } \\
\text { of Debt 3/ }\end{array}$} \\
\hline & $\begin{array}{l}\text { Millions of } \\
\text { U.S. dollars }\end{array}$ & $\begin{array}{r}\text { Percent } \\
\text { of total }\end{array}$ & $\begin{array}{l}\text { Millions of } \\
\text { U.S. dollars }\end{array}$ & $\begin{array}{l}\text { Percent } \\
\text { of total }\end{array}$ \\
\hline Total & $10,550.3$ & 100.0 & $7,883.3$ & 100.0 \\
\hline Multilateral & $3,732.0$ & 35.4 & $2,639.2$ & 33.5 \\
\hline World Bank & $1,634.3$ & 15.5 & 865.8 & 11.0 \\
\hline African Development Bank Group & $1,130.7$ & 10.7 & 739.9 & 9.4 \\
\hline IMF & 703.4 & 6.7 & 505.0 & 6.4 \\
\hline Other multilateral & 263.6 & 2.5 & 528.5 & 6.7 \\
\hline Bilateral and commercial & $6,818.3$ & 64.6 & $5,244.1$ & 66.5 \\
\hline Paris Club & $6,116.8$ & 58.0 & $4,571.4$ & 58.0 \\
\hline Other official bilateral & 441.0 & 4.2 & 421.2 & 5.3 \\
\hline Commercial & 260.5 & 2.5 & 251.4 & 3.2 \\
\hline
\end{tabular}

Sources: Congolese authorities; IMF staff estimates; and end-2002 debt sustainability analysis.

1/ Preliminary estimates.

2/ Includes the stock of debt outstanding at the base year (i.e., in 2002) after Naples rescheduling, outstanding arrears, rescheduling, and new debt disbursed since 2002.

3/ Before rescheduling and HIPC debt relief. Estimates do not take into account changes in the discount rate. 
Table 8. Democratic Republic of the Congo: Debt Service on External Debt, 2002-07

(In millions of U.S. dollars; unless otherwise indicated)

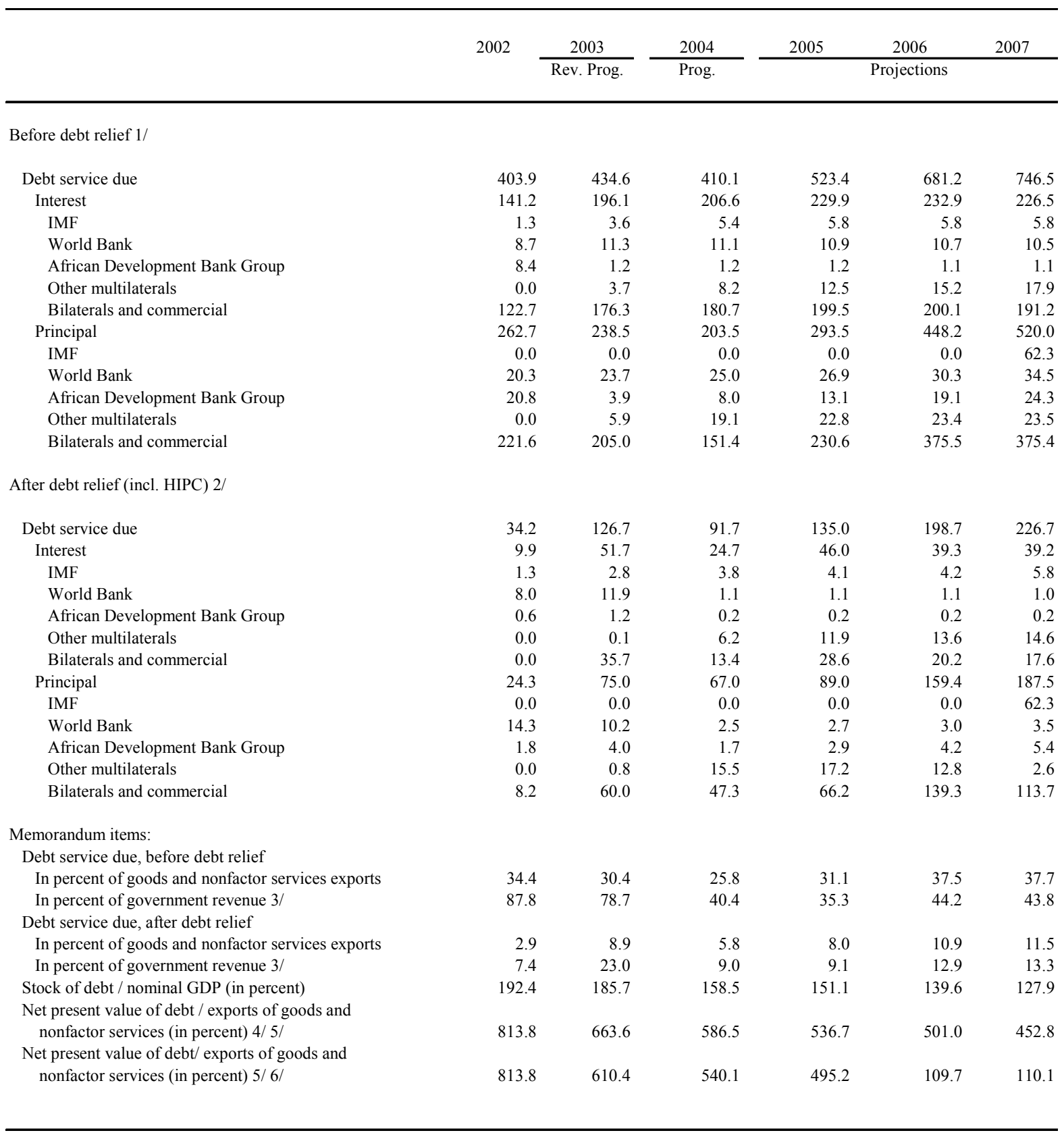

Sources: Congolese authorities; IMF staff estimates and projections; and end-2002 debt sustainability analysis (DSA).

1/ Reflects debt service on current maturities, including rescheduling and new financing. Based on end-2002 DSA data.

2/ Reflects debt relief in the form of flow rescheduling, as well as HIPC grants by multilateral creditors. Also including the accumulation of arrears to non-Paris Club and commercial creditors, pending the conclusion of rescheduling agreements.

$3 /$ Including grants.

4/ Before rescheduling and HIPC debt relief. The amounts at end-2002 reflect the Naples rescheduling agreement.

5/ Three-year backward average of exports of goods and nonfactor services.

6/ After rescheduling and HIPC debt relief. The amounts also include the accumulation of arrears to non-Paris Club creditors. The amounts at end-

2002 reflect the Naples rescheduling agreement.

\section{CInternational Monetary Fund. Not for Redistribution}


Table 9. Democratic Republic of the Congo: External Financing Requirements and Sources, 2002-07 (In millions of U.S. dollars)

\begin{tabular}{|c|c|c|c|c|c|c|}
\hline & 2002 & 2003 & 2004 & 2005 & 2006 & 2007 \\
\hline & & Rev. prog. & Prog. & \multicolumn{3}{|c|}{ Projections } \\
\hline Noninterest current account $2 /$ & -282 & -263 & -694 & $-1,138$ & -859 & -818 \\
\hline Resource balance & -272 & -372 & -821 & $-1,265$ & $-1,024$ & $-1,081$ \\
\hline Exports of goods and nonfactor services & 1,174 & 1,429 & 1,589 & 1,681 & 1,816 & 1,979 \\
\hline Imports of goods and nonfactor services & $-1,447$ & $-1,801$ & $-2,410$ & $-2,946$ & $-2,840$ & $-3,060$ \\
\hline Income 1/ & -12 & 23 & -19 & -56 & -63 & -14 \\
\hline Receipts & 20 & 68 & 72 & 75 & 77 & 78 \\
\hline Expenditure & -32 & -45 & -91 & -131 & -140 & -92 \\
\hline Current transfers, net $2 /$ & 2 & 87 & 146 & 184 & 228 & 276 \\
\hline Public & 48 & 29 & 35 & 42 & 49 & 57 \\
\hline Private & -46 & 57 & 111 & 142 & 179 & 220 \\
\hline Interest on public debt & -285 & -196 & -207 & -230 & -233 & -226 \\
\hline Capital and financial account 3/ & -250 & -244 & -342 & -376 & -420 & -196 \\
\hline Official capital & -263 & -239 & -203 & -293 & -448 & -458 \\
\hline Of which: amortization & -263 & -239 & -203 & -293 & -448 & -458 \\
\hline Private capital (net) & 13 & -6 & -139 & -83 & 29 & 262 \\
\hline Change in net reserves (increase, -) 4/ & -47 & -35 & -209 & -142 & -145 & -306 \\
\hline Errors and omissions & -159 & -287 & 0 & 0 & 0 & 0 \\
\hline External financing requirements & $-1,024$ & $-1,025$ & $-1,452$ & $-1,886$ & $-1,656$ & $-1,546$ \\
\hline \multicolumn{7}{|l|}{ Sources of financing } \\
\hline Humanitarian assistance & 229 & 229 & 195 & 189 & 156 & 146 \\
\hline UN humanitarian assistance & 114 & 135 & 131 & 127 & 95 & 85 \\
\hline Bilateral humanitarian assistance & 116 & 94 & 64 & 63 & 61 & 61 \\
\hline Financing gap after humanitarian assistance & -795 & -797 & $-1,257$ & $-1,697$ & $-1,500$ & $-1,399$ \\
\hline Net change in arrears & $-10,142$ & -59 & -256 & 0 & 0 & 0 \\
\hline Financing gap after net change in arrears & $-10,937$ & -856 & $-1,513$ & $-1,697$ & $-1,500$ & $-1,399$ \\
\hline Consolidation of arrears & 9,960 & 88 & 281 & 0 & 0 & 0 \\
\hline Financing gap after consolidation of arrears & -977 & -767 & $-1,232$ & $-1,697$ & $-1,500$ & $-1,399$ \\
\hline Assistance on debt service & 370 & 279 & 298 & 388 & 482 & 518 \\
\hline Naples flow rescheduling & 273 & 118 & 57 & 32 & 0 & 0 \\
\hline Cologne flow rescheduling and capitalization of moratorium interest 6/ & 96 & 145 & 196 & 304 & 286 & 0 \\
\hline HIPC grants & 0 & 17 & 45 & 53 & 66 & 84 \\
\hline Debt relief from a stock-of-debt operation & 0 & 0 & 0 & 0 & 129 & 434 \\
\hline Financing gap after assistance on debt service & -607 & -488 & -934 & $-1,308$ & $-1,018$ & -881 \\
\hline
\end{tabular}

\section{CInternational Monetary Fund. Not for Redistribution}


Table 9. Democratic Republic of the Congo: External Financing Requirements and Sources, 2002-07 (In millions of U.S. dollars)

\begin{tabular}{|c|c|c|c|c|c|c|}
\hline & \multirow{2}{*}{2002} & \multirow{2}{*}{$\frac{2003}{\text { Rev. prog. }}$} & \multirow{2}{*}{$\frac{2004}{\text { Prog. }}$} & 2005 & 2006 & 2007 \\
\hline & & & & \multicolumn{3}{|c|}{ Projections } \\
\hline Project financing 5/ & 514 & 293 & 629 & 1,087 & 918 & 843 \\
\hline Project grants & 186 & 266 & 480 & 704 & 501 & 434 \\
\hline Multilateral & 136 & 183 & 276 & 319 & 271 & 178 \\
\hline World Bank & 21 & 46 & 84 & 118 & 148 & 110 \\
\hline African Development Bank & 0 & 2 & 8 & 13 & 10 & 5 \\
\hline European Union & 66 & 77 & 128 & 133 & 72 & 27 \\
\hline Other & 49 & 58 & 56 & 54 & 41 & 36 \\
\hline Bilateral & 51 & 83 & 205 & 386 & 230 & 256 \\
\hline Project loans & 328 & 27 & 149 & 383 & 417 & 409 \\
\hline Multilateral & 326 & 26 & 143 & 360 & 309 & 294 \\
\hline World Bank & 326 & 26 & 127 & 317 & 239 & 224 \\
\hline African Development Bank & 0 & 0 & 15 & 32 & 30 & 13 \\
\hline European Union & 0 & 0 & 0 & 6 & 31 & 43 \\
\hline Other & 0 & 0 & 1 & 5 & 10 & 14 \\
\hline Bilateral & 2 & 1 & 6 & 23 & 108 & 115 \\
\hline Financing gap after project financing & -93 & -195 & -305 & -221 & -100 & -38 \\
\hline Budget and balance of payments support 5/ & 93 & 195 & 305 & 221 & 100 & 38 \\
\hline Budget grants & 0 & 0 & 0 & 0 & 0 & 0 \\
\hline Multilateral & 0 & 0 & 0 & 0 & 0 & 0 \\
\hline World Bank & 0 & 0 & 0 & 0 & 0 & 0 \\
\hline African Development Bank & 0 & 0 & 0 & 0 & 0 & 0 \\
\hline European Union & 0 & 0 & 0 & 0 & 0 & 0 \\
\hline Other & 0 & 0 & 0 & 0 & 0 & 0 \\
\hline Bilateral & 0 & 0 & 0 & 0 & 0 & 0 \\
\hline Budget loans & 93 & 195 & 305 & 221 & 100 & 38 \\
\hline Multilateral & 93 & 195 & 305 & 221 & 100 & 38 \\
\hline IMF 6/ & 21 & 75 & 79 & 79 & 0 & -62 \\
\hline World Bank & 72 & 120 & 160 & 142 & 100 & 100 \\
\hline African Development Bank & 0 & 0 & 66 & 0 & 0 & 0 \\
\hline European Union & 0 & 0 & 0 & 0 & 0 & 0 \\
\hline Other & 0 & 0 & 0 & 0 & 0 & 0 \\
\hline Bilateral & 0 & 0 & 0 & 0 & 0 & 0 \\
\hline Residual financing gap & 0 & 0 & 0 & 0 & 0 & 0 \\
\hline
\end{tabular}

Sources: Congolese authorities; IMF staff estimates and projections; and end-2002 debt sustainability analysis.

1/ Excluding interest on public debt.

2/ Excluding official aid.

3/ Excluding loan disbursements.

4/ Excluding net use of IMF credit.

5/ By implementing agency. Includes currently unidentified financing.

6/ On a net basis. 
Table 10. Democratic Republic of the Congo and Sub-Saharan Africa:

Selected Poverty and Living Standards Indicators, 2001

(In percent; unless otherwise indicated)

DRC Sub-Saharan Africa

Population, mid-year (millions)

52

674

Population growth rate

3.0

2.6

GDP per capita in U.S. dollars

99

470

Infant mortality (per thousand)

129

105

Under-five mortality rate (per 1,000 live births)

205

171

Maternal mortality ratio (per 100,000 live births)

950

Prevalence of HIV/AIDS

Males

Females

Life expectancy

Adult literacy rate

Male

Female

Gross school enrollment rates

Male

Female

Impact of conflict

Child soldiers (thousands)

Displaced persons (millions)

Deaths from deprivation (millions)

Sources: Congolese authorities, World Bank; and World Development Indicators. 
Mr. Rodrigo de Rato

Managing Director

International Monetary Fund

Washington, D.C. 20431

Dear Mr. de Rato:

1. On June 12, 2002, the IMF's Executive Board approved a three-year arrangement for the Democratic Republic of the Congo (DRC) under the Poverty Reduction and Growth Facility (PRGF). This arrangement was designed to support the Government Economic Program (PEG) for the period April 1, 2002-July 31, 2005. In accordance with this arrangement, the government of the DRC has conducted discussions with an IMF mission on the fourth program review for the period October 1, 2003 to March 31, 2004. The discussions covered program implementation during this period, as well as the outlook for, and the economic and financial measures to be implemented during, the remainder of 2004, taking into account our country's reunification. The government of the DRC remains determined to implement the policies and measures described in the interim poverty reduction strategy paper (I-PRSP), as well as in the memorandum on economic and financial policies (MEFP), which is attached to this letter, and supplements its letters of April 13,2002, February 4, 2003, July 3, 2003, and December 10, 2003.

2. We are pleased to note the broadly satisfactory implementation of the PEG in 2003, despite some budgetary difficulties encountered during the last quarter, due inter alia to the establishment of the new institutions provided for in our Transitional Constitution and some delays in the implementation of our tax reform. The government took steps in early 2004 to address these difficulties, and the results in the first quarter of 2004 exceeded expectations.

3. The government will strengthen its efforts to maintain macroeconomic stability and reconstruct the country. The Central Bank of the Congo (BCC) will continue to implement an independent monetary policy that aims to maintain price stability in the context of the floating exchange rate system. Before end-June 2004, the government will adopt a draft supplementary budget consistent with the objectives of our economic program for 2004, to take into account the implementation of our national Disarmament, Demobilization, and Reintegration (DDR) Program for the armed forces, the preparation in 2004 of the constitutional referendum, the local, legislative, and presidential elections in 2005, the cost of the restructuring of commercial banks, the repayment schedule for the cross-arrears between the government and the private sector, and updates of the effects of reunification and of external aid. This draft supplementary budget will be submitted to the National Assembly by July 2004.

4. Finally, the government will intensify its efforts to fully establish the rule of law throughout the country and ensure compliance with all the new codes, and promote good 
governance and transparency in the conduct of public affairs at the level of the central government, the national institutions, the public enterprises, and the eleven provinces of the DRC. The government intends to fight corruption at all levels, including in the mining sector (particularly diamond mining) and the forestry sector, and reduce bureaucratic "red tape."

5. The year 2004 will be marked by preparations for truly free and transparent elections at all levels for the first time in our country's history, allowing the creation of a constitutional democracy. It is our desire that these elections be all-inclusive. For that purpose, a roadmap has been prepared that we intend to follow, with assistance of the international community, in an atmosphere of calm and respect of democratic rules. To this end, the various commissions established under the Global and Inclusive Agreement and the Transitional Constitution are already at work, including the Political, Defense and Security Commission, which is focusing particularly on preparing the legal instruments for the Transition. Thus, since

September 2003 the government has approved bills on the organization and functioning of political parties, the armed forces of the DRC, and civic institutions.

6. The government has issued various decrees for these laws, which relate specifically to (a) the establishment, organization, and functioning of the Armed Forces Commission, which has as its mission to control the Armed Forces of the DRC with a view to determining their actual size; (b) the establishment, organization, and functioning of an integrated military structure; (c) the appointment within the army of the joint chiefs of staff and other key positions at the national and provincial levels; (d) the appointment of the coordinator for the implementation of the DDR program; (e) the establishment and organization of the preparatory committee for the International Conference on Peace, Security, Development and Democracy in the Great Lakes Region; and (f) the appointment of the provincial governors and deputy governors, an important step in the effective redeployment of central government authority throughout the country. In addition, the government is expected to approve the anticorruption bill in June 2004. The government approved the bill on territorial and administrative organization in early 2004, whereas the bill on money laundering has been submitted to the National Assembly. Finally, we expect that the bills necessary for the preparation of the elections, specifically the law on nationality and the decree on voter registration, will be approved soon.

7. Despite this progress on the legal front, the government will redouble its efforts to ensure that these laws are effectively implemented, both at the level of each ministry involved and at the level of each province, to ensure that the roadmap to free and democratic elections is followed. Finally, the members of government undertake to strengthen governmental solidarity and to ensure consistent governmental action during this transition period. Thus, the government will make sure that the Code of Ethics and Good Conduct is strictly applied in the public sector, including with respect to members of government. Moreover, the government intends to ensure strict execution of the 2004 budget and the supplementary budget, fully respecting the composition of expenditures, particularly as regards pro-poor spending. Finally, with the sustained assistance of the international community, in particular the forces of the United Nations Observation Mission in the Congo 
(MONUC), and, with the cooperation of neighboring countries, the government will endeavor to restore stability and security throughout the country, particularly in the northeast and east.

8. An examination of the performance criteria at end-March 2004 indicates that 3 of the 11 quantitative performance criteria have not been met, owing largely to a delay in ratifying financing agreements with the African Development Bank (AfDB) and the World Bank. Consequently, the floor on the BCC's net external assets was missed by US $\$ 77$ million. The ceiling on the BCC's net domestic assets and net bank credit to the government, adjusted for external assistance, were exceeded by 0.8 percent of GDP and 0.2 percent of GDP, respectively. These three criteria have been audited by an international auditing firm. The structural performance criterion regarding the BCC's approval of the list of commercial banks to be liquidated or restructured was met. However, only 2 of the 4 structural benchmarks were met at end-March 2004. An international audit firm to conduct the audit of the diamond company (MIBA) will be selected in September 2004 with assistance from the European Union. The structural benchmark concerning submission of the new customs code to Parliament will be met no later than June 2004.

9. As for the four structural benchmarks at end-June 2004, the benchmark on finalizing the reorganization plan for the petroleum products distribution company (COHYDRO) is expected to be executed with some delay, as is the benchmark on the completion of the strategic audit of the Public Enterprise Council (Conseil Supérieur du Portefeuille). The benchmark on finalizing the plans to reorganize commercial banks considered viable and to liquidate nonviable commercial banks has been met. Finally, the benchmark concerning the adoption of a simplified double-entry accounting framework for the government will be met.

10. Taking account of the corrective measures described in the attached memorandum, the government requests waivers from the IMF Executive Board with respect to the nonobservance of the three quantitative performance criteria referred to above. The government also requests that the Board approve an additional tranche of interim assistance under the Heavily Indebted Poor Countries (HIPC) Initiative to enable our country to continue to benefit from the HIPC Initiative during the interim period.

11. The government will provide the IMF with all information requested by the Fund on the progress in the implementation of its economic and financial policies, and the attainment of its program targets, as described in the attached technical memorandum of understanding. As in the past, the government authorizes the publication of this letter, the MEFP attached to this letter, and the associated IMF staff report. In addition, the DRC will undertake with the IMF the fifth six-monthly review of its economic program supported by the PRGF, which should be completed January 15, 2005.

12. The government considers that the policies and measures set out in the attached memorandum are adequate to achieve its program objectives. The government is prepared to take any further measures that may be necessary toward this end. Moreover, the government 
pledges to consult the IMF, whether on its own initiative or at your request, on the adoption of any measures that may prove necessary.

13. The government would like to thank the international community, and the International Monetary Fund in particular, for the support for its stabilization and reconstruction efforts, and calls upon the international community to maintain its unwavering support for the central government and provincial governments of the DRC during this critical period in the nation's history.

Sincerely yours,

$/ \mathrm{s} /$

Joseph Kabila

President of the Democratic Republic of the Congo 


\section{DEMOCRATIC REPUBLIC OF THE CONGO}

\section{Memorandum on Economic and Financial Policies for 2004}

Kinshasa, June 24, 2004

\section{Implementation of the Government ECONOMiC Program during The PeRiod OCTOBER 2003-MARCH 2004}

1. We are pleased that program implementation during the period October 2003March 2004 was broadly satisfactory, owing to our strong efforts in the first quarter of 2004 to improve public expenditure control and increase revenue collection. Progress in the implementation of structural and sectoral reforms since the beginning of the year has been satisfactory, despite delays in some areas. In view of lower-than-expected external debt service and the good results in the first quarter of 2004 (despite the civil service strike in February), we believe that the overall objectives for end-2004 remain achievable. The supplementary budget to be submitted to Parliament in July 2004 addresses the government's three major challenges, namely, the National Disarmament, Demobilization, and Reintegration (DDR) Program for ex-combatants, the cost of preparing the elections, and the repayment of domestic debt. The Technical Committee for Reform Monitoring responsible for the Government Economic Program will be expanded to facilitate the timely implementation of measures and reforms included in the program. With the support of the international community, we will also continue to strengthen the administrative capacity of the central government and of the provincial and local governments, by extending across the whole nation the structural and sectoral reforms that are currently being implemented.

2. Our estimates strongly indicate that economic growth exceeded expectations in 2003, reaching 5.6 percent instead of 5 percent (Table 1), and in the first quarter of 2004 there was a further acceleration. Thus, the rate of growth of real per capita GDP accelerated for the second year in a row. According to the National Investment Promotion Agency (ANAPI), approximately US\$1.6 billion in new domestic and foreign private investment was approved in the first quarter of 2004. All sectors of the economy are improving, except for copper and cobalt, as a result of the ongoing restructuring of the GECAMINES mining company. The diamond, construction and public works, manufacturing, transport and communications, and agricultural sectors are contributing significantly to growth. End-period inflation was lower than programmed, at 4.4 percent at end-December 2003 and 2.6 percent at end-May 2004, and continued the steep decline that began in 2001. Since the start of 2004, retail oil prices have increased by roughly 4 percent in the wake of rising prices on the international market. Moreover, the exchange rate of the Congo franc has remained stable vis-à-vis the U.S. dollar. 


\section{Government finances}

3. The overall fiscal objectives for 2003 have not been fully met, owing in large measure to a revenue shortfall ( 0.5 percent of GDP), mainly caused by the delay in submitting legislation to Parliament for the large-scale tax reform that we are implementing. Moreover, current primary expenditure has been higher than expected ( 0.3 percent of GDP), largely because of the cost of setting up new institutions as required by the Transitional Constitution and bonuses paid to judicial staff. However, pro-poor expenditure, according to the new definition that includes infrastructure, was higher than envisaged, at 1.5 percent of GDP instead of 1.2 percent. External debt service was less than projected while externally financed investment expenditure was slightly higher. Consequently, the domestic primary balance on a cash basis showed a deficit of 0.2 percent of GDP against a programmed surplus of 0.5 percent, whereas the consolidated overall balance (on a cash basis) showed a deficit of 2 percent of GDP instead of the programmed deficit of 1.4 percent (Table $2 \mathrm{~A}$ ). Net credit to the government, before adjustment for higher-than-projected net external non-project disbursements, was 0.8 percent of GDP higher than programmed.

4. The 2004 budget, presented in accordance with the new economic, administrative, and functional classification, was adopted by Parliament in March 2004. In addition, all special budgets (budgets pour ordre) have been eliminated, although their receipts must still be deposited into the Treasury General Account. The 2004 budget approved by Parliament incorporates the broad aggregates agreed with IMF staff; however, the composition of current expenditure is slightly different and reflects the wage increases negotiated with the unions in the first quarter of 2004. As a result, the budget includes a civil service wage increase of 20 percent (instead of the originally programmed 10 percent), applicable to retirees as well, and 30 percent for primary and secondary school teachers with grade adjustment, totaling 0.4 percent of GDP. The increases took effect on April 1, 2004 (instead of January 1, as originally planned), along with the doubling of transportation allowances in the cities of Kinshasa and Lubumbashi (0.2 percent of GDP). We have maintained the level of primary expenditure of the initial budget by cutting back on official travel, transportation costs, and centralized expenditure (while ensuring that services rendered are paid for promptly). The 2003 budget tracking statements were prepared in accordance with the new classification. This budgetary tool, which considerably strengthens the monitoring of budget execution, will be fully used in 2004.

5. In the first three months of 2004, and despite the civil service strike, revenue (excluding external grants) exceeded program targets by approximately 0.3 percent of GDP, reflecting the supplementary measures (collection of taxes due in the fourth quarter of 2003 and elimination of unjustified exemptions for petroleum products and foodstuffs) implemented since February 2004 to make up for shortfalls in the final quarter of 2003. Total expenditure was 1.1 percent of GDP lower than projected, particularly as a result of the lower level of externally financed investment expenditure. Current primary expenditure was 0.1 percent of GDP lower than programmed, despite a slight increase in the operating expenses of institutions and ministries. Wage expenditure increased less than expected, 
chiefly as a result of the postponement of the civil service wage increase originally scheduled for January 1, 2004. There were no wage payments arrears at end-March 2004. Pro-poor expenditure in the first quarter of 2004 amounted to 0.3 percent of GDP, reflecting the slower disbursement of external financing. Consequently, the domestic primary balance on a cash basis shows a surplus of 0.7 percent of GDP instead of the programmed 0.3 percent, and the consolidated general balance (cash basis) a surplus of 1 percent of GDP compared with the programmed deficit of 0.7 percent. The decrease in net bank credit to the government (0.4 percent) was higher than expected ( 0.2 percent of GDP).

6. The implementation of tax reforms and measures was delayed because of red tape and work interruptions caused by the civil service strike. However, treasury cash management was improved through the introduction of the commitment plan that adjusts on a weekly basis the spending limits allocated to each ministry.

7. Nonetheless, the reforms undertaken to modernize the tax and customs administrations have progressed well. In particular, the General Directorate of Taxes (DGI) has adopted its modernization plan, covering the entire country. Within the DGI, the Large Taxpayers Unit (LTU) has adopted a criterion for the selection of enterprises, and the revision of the new threshold, set at an annual turnover of US\$1 million, will be implemented by end-June 2004. The LTU has now the requisite operational autonomy, including for its audit operations. The draft laws and regulations, needed for the establishment of the tax office for medium-sized enterprises, scheduled for July 1, 2004, have been prepared and will be submitted to Parliament by mid-June 2004. The decision to assign 1,500 Ministry of Finance employees to the DGI, which is contrary to its reform plan, will be repealed by midJune 2004. The Customs and Excise Office (OFIDA) is continuing its reform and modernization program, and the time required for the release of goods at the one-stop window in the port of Matadi has been reduced as a result of the selective inspection of goods and the use of computerized customs procedures. Uniform implementation of the tax laws continues in Boma and in the reunified provinces, where the texts of the laws currently in force have been made available to the administrations concerned. Initially scheduled for endMarch 2004, the new customs code is now expected to be adopted by the government and submitted to the National Assembly in June 2004. The unjustified exemptions for petroleum products and foodstuffs have been eliminated and the computerization of the East Kinshasa offices is under way, following the purchase of computer equipment. Finally, the new harmonized revenue classification for the General Directorate of Administrative and State Revenues (DGRAD) was adopted by Parliament in May 2004 and interministerial decrees establishing the applicable rates are being prepared.

8. The delayed adoption by the National Assembly of the tax policy measures envisaged for 2003 has slightly weakened the expected effects of the tariff and tax reform. Accordingly, the implementing legislation for the March 2003 reform and the necessary adjustments to the legislation itself (eliminating excise taxes on sugar, cement, and matches; no longer limiting the credit mechanism to inputs consumed, and eliminating the turnover tax on exports) were adopted by the government in November 2003 and by the National Assembly only in 
May 2004. The legislative provisions authorizing the Minister of Finance to change the turnover tax rates by ministerial decree and the recent reduction of the turnover tax on services from 18 percent to 13 percent were repealed in June 2004.

9. On the expenditure side, and as envisaged in the program, the government has continued to focus on (a) the strengthening of budget management, (b) a more transparent budgetary process, and (c) enhancement of the capacity to monitor expenditure execution, with special emphasis on pro-poor expenditure.

10. Accordingly, efforts undertaken in the area of public expenditure management have continued. As a result, the budget is now presented in accordance with the new classification and the budget execution process is now functioning, following validation of the new expenditure procedures, from commitment to payment (with the return of BCC debit statements). All expenditure items not channeled through the new expenditure procedures in the first three months of 2004 have been reincorporated. Expenditure items for the month of April not processed using the new budget execution procedures will be reincorporated by end-June 2004. Starting in June, the BCC will transmit its records (journal des opérations) to the Treasury no later than the fifth day of each month. Implementation of three protocols concerning the payroll, public debt transactions, and external disbursements will ensure that, from early June 2004, all expenditures are indeed processed using the new expenditure procedures. The security of the computer link between the BCC and the Treasury, designed to speed up and enhance the reliability of the data exchange between these two institutions, was increased with the installation of a firewall by the BCC. The executions of the 2001 and 2002 budgets were audited by the General Accounting Office (Cour des comptes) and the execution of the 2003 budget will be submitted by end-August 2004. The agreement with the BCC concerning its function of government cashier was adopted by the government and promulgated in April 2004. Finally, work on a double-entry accounting framework for the government, which incorporates the comments of the IMF technical assistance mission, has begun and the simplified accounting framework will be adopted by end-June 2004, as planned.

11. Civil service reform has progressed more slowly than anticipated. The civil service payroll system was audited as planned in April 2004 with assistance from France. However, the schedule for preparing the complete census of government employees could not be adhered to because of a delay in external financing, which only became available in December 2003.

12. The first phase of the retirement program had to be postponed. This phase involves the payment of severance pay to approximately 10,000 employees. The payment, scheduled for April 2004, could not be made as program preparation and the mobilization of financial assistance from the World Bank and the African Development Bank (AfDB) have taken longer than anticipated. 


\section{Monetary policy}

13. In the area of monetary policy, the BCC has gradually decreased its refinancing rate to take account of the slowdown in inflation. Thus, the rediscount rate moved from 20 percent at September 30, 2003 to 8 percent at end-March 2004, whereas the interbank market rate fell from approximately 20 percent to 8 percent over the same period. Banks' average lending rates have also dropped substantially, from 50 percent to 25 percent. With the resumption of growth, private sector credit applications to commercial banks have increased. The stock of billets de trésorerie has remained relatively small.

14. Based on the monetary survey as at end-December 2003, broad money increased by 33 percent in 2003 compared with the programmed 31 percent. By end-March 2004 it had increased by another 10.7 percent relative to end-December 2003, against the programmed 6.3 percent. The velocity of circulation continued to decline, reflecting stronger than programmed demand for money. However, the share of currency in circulation in Congo francs continued to decline in favor of quasi-money and, in particular, of foreign currency deposits. Indeed, the increase in the demand for money denominated in Congo francs in the wake of lower inflationary expectations, banknote shortages in the reunified provinces, and the stability of the Congo franc vis-à-vis the U.S. dollar, was not always met despite the issuance of new CGF 200 and CGF 500 banknotes. The BCC's belated and relatively small purchases of foreign exchange on the market have contributed to the nonobservance of the performance criterion on its net foreign assets at end-March 2004. As a result, the net foreign assets (NFA) of the BCC shrank by US\$82 million at end-December 2003, compared with a programmed contraction of US\$9 million. Owing to delays in disbursements by the AfDB and the World Bank, NFA decreased by US\$2 million at end-March 2004, compared with end-December 2003, against a targeted increase of US\$25 million. Conversely, the net foreign assets of commercial banks grew much faster than expected, by US\$42 million at end-December 2003, and another US\$53 million in the first quarter of 2004. The counterpart of the growth in money supply in 2003 showed a decline in the share of net foreign assets (owing to the decrease in the NFA of the BCC) and an increase in net credit to the government and credit to the private sector (with over 90 percent of the latter in foreign currency). By end-March 2004, this trend had been reversed, reflecting the good fiscal performance, with the increase in net foreign assets and credit to the private sector and a sharp decrease in net credit to the government.

15. The BCC has continued resolutely with the implementation of its action plan aimed at strengthening its institutional and management capacities (Table 3). To respond more fully to the demand for money denominated in Congo francs, the BCC, with the assistance of an IMF resident expert and the National Bank of Belgium (BNB), is conducting a study on the structure of banknote denominations, to be completed by end-July 2004. Also with BNB assistance, the BCC has undertaken a study on its recapitalization. Actual recapitalization cannot occur until the audit ("unqualified opinion") of its December 2003 accounts is completed, which is expected by end-June 2004. Finally, the BCC has drawn up a plan for 
the redeployment of its offices throughout the country. The plan includes the renovation of existing offices and utilization of commercial bank branches already on site.

16. Good progress has been made with the restructuring of the banking system. Based on the audits that were completed in 2003, all banks submitted their restructuring plans to the BCC in January 2004. On this basis, the BCC drew up a list of banks to be liquidated or restructured. Taking account of the seven banks already in liquidation, the BCC has decided to liquidate two additional banks (Banque de commerce et de développement (BCD) and the First Banking Corporation Congo). The restructuring plans for seven other banks have been finalized and approved by the BCC, which has instructed them to begin implementation. Regarding the two additional banks to be liquidated, a call for bids will be launched to select a liquidator by end-June 2004. The social plan for the three public banks in liquidation (BCCE, BCA, and NBK) has been delayed but is expected to be finalized by endSeptember 2004 with World Bank financing. The costs associated with the restructuring of private banks, to be financed domestically, have been included in the 2004 supplementary budget.

\section{Balance of payments and external debt}

17. The external current account for 2003 (including grants) showed a surplus of 0.6 percent of GDP instead of the programmed 2 percent deficit, thanks to the stronger growth of exports and external grants and despite the sharp rise in imports (including unofficial imports). However, this improvement was more than offset by an increase in the errors and omissions item and smaller-than-expected disbursements of project loan financing. Consequently, the overall balance of payments position showed a deficit of US $\$ 348$ million instead of the projected US\$263 million, which was financed by a larger-than-anticipated consolidation of external debt service and a decrease in the banking system's net foreign reserves. The gross foreign reserves of the BCC increased by only US\$22 million instead of the programmed US\$82 million, whereas those of the commercial banks rose by US\$84 million.

18. In the context of the enhanced HIPC Initiative, the government has signed bilateral agreements with all its Paris Club member creditors, with the exception of Japan, Sweden, and the United States. The government is in contact with bilateral creditors with a view to concluding similar agreements. Seven Paris Club creditors have indicated that they will cancel the DRC's debt at the completion point (while Switzerland has already canceled the stock of debt). In addition, four creditors have allowed the suspension of debt service during the interim period. The government has also signed agreements with thirteen commercial creditors. The DRC has honored its external debt service obligations and has accumulated no payment arrears, except with regard to creditors with whom a rescheduling agreement has not yet been signed.

19. In the area of trade policy, the government has eliminated the temporary quantitative restrictions that were set up to address the dumping of certain textile products (printed 
fabrics). In addition, in March 2004 the government concluded an agreement in principle with Zimbabwe to liberalize the exchange rate (which had led to a multiple currency practice contrary to Article VIII, sections 2(a), 3, and 4 of the IMF Articles of Agreement) used for the settlement of commercial transactions between the two countries. Regarding the existing exchange restriction related to the DRC's net debit balance vis-à-vis the other members of the Economic Community of the Great Lakes Countries (CEPGL), the government has contacted these members and will work toward removing this one remaining restriction.

\section{Structural and sectoral reforms}

\section{Anticorruption initiatives, the judiciary, and procurement}

20. Anticorruption initiatives. The draft law against money laundering and the financing of terrorism has been submitted to the National Assembly, and the anticorruption law will be presented by end-June 2004. These two draft laws reflect comments made by the IMF and World Bank staffs. The information campaign concerning the Code of Ethics and Good Conduct for Civil Servants adopted in November 2002 was launched in March 2003 in Mbuji Mayi, but nationwide implementation has been delayed for lack of financing. The same is true of the citizens' vade mecum (compendium of citizens' rights and obligations).

21. Judiciary system. The audit of the entire judicial system began in October 2003 with support from the European Union and other donors. The purpose of this audit, for which missions were sent into the provinces, is to take stock of the judicial system and propose a plan for its strengthening. A preliminary report has already been completed.

22. Procurement. The report on procurement procedures (CPAR), which includes a draft action plan, was submitted to the government at end-April 2004. The recommendations are aimed essentially at (a) the creation of an authority responsible for guiding and implementing reforms (interministerial committee and technical secretariat); (b) the establishment of a temporary legislative and regulatory framework and the recruitment of international experts to strengthen the capacity of the procurement agencies; and (c) the preparation and implementation of large-scale legislative, regulatory, and institutional reforms.

\section{Private sector development}

23. Taxation. In 2003, the government temporarily suspended the imposition of a number of levies, pending the preparation, with World Bank assistance, of a comprehensive action plan for the reform of taxation and quasi-taxation of enterprises. Work is scheduled to begin — with the help of an expert — in July 2004. In addition, the Ministry of the Interior has submitted to the Political, Defense, and Security Commission a draft decree establishing an operational unit responsible for combating police, administrative, and judicial harassment (COLT). The completion of this work will pave the way for the preparation of a comprehensive action plan for the reform of taxation and quasi-taxation of enterprises. Implementation, originally scheduled for July 2004, will be delayed by a few months. 
24. Labor regulation. In April 2004, the National Labor Board adopted 5 of the planned 20 decrees implementing the new Labor Code. Another meeting will be held shortly to continue examining the remaining decrees, to facilitate full implementation of the new Labor Code.

25. Judiciary. With World Bank assistance, training has been provided for approximately thirty judges, assistant judges (assesseurs), and court clerks. The first commercial courts can be established once the buildings are ready.

26. Domestic debt. The cross-arrears between the government and the private sector have been audited. The total amount of certified arrears is US\$840 million. Based on these audits, the Interministerial Committee on Domestic Debt, created by decree in January 2004, continues to work toward reaching agreement between private creditors and the government on the amount, terms, and repayment conditions, taking into account the available financing from the World Bank. An international invitation to bid for the recruitment of a negotiator to help the Commission with its work will be launched by mid-June 2004.

\section{Reform of public enterprises}

27. The Steering Committee on the Reform of Public Enterprises (COPIREP) is now operational. It is responsible for procurement in its areas of competence. The two draft laws on the modernization of the legal framework of public enterprises and on the course that the government's divestment of public enterprises could take, as well as those governing the partnership between public enterprises and the government, are being finalized. The audit of the Public Enterprise Council (Conseil Supérieur du Portefeuille) started slightly behind schedule.

28. The Sectoral Working Groups (GST), created in March 2004, are in the process of defining their sectoral strategies, as well as the restructuring plans for public enterprises in the priority sectors (transport, mines, energy, postal services and telecommunications, and insurance).

\section{Mining sector}

29. In the mining sector, the government has continued to focus on (a) reform of the regulatory framework; (b) the deepening of institutional reforms, notably of the Mining Registry and the Commission for the Validation of Mining Titles; (c) the restructuring of GECAMINES; (d) regulation of the diamond sector; and (e) the certification of mining sector exports and tax revenues.

30. Reform of the legal and regulatory framework of the mining sector. Efforts have focused on the distribution and dissemination of the Mining Code and its implementing provisions. Accordingly, two training seminars for provincial division chiefs and mining operators were held in March in Lubumbashi and Kinshasa. The Mining Code and the Mining Regulation have been distributed to provincial governors and district commissioners, 
who are responsible for their dissemination to associations of mining sector operators and to embassies for dissemination to foreign investors.

31. Institutional reform. Unfortunately, the Mining Registry (CAMI), the key administrative institution responsible for managing the sector's new legal and regulatory framework, and, in particular, the portfolio of mining titles, including collection of the corresponding area taxes, has continued to experience management problems that have hindered the effective start of its operations. Over the next three months, the main responsibility of the new acting director of CAMI is to regularize mining titles and the related administrative files. The Commission for the Validation of Mining Titles, created by decree in March 2003, is not yet operational because the representatives of certain key administrations, including the Office of the President of the Republic, have not yet been appointed.

32. Restructuring of the GECAMINES mining company. The objective is to split GECAMINES into two companies within a reasonable period of time. The new company will receive the productive assets and partnership agreements, whereas the existing company will keep the remaining assets and sell them to settle as many of its liabilities as possible. Substantial progress has been made in this area: the Ministry of Mines has examined the partnership agreements and forwarded its report to the government. However, the hiring of experts (to perform legal, technical, and financial assessments of these partnership agreements and prepare the by-laws of the new company) has been delayed.

33. Regulation of the diamond sector. Diamonds account for more than 60 percent of the country's exports. To improve the regulation of diamond exports, we joined the Kimberley Process. For two years now, the certification of diamonds has been handled by the Ministry of Mines and the Center for Analysis and Evaluation of Precious and Semi-Precious Minerals (CEEC). The Minister of Mines created a Support Committee in August 2003 to implement and monitor the Kimberley Process. The Committee is responsible for (a) ensuring the conformity of national practices with international standards; (b) traceability in the diamond sector, from mine to export; (c) control of the certification of export diamonds; and (d) implementation by the DRC of all provisions required by the Kimberley Process. Our participation in the Kimberley Process is beginning to yield convincing results in the fight against fraudulent exports, particularly between the DRC and the Republic of Congo.

\section{Forestry sector}

34. Forestry reform - and in particular, the new Forestry Code - is intended to (a) enhance the transparency and fairness of access to forest resources, and (b) promote durable development of the sector through sustainable exploitation of forestry resources.

35. The decree of March 2004 calls for the gradual reduction of ONATRA rates during 2004-06 (from US\$16 per cubic meter in 2004 to US\$10 in 2005 and US\$5 in 2006) and gradually increasing the area tax during 2004-07 (from US\$0.0625 per hectare in 2003 to 
US $\$ 0.10$ in 2004, US\$0.20 in 2005, US\$0.30 in 2006, and US\$0.50 in 2007). DGRAD collected the area tax at the rate of US\$0.0625 per hectare in 2003. The April 2004 report on collection indicates that 44 companies have failed to pay a total of US\$506,113. This implies that nearly 7 million hectares of concessions are still allocated to holders who are incapable of honoring the contracts negotiated with the government. The report on collection has been forwarded to the Ministry of Environment for cancellation of the noncomplying contracts, pursuant to Article 8 of the Forestry Code.

36. Conversely, the other reforms we intended to undertake were delayed and had to be postponed, particularly the implementation of the Program to Secure Forestry Revenue, the transfer of 40 percent of the area tax receipts to local governments, the preparation and adoption of regulations implementing the Forestry Code (awarding of concessions, exploitation and development, and combating illegal operations), and the submission to Parliament of the draft Law on the Conservation of Nature. Moreover, contrary to the moratorium on such contracts, in early 2003 concessions covering 6-9 million hectares were allocated by mutual agreement (allocation de gré à gré), and certain contracts that had been canceled in April 2002 were reinstated, by way of exception, in early 2004.

\section{Health sector}

37. The health sector strategy will be prepared with World Bank assistance and based on the findings of the Poverty/Health Status Report (RESP). A preliminary report was presented to the donors' round table in May 2004. Implementation of the eight contracts for health infrastructure rehabilitation signed with the authorities (Maîtres d'Ouvrage Délégués $M O D)$ is progressing satisfactorily.

\section{Education sector}

38. Preparation of the overall framework for development of the education sector will be based on the Status Report on the National Education System (RESEN). The preliminary version of this report was finalized and discussed in a workshop in March 2004. The refurbishment of 126 schools - financed by the World Bank in the provinces of Kinshasa, Bas-Congo, Kasaï Occidental, and Katanga - is progressing far more slowly than expected (40 schools have been renovated to date) because of procurement delays and capacity constraints of suppliers.

\section{Program for the demobilization and reintegration of ex-combatants}

39. The government has defined a National Disarmament, Demobilization, and Reintegration Program (DDR) for Congolese armed groups that signed the Lusaka Agreement. The program comprises three components: (i) demobilization and reintegration of combatants from the above-mentioned groups, estimated at between 150,000 and 200,000 individuals; (ii) assistance for the socioeconomic reintegration of ex-combatants; and (iii) special aid for vulnerable groups. The national DDR program is to be financed primarily by an IDA grant of US\$100 million and a grant from the Multi-Country Demobilization and 
Reintegration Program (MDRP), also in the amount of US\$100 million. The World Bank has agreed to help the DRC seek additional financing under the MDRP, if necessary.

40. The government has defined the institutional framework for the DDR in consultation with donors and lenders. This framework consists of (i) the National DDR Commission, responsible for developing strategic guidelines, and (ii) the Executive Secretariat, responsible for implementing those guidelines. The institutional framework of the DDR, established by two presidential decrees, includes (i) the Interministerial Committee for the DDR, responsible for overseeing DDR activities at the national level; (ii) the National Commission for the DDR (CONADER), created by decree in December 2003, which will coordinate the preparation and implementation of the national program; and (iii) the Committee for the Management of Financial Resources and the Coordination of DDR Financing (CGFDR) of December 2003. The implementing decrees are being prepared. The government has appointed the General Coordinator and Deputy General Coordinator of CONADER.

\section{Poverty reduction strategy}

41. All activities programmed since October 2003 to prepare for the launch of the process of formulating the full Poverty Reduction Strategy Paper (PRSP) by end-October 2005 have been completed. These include (a) launch in mid-December 2003 of the information, education, and communication campaign (IEC) on the process of formulating the full PRSP, currently under way in the media; (b) organization in January 2004 of the workshop to launch the PRSP formulation process; (c) finalization, in an international seminar held in Kinshasa in January 2004, of the questionnaire and the methodology for the participatory consultations to be organized at all levels (national, provincial, local, and community-based), which led to the selection of the participatory methods to be applied and the poverty survey; and (d) the appointment of provincial and local committees, as well as sectoral focal points.

42. In addition, by April 2004, preparations had ben made for participatory consultations, units responsible for participatory consultations had been selected, and four experts were hired to enhance the operational capacities of the PRSP Steering Group. The PRSP Technical Committee has been expanded to 25 members, and in March 2004 the terms of reference for the thematic and sectoral consultations were finalized.

43. The 1-2-3 poverty survey was launched in May 2004 with the support of the National Capacity Building Secretariat (SENAREC), AFRISTAT, and the African Development Bank. The survey is being conducted initially in Kinshasa (326 districts) before being extended to the other provinces. The establishment of mechanisms for the management of the Trust Fund for PRSP formulation was delayed because of donors' insistence that it be managed in accordance with the procedures suggested by the World Bank. However, following the preparation of the procedures manual and the hiring of an administrative and financial manager, the mechanisms were put in place in May 2004. The Trust Fund Management Unit is also operational. Participatory consultations are expected to begin in June 2004. The terms of reference and structure of the committee responsible for monitoring poverty reduction 
expenditure will be modified to increase its independence and enable it to exercise greater expost control of the use of resources intended to finance pro-poor expenditure.

\section{Policies ANd Measures for 2004}

44. Consistent with the medium-term economic framework, which has been revised to take account of the updated estimates of the impact of the changing international environment, the preparations for the 2005 elections, the implementation of our national DDR plan, the successful reunification of our country, and the servicing of our external debt, we have finalized the following objectives for the year, which remain broadly in line with the preliminary objectives approved at the time of the third review of our program with Fund staff: (a) an economic growth rate of 6.3 percent; (b) an average inflation rate of 5 percent; and (c) an external current account deficit (including grants and after external debt service relief) of 2 percent of GDP, associated with the substantial increase in investment financed by international assistance (Table 1). We will take advantage of the obtained external debt service relief to increase the share of pro-poor spending in our budget and to strengthen the international reserves of the $\mathrm{BCC}$, which guarantee the stability of our national currency.

\section{Fiscal policy}

45. Macroeconomic stability will continue to be supported by a prudent fiscal policy, while steps are taken to ensure that the composition of expenditure reflects our poverty reduction objectives described in our interim PRSP. The 2004 budget, adopted by Parliament in March 2004, did not take account of the establishment of our national DDR program, the repayment of domestic debt, the cost of preparing the elections, and the cost (domestically financed) of the liquidation of a number of commercial banks. To take account of the immediate impact of these programs, the government will adopt a supplementary budget in June 2004 that will be prepared in accordance with the new classification and submitted to the National Assembly in July 2004. A preliminary survey of the situation of the revenuecollecting agencies (régies financières) and Treasury branches in all reunified provinces has been prepared. Before the new Law on Decentralization is adopted by Parliament, we plan to carry out a comprehensive study on its financial implications with the help of an IMF technical assistance mission. We will continue to work toward implementation of the tax reform program launched with the help of IMF staff, as well as the enforcement of the tax laws throughout the national territory. We will also continue to make the necessary efforts to ensure that revenue collected in the mining sector and in the reunified provinces is transferred as quickly as possible to the general account of the Treasury.

46. With the pursuit of the revenue-generating measures introduced in the first quarter of 2004, the projected increase in the producer prices of oil, and improved expenditure control, especially through strict monitoring of the cash-flow plan, total revenue (excluding external grants) for 2004 is expected to reach 9.2 percent of GDP, while total expenditure should increase by 2 percent of GDP as compared to the original program. Pro-poor spending will increase from 1.5 percent of GDP in 2003 to 6.6 percent in 2004, while security spending 
will remain at 2.1 percent. The consolidated domestic primary balance on a cash basis is expected to show a surplus of 1 percent of GDP, and the overall consolidated balance, including the net balance of BCC operations, is expected to show a cash-basis deficit of 3.2 percent of GDP (Table 2B). It is anticipated that net credit to the government will be negative by an amount equivalent to 0.6 percent of GDP.

47. On the revenue side, reforms will be pursued with a view to introducing a valueadded tax (VAT) in 2006. Efforts will continue, with IMF technical assistance, to increase the effectiveness of the administrations responsible for revenue collection. A plan for the redeployment of the revenue-collecting agencies throughout the country is being formulated, and the government is seeking support from the international community to strengthen the administrative capacity of these agencies at the provincial level. Within this framework, the revenue-collecting agencies will maintain their current operating procedures, and oil production revenue will not be included in the calculation of the amount (rétrocession) to be transferred to them. In addition, the concept of the fiscal franc (franc fiscal) will no longer be used by the revenue-collecting agencies. The DGI will pursue its reform plan, ensuring that (a) the three draft laws and the draft regulatory decree necessary for the establishment of the tax office for medium-sized enterprises are adopted by the government by mid-June 2004; and (b) the computer link between the LTU and the BCC become fully operational in early August. With respect to customs, OFIDA will also continue with its modernization program, in particular with the computerization of the East Kinshasa customs office by endSeptember 2004. A memorandum of understanding between the Congolese Control Office (OCC) and OFIDA on the harmonization of the valuation of imports will be signed by endJuly 2004.

48. In addition, the following measures will be introduced to increase revenue mobilization: (a) maintenance of the turnover tax rate on services at 18 percent; (b) revision of the tax agreement (convention) between MIBA and the government by endSeptember 2004; (c) adoption by the government of the draft laws necessary for the establishment of the tax office for medium-sized enterprises, scheduled for July 1, 2004; (d) adoption by the government of the regulations confining turnover tax deductibility to large enterprises by end-July 2004; (e) finalization of the complete inventory of tax exemptions granted by the Ministry of Finance and the oversight ministries, as well as their rationalization, by end-September 2004; (f) completion by the Ministry of Finance of the audit of the principal entities receiving duties and fees not collected by the DGRAD (OCC, ONATRA, OGEFREM) by end-December 2004; (g) review of the taxes on money transfers to prevent double taxation, by end-June 2004; and (h) application of the tax sticker (vignette) on tobacco throughout the country, as of end-June 2004.

49. In the 2004 supplementary budget, the increase in the wage bill covers the following: (a) the salary increases and the doubling of transportation allowances provided for in the 2004 budget; (b) the later-than-planned demobilization of former combatants - this operation will not begin until October 2004 and will affect only 50,000 soldiers out of the estimated total of 150,000; and (c) the retirement of 10,000 employees during the last quarter 
of 2004, with World Bank assistance. The civil service reform will be launched, with priority given to (a) the implementation of the short-term recommendations of the payroll system audit (assignment of payment officers to the Treasury, return of initialed payrolls to the payment officers, and adequate budgetary control of the use of resources); (b) the continued elimination of ghost workers from the payroll, with the validation of personnel lists by all Ministers and officials responsible for affected budgets annexes, cross-checking of the lists by the Ministry of Civil Service, and verification by the Payroll Directorate in the Ministry of Budget by end-July 2004; and (c) the selection of consultants in September 2004 to launch the census of government employees throughout the country, the results of which are expected by end-April 2005. There will then be another screening of the staff lists; personnel and payroll files will be created; and a list of employees eligible for the retirement program will be drawn up. The second phase of this retirement program, affecting some 62,000 employees, will occur only during the second half of 2005.

50. The control of operating expenditures will improve, with strict observance of the cash-flow plan and special attention paid to official travel, transportation costs, and aircraft charters. For centralized expenditures, a lump sum, financed with World Bank assistance, was negotiated with SNEL and REGIDESO for electricity and water consumption in 2004.

51. The government undertakes to pursue resolutely the reforms aimed at increasing the transparency of fiscal management. Budget tracking statements (ESBs) will be available no later than the tenth day of the following month, starting with those for July 2004. These ESBs will include (a) expenditure resulting from the three protocols (payroll, public debt, and external resources); (b) all expenditure that by-passed the expenditure procedures; and (c) specific pro-poor spending. In addition, the following measures will be implemented in 2004: (a) adoption in September 2004 of regulations governing the eligibility and management of the budgets annexes, with a view to their rationalization in the preparation of the 2005 budget; and (b) adoption by the government of the criteria developed by the Treasury and Payment Authorization Directorate for the opening of government accounts at the BCC and at commercial banks, and the closing of unauthorized bank accounts by end-December 2004. The work related to the implementation of a double-entry accounting framework for the government is under way, and a schedule has been drawn up for the introduction of the simplified framework by January 1, 2005 and the implementation of the government chart of accounts by January 2006.

52. Pending finalization of the study on the financial implications of the new Law on Decentralization submitted to Parliament, the government has decided to respect the following principles: (a) provincial budgets must be balanced; (b) decentralized government entities (EADs) will not contract external debt without the prior approval of the Minister of Finance and the prior consent of the BCC and OGEDEP; (c) EADs will not contract domestic debt; (d) EADs will be unable to create new taxes without the approval of the Minister of Finance; (e) revenue-collecting agencies - Customs in particular-will operate solely under the authority of the central government; and (f) the current level of transfers to the provinces will be maintained, while any increase in transfers will be accompanied by corresponding 
spending authority. Each provincial governor will prepare a diagnostic study of his province and a roadmap of measures for implementation. A list of technical assistance needs and priorities to strengthen administrative capacity in the provinces will be drawn up. In this context, the government will request assistance from the international community to strengthen fiscal management in the provinces, starting with the province of Kinshasa.

\section{Monetary policy}

53. The BCC will pursue a monetary policy that aims at price stability in the context of the floating exchange rate system. To this end, broad money is expected to increase by 29 percent (Table 1), a rate exceeding that of nominal GDP growth, to take account of the increase in the demand for money. Net banking system credit to the government will decrease by 14 percent of the beginning-of-period money stock, which will make it possible to satisfy the upswing in private sector demand for credit and boost the net foreign assets of the BCC to US\$-598 million. Also, gross foreign assets will grow from three weeks of imports at end2003 to more than six weeks at end-2004.

54. The BCC plans to adopt a much more active policy of net foreign exchange purchases on the exchange market. Without running counter to economic fundamentals, this approach will enable the BCC to consolidate its international reserves while at the same time responding to the increased demand for Congo franc. In addition, the BCC will ensure strict enforcement of the requirement to surrender export earnings after 60 days, as prescribed in the legislation.

55. The government undertakes to safeguard the full independence of the BCC in the pursuit of monetary policy, in particular its interventions on the exchange market and the issuance of higher denomination Congo franc banknotes. It is expected that the study undertaken with BNB assistance on the volume and structure of Congo franc banknotes will be completed by end-July 2004. Issues of new banknote denominations will be prepared carefully and preceded by a public awareness campaign, as was the case for the highly successful introduction of CGF 200 and CGF 500 banknotes. The BCC will continue to strengthen its monetary policy management, in particular by streamlining its financing instruments while limiting the risk of its interventions. To this end, a new instruction on refinancing operations and related collateral will be adopted by end-July 2004. Lastly, the monetary authorities undertake to ensure transparency in their operations and functioning, in conformity with the IMF Code of Good Practices on Transparency in Monetary and Financial Policies.

56. The BCC will continue its restructuring through the implementation of its action plan prepared with Fund staff. The improvement of the BCC computer system remains a key component of the action plan. Guided by the Information Technology (IT) Committee established in April 2004, the BCC will select appropriate computer applications, especially in the areas of accounting and foreign exchange transactions, and will launch a call for bids by end-September 2004. A commission of independent experts will be appointed to improve 
the monitoring and control of IT purchases. In addition, the BCC will adopt its IT sector plan by end-September 2004. This restructuring is primarily intended to modernize the BCC's infrastructure, as well as its operational systems and procedures, having in mind the conduct of monetary policy and control and supervision of the banking system. To achieve this, the $\mathrm{BCC}$, assisted by the BNB, will also complete a study on its recapitalization by endSeptember 2004. This study will be based on the ongoing external audit of the BCC financial accounts at end-2003 and on the consolidation of its operating account through a series of specific actions aimed at improving its revenues and better controlling its expenditure. Nevertheless, it will take some time before the financial position of the BCC improves because of the financial disintermediation in the country. In the short term, this restructuring and the redeployment of BCC agencies throughout the country are causing costs that will continue to be covered by the government budget - the government being the sole shareholder of the BCC - and external financing that is being sought. The BCC is implementing a plan for the redeployment of its agencies throughout the country, which it intends to present at a donors' meeting at end-2004.

57. The BCC will strictly implement its cash-flow plan, whose deficit for 2004 is estimated at 0.6 percent of GDP. In this context, it will strictly limit official travel and stress transparency, good practices in the conduct of its business, and anticorruption efforts at all levels. The principle of transparent bidding procedures will be strictly observed, and the responsible committee will be enlarged to ensure better preparation of projects and greater transparency, including in ex-post control and monitoring.

58. Banking supervision. The BCC will continue to strengthen banking supervision with the assistance of Fund staff. This involves, in particular, (i) the computerization of supervision (to be completed by end-December 2004), which will facilitate improved off-site supervision; (ii) introduction of the plan for the verification of on-site inspections by endSeptember 2004; and (iii) adoption of the new chart of accounts for commercial banks and the electronic transmission of periodic statements by banks, starting in August 2004. In the area of microfinance, the BCC will conduct a survey of microfinance institutions and draft a new law to replace the existing instruction by end-December 2004.

59. The BCC will closely monitor the restructuring of the banking system, which is essential for financial reintermediation. It will also supervise the redeployment of the commercial banks in the provinces. It will improve the content of the monetary statistics and their dissemination to banks, as well as the risk control statistics. The government is committed to taking all necessary steps to ensure that the social plans of the three public commercial banks in liquidation are quickly completed and result in actual payments to the laid-off staff (by end-December 2004), financed by the World Bank. Finally, the government has launched a reform of the tax and legal framework for commercial banks to make it more transparent and more in line with good international practice. The decree amending the chart of accounts to facilitate the reconstitution of equity capital was adopted in early 2004. In addition, draft laws introducing tax deductibility of statutory loan-loss provisions, the 
reduction of the personal property tax (from 6 percent to 1 percent), and a more attractive investment regime for the financial sector will be completed by end-December 2004.

\section{Structural and sectoral reforms}

\section{Anticorruption initiatives, the judiciary, and procurement}

60. Anticorruption efforts will be expanded to cover the entire country and all decentralized government entities and public enterprises. The action plan on procurement reform is expected to be adopted by end-October 2004, concurrently with the appointment of officials responsible for its implementation. Meanwhile, to improve transparency, the government will ensure strict enforcement of the procedures manual, in particular through the use of bidding procedures for any contract valued at more than US\$10,000. It is anticipated that the final version of the action plan to strengthen the judiciary will be available by endJune 2004 and implemented by end-December 2004.

\section{Private sector development}

61. Taxation. The action plan for reform of the tax regime applicable to enterprises is expected to be completed by end-December 2004. Preparation of the implementing decrees for the Labor Code will continue, and on the legal side, the government, with the assistance of external consultants, will continue the process of accession to the OHADA Treaty (including the adoption of the national legislation to the OHADA Treaty).

62. Domestic debt. Our efforts will focus on the preparation and implementation of the strategy for the settlement of net cross-arrears between the government and the private sector. Agreement on this, expected by end-2004, will establish a discount for each type of creditor. An invitation to bid will be launched to select a commercial bank to make the payments once the discounts have been agreed with creditors representing 75 percent of the amount of the cross-arrears. The repayment plan, which is included in the draft supplementary budget for 2004, will be implemented with World Bank financing.

\section{Reform of public enterprises}

63. Based on the audit of the Public Enterprise Council (Conseil Supérieur du Portefeuille-CSP), it is expected that the plan to restructure the CSP and strengthen its capacity to monitor and supervise public enterprises will be completed by endSeptember 2004. The draft laws on the reform of public enterprises and government divestiture will be adopted by the government for submission to the National Assembly by end-October 2004. The government will adopt the plans to restructure ONATRA, SNEL, Regideso, RVA, Citytrain, SNCC, LAC, and OCPT by end-December 2004. A plan to restructure the oil distribution company (COHYDRO) will be completed by endSeptember 2004 on the basis of a financial audit and the adjustment of its accounts. The program of voluntary separations from OCPT, financed by the World Bank, could be 
completed by end-December 2004, following a six-month delay. The restructuring plan for GECAMINES will be submitted to the National Assembly in 2005.

\section{Mining sector}

64. The Mining Code will be disseminated throughout the country during the second half of 2004, with assistance of experts selected by a call for bids. During the second quarter of 2004, the preparation of the new by-laws of the Mining Registry (CAMI) will be completed, and a call for bids will be launched for the recruitment of its new management team. In addition, an organizational audit and a financial audit, which will serve as the basis for the restructuring of CAMI, will be undertaken. We will ensure that (a) the auditors are selected through a public call for bids by end-August 2004; (b) work will start in early September 2004; and (c) the results will be available by end-December 2004. The results of this audit will be used to establish efficient financial management and supervision procedures, as well as to monitor and assess the performance of CAMI, in the interest of safeguarding the credibility of the new legal framework for the mining sector. Our aim is to complete the restructuring of CAMI by end-March 2005. The Commission for the Validation of Mining Titles is expected to become fully operational by end-June 2004, so that decisions can be made by end-September 2004 regarding 85 percent of the various titles identified.

65. Given the delay in the recruitment of experts, the final restructuring strategy for GECAMINES will be adopted by end-June 2005 and submitted to the National Assembly soon afterwards. However, the government has authorized the hiring of a firm with an excellent international reputation in mining to manage GECAMINES until the new company is formed, with the specific objective of boosting production and protecting the company's equity. The selection of this firm in an international call for bids is under way and is expected to be completed by end-June 2004 .

66. With respect to the diamond sector, we continue to be concerned by the fact that export growth has not led to a subsequent increase in tax revenue, which is still very low. We therefore hope to receive assistance from the international community for formulating a sectoral approach to combating fraud in the diamond sector, starting with audits - by an international firm and in accordance with international accounting standards - of the principal enterprises and institutions in the sector (in particular, MIBA, SENGAMINES, and CEEC), as well as the artisanal sector. The terms of reference are being drawn up, and by end-September 2004 we will launch an international call for bids for an audit financed by the European Union. The net claims of these enterprises on the government will be settled on the same terms as the claims of other creditors, as defined in the overall strategy for the settlement of cross-arrears between the government and the private sector, currently being developed with World Bank assistance.

67. Certification of exports and tax revenue. To minimize the losses of foreign exchange and tax revenue resulting from fraudulent practices, we are introducing procedures to control the quantity, value, and quality of exports in terms of ore grade. In this regard, we 
have awarded a tender to a specialized company to check the grade and weights of exports. We also intend to set up a program to secure mining revenue, similar to the one proposed in the forestry sector. At the same time, the government will establish a one-stop window for mining exports in Katanga, consistent with the provisions of the Mining Code. To that end, the government will, by end-October 2004, organize joint training programs for staff of the Ministry of Mines and the Ministry of Finance on the tax collection procedures described in the new Mining Code. We expect that the one-stop window for mining exports will be operational by end-December 2004.

\section{Forestry sector}

68. In the forestry sector, the following five measures will be taken by endSeptember 2004. First, the government plans to resume strict enforcement of the moratorium on forestry concessions allocated by mutual agreement, pending completion of the process of converting canceled contracts and implementation of the new procedures for awarding concessions. All applications for forestry concessions will be considered within the framework of the conversion process. Second, we will ensure strict enforcement of the new tax regime set out in the interministerial decree of March 2004, particularly the reduction of the ONATRA tariff from US\$16 to US\$10 per cubic meter and the increase in the area tax from US\$0.0625 to US\$0.10 per hectare. In this regard, we plan to create a joint monitoring committee, with participation of all parties involved and donor representatives as observers. Third, by June 2004 we will publish the report on the collection of the area tax for 2003 and enforce the contractual provisions requiring automatic cancellation of the concessions of those companies that have not paid the tax (Article 8 of the Code). Fourth, 40 percent of the area tax collected for 2003 will be transferred to decentralized local entities. Fifth, we will launch the program to convert canceled contracts into "sustainable management concessions", in accordance with the new Forestry Code. This program will be carried out with the help of an independent observer and will be completed within 12 months following its launch. Lapsed or noncomplying contracts will be canceled, and the new list of valid concessions will be published.

69. By March 2005, the government will have introduced the Program to Secure Forestry Revenue, to be managed jointly by the Ministry of Finance and the Ministry of the Environment. It will continue to apply the tax and quasi-tax provided for in the decree of March 2004 (ONATRA tariff reduced to US\$5.00 per cubic meter as of January 1, 2005, and area tax increased to US\$0.20 per hectare). Regulations implementing the new Forestry Code (awarding of concessions, sustainable management, logging, combating illegal operations, and community forestry) will be adopted after consultation with the parties involved. Also, the government will have launched the process of forestry zoning, and to the extent that it wishes to award new concessions, will have first consulted with neighboring villages. Finally, an independent mechanism for the on-site inspection of logging operations and sustainable development will be introduced. Meanwhile, the draft law on the Conservation of Nature will be prepared for adoption by the government by end-2005. 
70. Social sectors. The diagnostic reports on health, education, and welfare currently being prepared will be completed with World Bank support. They will serve as the basis for preparation of the sectoral strategies that are triggers for the HIPC Initiative completion point.

71. Health sector. It is anticipated that the final version of the RESP will be available by end-October 2004. During the period 2004-05, discussions on the regulatory and fiscal reforms for the sector and the preparation of a sectoral project with the help of the World Bank are expected to begin. By September 2004, the accelerated implementation of eight MOD contracts is expected to improve perceptibly the delivery of basic services to the population, especially in the area of infant mortality.

72. Education sector. It is expected that the final Status Report on the National Education System (RESEN) will be available by end-August 2004, following inclusion of the analysis of data from the eastern part of the country, and it will be widely disseminated by end-September 2004. The RESEN report will be a key input for (i) the drafting of the education section of the full PRSP and the preparation, by August 2006, of the sectoral strategy, which, as in the case of the health sector, is a trigger for the enhanced HIPC Initiative completion point; (ii) the finalization of the action plan, now under preparation, for the "Education for All" (Education Pour Tous) program, which we hope to submit to the international community as soon as possible after completion of the RESEN and in any event by end-December 2004; and (iii) the preparation of the World Bank education sector project by March 2005. Lastly, the refurbishing of 126 schools will be completed by endJanuary 2005.

73. In the area of infrastructure rehabilitation, all projects financed by the first tranche of the World Bank Emergency Multisectoral Rehabilitation and Reconstruction Program (EMRRP) will be completed in 2005, and an overall reconstruction and rehabilitation policy will be finalized.

74. Welfare sector. A comprehensive program for the reform of this sector will be developed on the basis of studies carried out in 2002-03. The program could be finalized by end-June 2004, and will include actions to be taken by the government and its partners in the area of welfare, as well as the budget and sources of finance. Financing for activities benefiting "vulnerable groups" will be sought. The project to establish a Social Fund, supported by the World Bank, is expected to begin in the course of 2004 and be completed by end-2005. External financing for an audit of the National Social Security Institute will be actively sought.

75. Program for the demobilization and reintegration of former combatants. By endSeptember 2004, the government is expected to (i) adopt the national DDR program in the Council of Ministers; (ii) finalize the implementing regulations for the decrees signed in December 2003 to establish the National Commission for the DDR (CONADER) and the Committee for the Management of Financial Resources and the Coordination of DDR Financing (CGFDR); (iii) complete the preparation of the joint operating plan that brings 
together the program for the restructuring of the army and the national DDR program; and (iv) appoint the key staff of CONADER. The disarmament and demobilization phase could begin in August 2004 and could continue for 18 months, followed by the reintegration phase, which could begin in September 2004 and last three years.

76. Poverty reduction strategy. The participatory consultations will be carried out in the period June-August 2004. The reports on the sectoral and community consultations will be drafted and forwarded to the technical ministries and communities for feedback in August and September 2004. Based on the consultations and the thematic work, the thematic groups and sectoral ministries will prepare their contributions to the PRSP by end-October 2004. The initial version of the PRSP, without the complete statistical data, will be finalized in December 2004.

77. Concurrently with the qualitative poverty survey to be conducted on a participatory basis, a survey on household consumption, employment, and the informal sector (1-2-3 Survey) is planned for both urban and rural areas and is to be finalized by February 2005. It will be conducted jointly by the Poverty Reduction Strategy Technical Committee (CTSRP) and the National Statistics Institute (INS), to give the latter a pivotal role in the collection, analysis, and dissemination of data on poverty. It is expected that the 1-2-3 Survey will be returned in May 2005 and that it will be incorporated into the second version of the PRSP in June 2005. The full PRSP will be delivered and returned to the country's national and international partners in July and adopted by the government by end-August 2005 .

\section{Statistical apparatus}

78. The government, with assistance from the international community, and in particular with the help of the IMF resident expert who will take up his duties shortly, intends to strengthen its statistical apparatus, specifically with a view to improving public debt management, the national accounts, and the balance of payments. The government will seek the support of the international community to help build administrative capacity for the collection and processing of statistical data within the central government and in all provinces. The governors of the provinces will perform a quick diagnostic analysis of the statistical apparatus of the provinces and identify the immediate measures to be adopted as well as technical assistance needs. In addition, in April 2004 the government joined the IMF’s General Data Dissemination System (GDDS).

\section{Monitoring, Prior Actions, Performance Criteria And Indicators}

79. The Interministerial Committee Responsible for Monitoring Programs Concluded with the Bretton Woods Institutions (CISPI), chaired by the Minister of Finance, and the Interministerial Commission Responsible for Formulation of the Poverty Reduction Strategy, chaired by the Minister of Planning, will continue to implement the measures of the Government Economic Program (PEG) and the poverty reduction strategy. The two interministerial commissions will continue reporting to the Economic and Financial 
Commission, the role of which is to ensure the coordination of all programs. The latter will, in particular, ensure the establishment of the rule of law, including the strict enforcement of the applicable legislation and codes, and will ensure that any new agreement or contract with the government (or guaranteed by it), including public enterprises and the provinces, is consistent with them. The same will also apply to any loan contracted (or guaranteed) by the government (including the decentralized entities (EADs)), the terms of which must respect the concessionality defined in the technical memorandum of understanding. The circular stipulating that any new external borrowing must be approved in advance by the Minister of Finance (with the prior consent of the BCC and OGEDEP) will be widely disseminated among the sectoral ministries and EADs. All new external debt contracted (or guaranteed) by the government, including EADs, will be recorded by OGEDEP. The government's letter of intent and memorandum on economic and financial policies will be broadly disseminated to the concerned units.

80. To ensure the success of the program, the government will implement the following three prior actions: (a) adoption by the government of the 2004 supplementary budget, reflecting the broad aggregates agreed with IMF staff and presented according to the new classification (June 2004); (b) adoption by the government of the new Customs Code (June 2004); and (c) adoption by the government of the Anticorruption Law (June 2004) (Table 5).

81. Program implementation in 2004 will continue to be monitored by means of semiannual reviews, semiannual quantitative performance criteria (September 2004), and quarterly benchmarks (June, September, and December). As shown in Table 4 and defined in the technical memorandum of understanding, this involves (a) a floor on the net foreign assets of the $\mathrm{BCC}$; (b) a ceiling on the net domestic assets of the $\mathrm{BCC}$; (c) a ceiling on net bank credit to the government; (d) a ceiling on BCC credit to nonfinancial public enterprises; (e) a ceiling on $\mathrm{BCC}$ credit to the nonfinancial private sector; (f) a ceiling on new nonconcessional external debt (with a grant element of less than 35 percent) contracted or guaranteed by the government (or EADs) with maturities of more than one year (excluding IMF credit); (g) a ceiling on new nonconcessional external debt with an initial maturity of less than one year (with the exception of normal import credits) contracted or guaranteed by the government; and (h) no accumulation of civil service, police, military, or BCC arrears on wages (including all forms of compensation). The program will include three continuous performance criteria: (a) the BCC will not finance any budgetary expenditure not previously authorized by the Ministry of Finance; (b) the BCC will not purchase domestic and foreign currency banknotes on the market at a premium against bank money payments; and (c) the government will not accumulate external arrears on debt service for which a rescheduling agreement has been concluded with its creditors or on any new borrowing. Finally, the BCC will continuously maintain SDR 8 million in its accounts with the IMF to ensure the regular payment of its obligations to the Fund. 
82. The program also includes a structural performance criterion for end-September 2004 (Table 5), namely, the selection of an international firm to conduct the external audit of MIBA. The structural benchmarks for the period June-December 2004 include the following:

(i) finalization of the COHYDRO reorganization plan (June 2004);

(ii) completion of the strategic audit of the Public Enterprise Council (Conseil Supérieur du Portefeuille) (June 2004);

(iii) adoption of a simplified, double-entry accounting framework for the government (June 2004);

(iv) finalization of the Law Governing Public Institutions and the Law Governing Divestment by the State of Public Enterprises (December 2004); and

(v) reorganization of the procedures for paying civil service servants based on the recommendations of the external audit of the payroll system (December 2004). 
Table 1. Democratic Republic of the Congo: Selected Economic and Financial Indicators, 2002-07

\begin{tabular}{|c|c|c|c|c|c|c|c|c|c|c|c|}
\hline & \multirow[t]{2}{*}{2002} & \multicolumn{2}{|c|}{2003} & \multicolumn{2}{|c|}{2004} & \multicolumn{2}{|c|}{2005} & \multicolumn{2}{|c|}{2006} & \multirow{2}{*}{$\begin{array}{l}2007 \\
\text { Proj. }\end{array}$} & \\
\hline & & $\begin{array}{c}\text { Country } \\
\text { Report No. } \\
04 / 97\end{array}$ & Est. & $\begin{array}{c}\text { Country } \\
\text { Report No. } \\
04 / 97\end{array}$ & Rev. Prog. & $\begin{array}{c}\text { Country } \\
\text { Report No. } \\
04 / 97\end{array}$ & Proj. & $\begin{array}{c}\text { Country } \\
\text { Report No. } \\
04 / 97\end{array}$ & Proj. & & \\
\hline & \multicolumn{10}{|c|}{ (Annual percentage changes) } & \\
\hline \multicolumn{12}{|l|}{ Output and prices } \\
\hline Real GDP & 3.5 & 5.0 & 5.6 & 6.0 & 6.3 & 7.0 & 7.0 & 7.0 & 7.0 & 7.0 & \\
\hline GDP deflator & 32.0 & 12.5 & 12.4 & 5.4 & 5.0 & 5.0 & 5.0 & 4.5 & 5.0 & 5.0 & \\
\hline Consumer prices, annual average & 25.3 & 13.0 & 12.8 & 6.0 & 5.0 & 5.5 & 5.0 & 5.0 & 5.0 & 5.0 & \\
\hline Consumer prices, end-of-period & 15.8 & 4.4 & 4.4 & 6.0 & 6.0 & 5.0 & 5.0 & 5.0 & 5.0 & 5.0 & \\
\hline \multicolumn{12}{|l|}{ External sector } \\
\hline Exports, f.o.b. (in U.S. dollars) & 22.2 & 15.5 & 19.1 & 10.7 & 10.3 & 11.5 & 5.1 & 11.2 & 8.7 & 9.5 & \\
\hline Imports, f.o.b. (in U.S. dollars) & 35.4 & 25.4 & 28.5 & 26.8 & 33.4 & 15.1 & 20.9 & 11.2 & -2.2 & 9.8 & \\
\hline Export volume & 21.9 & 11.5 & 13.6 & 11.7 & 4.3 & 12.5 & 7.0 & 12.2 & 11.5 & 10.5 & \\
\hline Import volume & 30.5 & 18.9 & 19.6 & 24.7 & 23.5 & 13.8 & 22.4 & 10.0 & 1.0 & 10.0 & \\
\hline Terms of trade & 3.9 & -1.7 & -2.4 & -2.5 & -2.1 & -2.0 & -0.6 & -2.0 & 0.6 & -0.7 & \\
\hline Nominal effective exchange rate 1 / & -71.1 & $\ldots$ & -19.5 & $\ldots$ & $\ldots$ & $\ldots$ & $\ldots$ & $\ldots$ & $\ldots$ & $\ldots$ & \\
\hline Real effective exchange rate $1 /$ & -56.2 & $\ldots$ & -11.1 & $\ldots$ & $\ldots$ & $\ldots$ & $\ldots$ & $\ldots$ & $\ldots$ & $\ldots$ & \\
\hline \multirow{2}{*}{\multicolumn{11}{|c|}{ (Annual change in percent of beginning-of-period broad money; unless otherwise indicated) }} & 1 \\
\hline & & & & & & & & & & & $\hat{\omega}$ \\
\hline Broad money & 25.7 & 31.2 & 32.7 & 25.0 & 28.7 & $\ldots$ & $\ldots$ & $\ldots$ & $\ldots$ & $\ldots$ & 1 \\
\hline Net foreign assets & 10.4 & 10.9 & -14.1 & 22.8 & 35.1 & $\ldots$ & $\ldots$ & $\ldots$ & $\ldots$ & $\ldots$ & \\
\hline Net domestic credit & -13.3 & 18.4 & 39.0 & 5.9 & -1.1 & $\ldots$ & w. & $\ldots$ & w. & w. & \\
\hline Net credit to the government & -14.2 & 10.9 & 32.1 & 0.0 & -13.8 & $\ldots$ & $\ldots$ & $\ldots$ & $\ldots$ & $\ldots$ & \\
\hline Credit to the private sector & 0.6 & 8.0 & 7.5 & 5.7 & 11.3 & $\ldots$ & $\ldots$ & $\ldots$ & $\ldots$ & $\ldots$ & \\
\hline Credit to the parastatals & 0.4 & -0.4 & -0.6 & 0.2 & 1.4 & $\ldots$ & $\ldots$ & $\ldots$ & $\ldots$ & $\ldots$ & \\
\hline Central bank refinance rate (level in percent) & 24 & 15 & 8 & $\ldots$ & $\ldots$ & $\ldots$ & $\ldots$ & $\ldots$ & $\ldots$ & $\ldots$ & \\
\hline \multicolumn{12}{|c|}{ (In percent of GDP) } \\
\hline Central government finances & & & & & & & & & & & \\
\hline Revenue (excluding grants) & 7.9 & 8.2 & 7.7 & 9.0 & 9.2 & 10.1 & 10.3 & 11.0 & 11.0 & 11.5 & \\
\hline Grants (excluding humanitarian aid) & 0.4 & 1.9 & 2.0 & 4.3 & 5.9 & 6.8 & 10.0 & 8.8 & 8.2 & 8.0 & \\
\hline Expenditure 2/ & 10.3 & 13.2 & 13.6 & 17.6 & 19.3 & 20.2 & 25.3 & 21.5 & 21.6 & 21.4 & \\
\hline Domestic primary cash balance $3 /$ & 1.4 & 0.5 & -0.2 & 2.1 & 1.0 & 3.3 & 2.3 & 4.7 & 3.6 & 4.0 & \\
\hline Overall balance (commitment basis) & -2.0 & -3.1 & -3.9 & -4.3 & -4.2 & -3.3 & -5.0 & -1.7 & -2.4 & -1.8 & \\
\hline Overall consolidated cash balance 4/ & -0.1 & -1.4 & -2.0 & -3.0 & -3.2 & -1.5 & -3.2 & 0.3 & -0.5 & -0.2 & \\
\hline \multicolumn{12}{|l|}{ Investment and saving } \\
\hline Gross national savings & 11.2 & 11.1 & 13.4 & 13.3 & 16.6 & 15.8 & 21.1 & 18.9 & 20.3 & 20.4 & 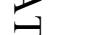 \\
\hline Government & 1.3 & -0.2 & -0.9 & 2.7 & 4.1 & 6.3 & 8.7 & 9.8 & 8.4 & 8.5 & $\rightrightarrows$ \\
\hline Nongovernment & 9.9 & 11.2 & 14.3 & 10.6 & 12.5 & 9.5 & 12.3 & 9.1 & 11.9 & 11.9 & $D D$ \\
\hline Gross domestic savings & 4.0 & 5.2 & 5.7 & 5.9 & 6.5 & 7.4 & 7.4 & 9.0 & 9.0 & 9.1 & $\Omega 0$ \\
\hline Government & 1.1 & 1.0 & 0.1 & 1.5 & 1.0 & 2.7 & 1.8 & 4.1 & 3.0 & 3.0 & \\
\hline Nongovernment & 3.0 & 4.2 & 5.6 & 4.5 & 5.5 & 4.7 & 5.7 & 4.9 & 5.9 & 6.1 & 30 \\
\hline Investment & 9.0 & 12.1 & 12.2 & 17.4 & 18.6 & 20.6 & 24.7 & 22.5 & 21.8 & 21.5 & $\vec{\pi} \leqslant$ \\
\hline Government 5/ & 1.0 & 2.6 & 2.7 & 6.9 & 8.1 & 9.6 & 13.7 & 11.5 & 10.8 & 10.3 & $Z=$ \\
\hline Nongovernment 6/ & 8.0 & 9.5 & 9.5 & 10.5 & 10.5 & 11.0 & 11.0 & 11.0 & 11.0 & 11.2 & $\exists x$ \\
\hline
\end{tabular}




\begin{tabular}{|c|c|c|c|c|c|c|c|c|c|c|}
\hline & \multirow[t]{2}{*}{2002} & \multicolumn{2}{|c|}{2003} & \multicolumn{2}{|c|}{2004} & \multicolumn{2}{|c|}{2005} & \multicolumn{2}{|c|}{2006} & \multirow{2}{*}{$\begin{array}{l}2007 \\
\text { Proj. }\end{array}$} \\
\hline & & $\begin{array}{c}\text { Country } \\
\text { Report No. } \\
04 / 97\end{array}$ & Est. & $\begin{array}{c}\text { Country } \\
\text { Report No. } \\
04 / 97\end{array}$ & Rev. Prog. & $\begin{array}{c}\text { Country } \\
\text { Report No. } \\
04 / 97\end{array}$ & Proj. & $\begin{array}{c}\text { Country } \\
\text { Report No. } \\
04 / 97\end{array}$ & Proj. & \\
\hline & \multicolumn{10}{|c|}{ (In millions of U.S. dollars; unless otherwise indicated) } \\
\hline \multicolumn{11}{|l|}{ Balance of payments } \\
\hline Exports of goods and nonfactor services & $1,174.2$ & $1,383.9$ & $1,428.9$ & $1,529.9$ & $1,588.7$ & $1,697.9$ & $1,680.9$ & $1,880.5$ & $1,815.9$ & $1,979.0$ \\
\hline (in percent of GDP) & 21.2 & 24.8 & 25.2 & 24.9 & 23.5 & 25.5 & 22.9 & 26.0 & 22.7 & 22.7 \\
\hline Imports of goods and nonfactor services & $1,446.5$ & $1,771.8$ & $1,801.4$ & $2,231.6$ & $2,410.0$ & $2,574.2$ & $2,946.3$ & $2,858.4$ & $2,839.8$ & 3,059.9 \\
\hline (in percent of GDP) & 26.1 & 31.7 & 31.7 & 36.3 & 35.7 & 38.6 & 40.2 & 39.5 & 35.5 & 35.1 \\
\hline $\begin{array}{l}\text { External current account, including grants, } \\
\text { before debt relief (in percent of GDP) }\end{array}$ & -2.8 & -2.0 & 0.6 & -6.0 & -3.3 & -7.6 & -6.5 & -7.6 & -5.4 & -5.3 \\
\hline $\begin{array}{l}\text { External current account, excluding grants, } \\
\text { before debt relief (in percent of GDP) }\end{array}$ & -10.3 & -10.7 & -8.1 & -14.7 & -13.3 & -16.8 & -18.7 & -16.7 & -13.7 & -12.0 \\
\hline $\begin{array}{l}\text { External current account, including grants, } \\
\text { after debt relief (in percent of GDP) 7/ }\end{array}$ & 2.2 & -1.1 & 1.2 & -4.1 & -2.0 & -4.7 & -3.6 & -3.5 & -1.5 & -1.2 \\
\hline Gross official reserves (end-of-period) & 74.7 & 156.6 & 96.6 & 272.1 & 209.3 & 382.2 & 318.2 & 479.1 & 479.1 & 611.9 \\
\hline $\begin{array}{l}\text { Gross official reserves (in weeks of non-aid-related } \\
\text { imports of goods and nonfactor services) }\end{array}$ & 2.9 & 5.3 & 3.3 & 8.2 & 6.2 & 10.7 & 8.9 & 12.1 & 11.4 & 14.1 \\
\hline \multicolumn{11}{|l|}{ External public debt } \\
\hline Total stock, including IMF 8/ & 10,659 & 10,627 & 10,563 & 10,714 & 10,783 & 10,970 & 11,032 & 5,687 & 5,512 & 5,744 \\
\hline Net present value of debt (NPV) $9 /$ & 7,933 & 7,376 & 7,274 & 7,538 & 7,546 & 7,837 & 7,756 & 2,095 & 1,859 & 2,009 \\
\hline $\begin{array}{l}\text { Scheduled debt service } 7 / \\
\text { In percent of exports of goods and }\end{array}$ & 34.2 & 139.9 & 126.7 & 140.3 & 91.7 & 204.9 & 135.0 & 313.5 & 198.7 & 226.7 \\
\hline nonfactor services & 2.9 & 10.1 & 8.9 & 9.2 & 5.8 & 12.1 & 8.0 & 16.7 & 10.9 & 11.5 \\
\hline In percent of government revenue, excluding grants & 7.4 & 24.8 & 23.0 & 17.3 & 9.0 & 18.2 & 9.1 & 21.9 & 12.9 & 13.3 \\
\hline \multicolumn{11}{|l|}{ Memorandum items: } \\
\hline Exchange rate & & & & & & & & & & \\
\hline $\begin{array}{l}\text { Units of local currency per U.S. dollar } \\
\text { (end-of-period) }\end{array}$ & 382.0 & 372.5 & 372.5 & & & & & & & \\
\hline Nominal GDP (in billions of Congo francs) & 1,922 & 2,270 & 2,299 & 2,537 & 2,565 & 2,851 & 2,881 & 3,189 & 3,237 & 3,637 \\
\hline (Percentage changes) & 36.6 & 18.5 & 19.6 & 11.7 & 11.6 & 12.4 & 12.4 & 11.9 & 12.4 & 12.4 \\
\hline
\end{tabular}

Sources: Congolese authorities; and IMF staff estimates Sources: Données fournies par les autorités congolaises; et estimations et projections des services du FMI.

$1 /$ Change in annual average. Minus sign indicates depreciation.

2/ Includes interest due on external debt (including debt service on rescheduling) and, from 2003 onward, expenditure financed by resources released under the enhanced HIPC Initiative.

3/ Revenue (excluding grants) minus expenditure (excluding interest on debt, foreign-financed expenditure, and HIPC-related expenditure). 4/ Cash balance after interest rescheduling (including HIPC).

5/ From 2003 onward, includes investment financed by resources released under the enhanced HIPC Initiative.

6/ From 2003 onward, includes capital projects financed by NGOs.

7/ From 2003, after debt relief from bilateral creditors and HIPC Initiative assistance.

8/ End-of-period debt stock, including arrears and after HIPC Initiative assistance.

9/ Estimates and projections based on end-2002 DSA and after HIPC Initiative assistance. 


\begin{tabular}{|c|c|c|c|c|c|c|c|c|}
\hline & \multicolumn{2}{|c|}{ January-September } & \multicolumn{2}{|c|}{ October-December } & \multicolumn{4}{|c|}{2003} \\
\hline & $\begin{array}{c}\text { Country } \\
\text { Report No. } \\
04 / 97\end{array}$ & In $\%$ of GDP & $\begin{array}{c}\text { Country } \\
\text { Report No. } \\
04 / 97\end{array}$ & Est. & $\begin{array}{c}\text { Country } \\
\text { Report No. } \\
04 / 97\end{array}$ & In $\%$ of GDP & Est. & In $\%$ of GDP \\
\hline Total revenue and grants & 150,566 & 6.6 & 78,681 & 73,133 & 229,247 & 10.1 & 223,699 & 9.7 \\
\hline Total revenue & 127,095 & 5.5 & 58,761 & 50,098 & 185,856 & 8.2 & 177,193 & 7.7 \\
\hline Customs and excise (OFIDA) & 50,426 & 2.2 & 26,360 & 22,074 & 76,786 & 3.4 & 72,500 & 3.2 \\
\hline DGRAD (including revenue from public enterprises) & 12,387 & 0.5 & 3,106 & 2,706 & 15,493 & 0.7 & 15,093 & 0.7 \\
\hline GECAMINES & 0 & 0.0 & 0 & 0 & 0 & 0.0 & 0 & 0.0 \\
\hline MIBA & 1,004 & 0.0 & 2,000 & 0 & 3,004 & 0.1 & 1,004 & 0.0 \\
\hline Petroleum & 26,238 & 1.1 & 10,470 & 10,147 & 36,708 & 1.6 & 36,385 & 1.6 \\
\hline Production & 21,698 & 0.9 & 10,464 & 10,141 & 32,162 & 1.4 & 31,839 & 1.4 \\
\hline Distribution & 4,541 & 0.2 & 6 & 6 & 4,547 & 0.2 & 4,547 & 0.2 \\
\hline Other & 596 & 0.0 & 100 & 477 & 696 & 0.0 & 1,073 & 0.0 \\
\hline Total grants & 23,471 & 1.0 & 19,920 & 23,035 & 43,391 & 1.9 & 46,506 & 2.0 \\
\hline Of which : project grants & 23,471 & 1.0 & 6,278 & 16,530 & 29,749 & 1.3 & 40,001 & 1.7 \\
\hline Total expenditure 1/ & 189,017 & 8.2 & 72,851 & 80,770 & 261,868 & 11.5 & 269,787 & 11.7 \\
\hline Current expenditure & 125,741 & 5.5 & 52,540 & 53,847 & 178,281 & 7.9 & 179,588 & 7.8 \\
\hline Wages & 36,547 & 1.6 & 14,964 & 20,558 & 51,511 & 2.3 & 57,105 & 2.5 \\
\hline Military and police & 14,767 & 0.6 & 6,997 & 8,284 & 21,764 & 1.0 & 23,051 & 1.0 \\
\hline Civilians & 21,779 & 0.9 & 7,967 & 12,274 & 29,746 & 1.3 & 34,053 & 1.5 \\
\hline Interest payments & 12,463 & 0.5 & 17,410 & 9,875 & 29,873 & 1.3 & 22,338 & 1.0 \\
\hline External debt (interest payment after debt relief) 2/ & 11,503 & 0.5 & 17,218 & 7,868 & 28,721 & 1.3 & 19,371 & 0.8 \\
\hline Domestic debt (interest payment) & 960 & 0.0 & 192 & 2,007 & 1,152 & 0.1 & 2,967 & 0.1 \\
\hline Other current expenditure & 62,512 & 2.7 & 14,379 & 17,766 & 76,891 & 3.4 & 80,278 & 3.5 \\
\hline Institutions & 21,574 & 0.9 & 4,805 & 6,674 & 26,379 & 1.2 & 28,248 & 1.2 \\
\hline Ministries & 26,375 & 1.1 & 5,566 & 7,183 & 31,941 & 1.4 & 33,558 & 1.5 \\
\hline Centralized payments (utilities) & 9,542 & 0.4 & 2,552 & 2,022 & 12,094 & 0.5 & 11,564 & 0.5 \\
\hline Provinces & 5,020 & 0.2 & 1,456 & 1,887 & 6,476 & 0.3 & 6,907 & 0.3 \\
\hline Other & 0 & 0.0 & 0 & 0 & 0 & 0.0 & 0 & 0.0 \\
\hline Transfers and subsidies & 14,219 & 0.6 & 5,787 & 5,648 & 20,006 & 0.9 & 19,867 & 0.9 \\
\hline MIBA and GECAMINES & 8,412 & 0.4 & 3,148 & 3,148 & 11,560 & 0.5 & 11,560 & 0.5 \\
\hline Transfers to public agencies and budgets annexes & 491 & 0.0 & 157 & 123 & 648 & 0.0 & 614 & 0.0 \\
\hline Retrocessions to revenue-collecting agencies & 5,316 & 0.2 & 2,482 & 2,377 & 7,798 & 0.3 & 7,693 & 0.3 \\
\hline Foreign-financed investment & 25,058 & 1.1 & 18,273 & 22,096 & 43,331 & 1.9 & 47,154 & 2.1 \\
\hline Congolese-financed investment & 11,080 & 0.5 & 4,098 & 3,523 & 15,177 & 0.7 & 14,603 & 0.6 \\
\hline Net lending & 0 & 0.0 & 0 & 0 & 0 & 0.0 & 0 & 0.0 \\
\hline Other operations & 89 & 0.0 & 0 & 0 & 89 & 0.0 & 89 & 0.0 \\
\hline HIPC-related expenditure & 0 & 0.0 & 0 & 0 & 0 & 0.0 & 0 & 0.0 \\
\hline Repayment of arrears & 7,167 & 0.3 & 724 & 657 & 7,891 & 0.3 & 7,824 & 0.3 \\
\hline $\mathrm{BCC}$ treasury deficit & 17,783 & 0.8 & $-2,783$ & 647 & 15,000 & 0.7 & 18,430 & 0.8 \\
\hline Unconsolidated domestic primary balance (cash basis) & $-6,618$ & -0.3 & 18,810 & 1,946 & 12,191 & 0.5 & $-4,672$ & -0.2 \\
\hline Consolidated primary balance (cash basis) & $-25,988$ & -1.1 & 23,240 & 2,238 & $-2,749$ & -0.1 & $-23,750$ & -1.0 \\
\hline Unconsolidated balance (cash basis) & $-20,668$ & -0.9 & 3,047 & $-6,990$ & $-17,621$ & -0.8 & $-27,658$ & -1.2 \\
\hline Consolidated balance (cash basis) & $-38,451$ & -1.7 & 5,830 & $-7,637$ & $-32,621$ & -1.4 & $-46,088$ & -2.0 \\
\hline Total financing & 38,451 & 1.7 & $-5,830$ & 7,637 & 32,621 & 1.4 & 46,088 & 2.0 \\
\hline Nonbank (certificates of deposit net) & -344 & 0.0 & 0 & 0 & -344 & 0.0 & -344 & 0.0 \\
\hline $\mathrm{BCC}$ and other banking system $3 /$ & 22,570 & 1.0 & $-13,026$ & 5,525 & 9,544 & 0.4 & 28,095 & 1.2 \\
\hline Foreign financing & 15,827 & 0.7 & 6,484 & 2,315 & 22,311 & 1.0 & 18,142 & 0.8 \\
\hline Nondomestic nonresident bank & 0 & 0.0 & 0 & 0 & 0 & 0.0 & 0 & 0.0 \\
\hline Amortization (net payment) 2/ & $-17,865$ & -0.8 & $-21,511$ & $-19,779$ & $-39,376$ & -1.7 & $-37,644$ & -1.6 \\
\hline Additional financing & 33,692 & 1.5 & 27,995 & 22,094 & 61,687 & 2.7 & 55,786 & 2.4 \\
\hline Of which: project loans & 3,880 & 0.2 & 11,995 & 5,574 & 15,875 & 0.7 & 9,454 & 0.4 \\
\hline Of which: budget loans & 29,812 & 1.3 & 16,000 & 16,520 & 45,812 & 2.0 & 46,332 & 2.0 \\
\hline Discrepancy & 398 & 0.0 & 712 & -203 & 1,110 & 0.0 & 195 & 0.0 \\
\hline
\end{tabular}

Sources: Congolese authortities; and IMF staff estimates and projections.

1/ Including domestic arrears and BCC operations. A surplus of the BCC appears as a minus.

2/ Includes deposits of HIPC resources at the BCC in October and December.

3/ Net banking system credit to the government plus treasury balance of the central bank. This amount has been audited and includes about CGF 1.5 billion (0.1 percent of GDP, which has not been taken into account in the monetary survey. 
Table 2B. Democratic Republic of the Congo: Monthly Treasury Cash-Flow Plan, 2004

\begin{tabular}{|c|c|c|c|c|c|c|c|c|c|c|}
\hline & \multicolumn{4}{|c|}{ January-March } & \multicolumn{2}{|c|}{ April } & \multicolumn{2}{|c|}{ May } & \multicolumn{2}{|c|}{ June } \\
\hline & $\begin{array}{c}\text { Country } \\
\text { Report No. } \\
04 / 97\end{array}$ & $\begin{array}{c}\text { In \% } \\
\text { of GDP }\end{array}$ & Est. & $\begin{array}{r}\text { In \% } \\
\text { of GDP }\end{array}$ & $\begin{array}{c}\text { Country } \\
\text { Report No. } \\
04 / 97\end{array}$ & $\begin{array}{l}\text { Rev. } \\
\text { Prog. }\end{array}$ & $\begin{array}{c}\text { Country } \\
\text { Report No. } \\
04 / 97\end{array}$ & $\begin{array}{l}\text { Rev. } \\
\text { Prog. }\end{array}$ & $\begin{array}{c}\text { Country } \\
\text { Report No. } \\
04 / 97\end{array}$ & $\begin{array}{l}\text { Rev. } \\
\text { prog. }\end{array}$ \\
\hline Total revenue and grants & 76,116 & 3.0 & 92,036 & 3.6 & 25,496 & 27,408 & 25,155 & 26,682 & 24,931 & 27,428 \\
\hline Total revenue & 51,723 & 2.0 & 57,886 & 2.3 & 17,758 & 18,073 & 17,104 & 17,347 & 18,007 & 18,246 \\
\hline Customs and excise (OFIDA) & 21,720 & 0.9 & 23,710 & 0.9 & 8,362 & 8,142 & 8,688 & 8,467 & 9,448 & 9,227 \\
\hline Direct and indirect taxes (DGC) & 15,141 & 0.6 & 18,332 & 0.7 & 4,256 & $\begin{array}{l}0,142 \\
3,902\end{array}$ & 4,247 & 3,893 & 4,325 & 3,971 \\
\hline DGRAD (including revenue from public enterprises) & 6,145 & 0.2 & 4,848 & 0.2 & 2,217 & 2,548 & 1,237 & 1,505 & 1,294 & 1,565 \\
\hline $\begin{array}{l}\text { GECAMINES } \\
\end{array}$ & 0 & 0.0 & 0 & 0.0 & 0 & 0 & 0 & 0 & 0 & 0 \\
\hline MIBA & 1,250 & 0.0 & 0 & 0.0 & 417 & 556 & 417 & 556 & 417 & 556 \\
\hline Petroleum & 7,467 & 0.3 & 10,569 & 0.4 & 2,506 & 2,926 & 2,514 & 2,926 & 2,522 & 2,926 \\
\hline Production & 7,467 & 0.3 & 10,569 & 0.4 & 2,506 & 2,926 & 2,514 & 2,926 & 2,522 & 2,926 \\
\hline Distribution & 0 & 0.0 & 0 & 0.7 & 0 & 0 & 0 & $\begin{aligned} 2,20 \\
0\end{aligned}$ & 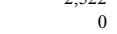 & 2,20 \\
\hline Other & 0 & 0.0 & 427 & 0.0 & 0 & 0 & 0 & 0 & 0 & 0 \\
\hline Off-budget revenue & 0 & 0.0 & 0 & 0.0 & & & & & & \\
\hline Total grants & 24,393 & 1.0 & 34,150 & 1.3 & 7,738 & 9,335 & 8,051 & 9,335 & 6,924 & 9,183 \\
\hline Of which : project grants & 12,446 & 0.5 & 11,452 & 0.4 & 4,258 & 7,338 & 4,272 & 7,338 & 4,369 & 7,186 \\
\hline Of which: HIPC debt relief & 11,946 & 0.5 & 22,698 & 0.9 & 3,480 & 1,996 & 3,779 & 1,996 & 2,555 & 1,996 \\
\hline Total expenditure 1/ & 92,789 & 3.7 & 67,473 & 2.6 & 32,600 & 38,758 & 33,839 & 36,259 & 34,526 & 35,164 \\
\hline Current expenditure & 51,342 & 2.0 & 45,769 & 1.8 & 16,750 & 20,456 & 17,502 & 17,651 & 17,059 & 16,900 \\
\hline Wages & 17,961 & 0.7 & 14,110 & 0.6 & 6,654 & 9,839 & 6,654 & 8,069 & 6,654 & 8,069 \\
\hline Military and police & 7,773 & 0.3 & 6,086 & 0.2 & 2,591 & 4,349 & 2,591 & 2,662 & 2,591 & 2,662 \\
\hline Civilians & 10,188 & 0.4 & 8,024 & 0.3 & 4,063 & 5,490 & 4,063 & 5,407 & 4,063 & 5,407 \\
\hline Interest payments & $\begin{array}{r}9,055 \\
9050\end{array}$ & 0.4 & $\begin{array}{l}8,024 \\
8,657\end{array}$ & 0.3 & 1,974 & 2,806 & 2,793 & 1,964 & 2,232 & 1,302 \\
\hline External debt (interest payment after debt relief) $2 /$ & 8,555 & 0.3 & 7,925 & 0.3 & 1,807 & 2,665 & 2,626 & 1,823 & 2,065 & 1,161 \\
\hline Domestic debt (interest payment) & 500 & 0.0 & 732 & 0.0 & 167 & 141 & 167 & 141 & 167 & 141 \\
\hline Other current expenditure & 21,598 & 0.9 & 20,620 & 0.8 & 7,078 & 6,613 & 7,078 & 6,613 & 7,278 & 6,613 \\
\hline Institutions & 4,500 & 0.2 & 5,637 & 0.2 & 1,500 & 1,281 & 1,500 & 1,281 & 1,500 & 1,281 \\
\hline Ministries & 7,900 & 0.3 & 9,578 & 0.4 & 2,500 & 2,382 & 2,500 & 2,382 & 2,700 & 2,382 \\
\hline Centralized payments (utilities) & 6,342 & 0.2 & 3,226 & 0.1 & 2,123 & 1,834 & 2,123 & 1,834 & 2,123 & 1,834 \\
\hline Provinces & 2,856 & 0.1 & 2,179 & 0.1 & 956 & 1,116 & 956 & 1,116 & 956 & 1,116 \\
\hline Other & 0 & 0.0 & 0 & 0.0 & 0 & 0 & 0 & 0 & 0 & 0 \\
\hline Transfers and subsidies & 2,728 & 0.1 & 2,382 & 0.1 & 1,044 & 1,199 & 978 & 1,005 & 896 & 917 \\
\hline $\begin{array}{l}\text { MIBA and GECAMINES } \\
\text { GECA }\end{array}$ & 0 & 0.0 & 0 & 0.0 & 0 & 0 & 0 & 0 & 0 & 0 \\
\hline Transfers to public agencies and budgets annexes & 375 & 0.0 & 167 & 0.0 & 125 & 148 & 125 & 148 & 125 & 148 \\
\hline Retrocessions to revenue collecting agencies & 2,353 & 0.1 & 2,215 & 0.1 & 919 & 1,051 & 853 & 857 & 771 & 769 \\
\hline Capital expenditure & 31,510 & 1.2 & 18,757 & 0.7 & 10,990 & 11,705 & 11,230 & 12,269 & 12,790 & 12,354 \\
\hline Foreign-financed investment & 29,585 & 1.2 & 16,802 & 0.7 & 10,348 & 11,066 & 10,588 & 11,484 & 12,148 & 11,712 \\
\hline Congolese-financed investment & 1,925 & 0.1 & 1,955 & 0.1 & 642 & 638 & 642 & 784 & 642 & 642 \\
\hline HIPC-related expenditure & 5,541 & 0.2 & 0 & 0.0 & 3,694 & 3,694 & 3,694 & 4,926 & 3,694 & 4,926 \\
\hline Repayment of arrears $3 /$ & 0 & 0.0 & 289 & 0.0 & 0 & 0 & 0 & 0 & 0 & 0 \\
\hline $\mathrm{BCC}$ treasury deficit & 4,396 & 0.2 & 2,658 & 0.1 & 1,166 & 2,903 & 1,413 & 1,413 & 983 & 984 \\
\hline Unconsolidated domestic primary balance (cash basis) & 7,512 & 0.3 & 18,530 & 0.7 & 2,340 & -216 & 1,752 & 876 & 2,537 & 2,005 \\
\hline Consolidated primary balance (cash basis) & $-7,617$ & -0.3 & 33,220 & 1.3 & $-5,130$ & $-8,545$ & $-5,891$ & $-7,613$ & $-7,364$ & $-6,435$ \\
\hline Unconsolidated balance (cash basis) & $-12,276$ & -0.5 & 27,221 & 1.1 & $\begin{array}{l}-5,938 \\
-5,938\end{array}$ & $-8,447$ & 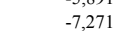 & $-8,164$ & $-8,612$ & $-6,752$ \\
\hline Consolidated balance (cash basis) & $-16,672$ & -0.7 & 24,563 & 1.0 & $-7,104$ & $-11,350$ & $-8,684$ & $-9,577$ & $-9,595$ & $-7,736$ \\
\hline Total financing & 16,672 & 0.7 & $-24,563$ & -1.0 & 7,104 & 11,350 & 8,684 & 9,577 & 9,595 & 7,736 \\
\hline Nonbank (certificates of deposit net) & 0 & 0.0 & 0 & 0.0 & & & & & & \\
\hline BCC and other banking system $4 /$ & $-3,941$ & -0.2 & $-9,346$ & -0.4 & 4,742 & 12,100 & 6,196 & 7,645 & $-4,922$ & $-27,246$ \\
\hline $\begin{array}{l}\text { Foreign financing } \\
\text { F }\end{array}$ & 20,614 & 0.8 & $-16,546$ & -0.6 & 2,362 & -750 & 2,489 & 1,932 & 14,518 & 34982 \\
\hline Nondomestic nonresident bank & & & & & & & & & & \\
\hline Amortization (net payment) $2 /$ & $-23,538$ & -1 & $-24,223$ & -0.9 & $-3,728$ & $-4,478$ & $-3,827$ & $-2,215$ & $-3,565$ & $-1,844$ \\
\hline Additional financing & 44,152 & 2 & 7,677 & 0.3 & 6,090 & 3,728 & 6,316 & 4,146 & 18,083 & 36,826 \\
\hline Of which: project loans & 17,138 & 1 & 6,666 & 0.3 & 6,090 & 3,728 & 6,316 & 4,146 & 7,779 & 4,526 \\
\hline Of which: budget loans & 27,014 & 1 & 1,011 & 0.0 & 0 & 0 & 0 & 0 & 10,304 & 32,300 \\
\hline Discrepancy & 0 & 0 & 1,328 & 0.1 & 0 & 0 & 0 & 0 & 0 & 0 \\
\hline
\end{tabular}


Table 2B. Democratic Republic of the Congo: Monthly Treasury Cash-Flow Plan, 2004

(In millions of Congo francs)

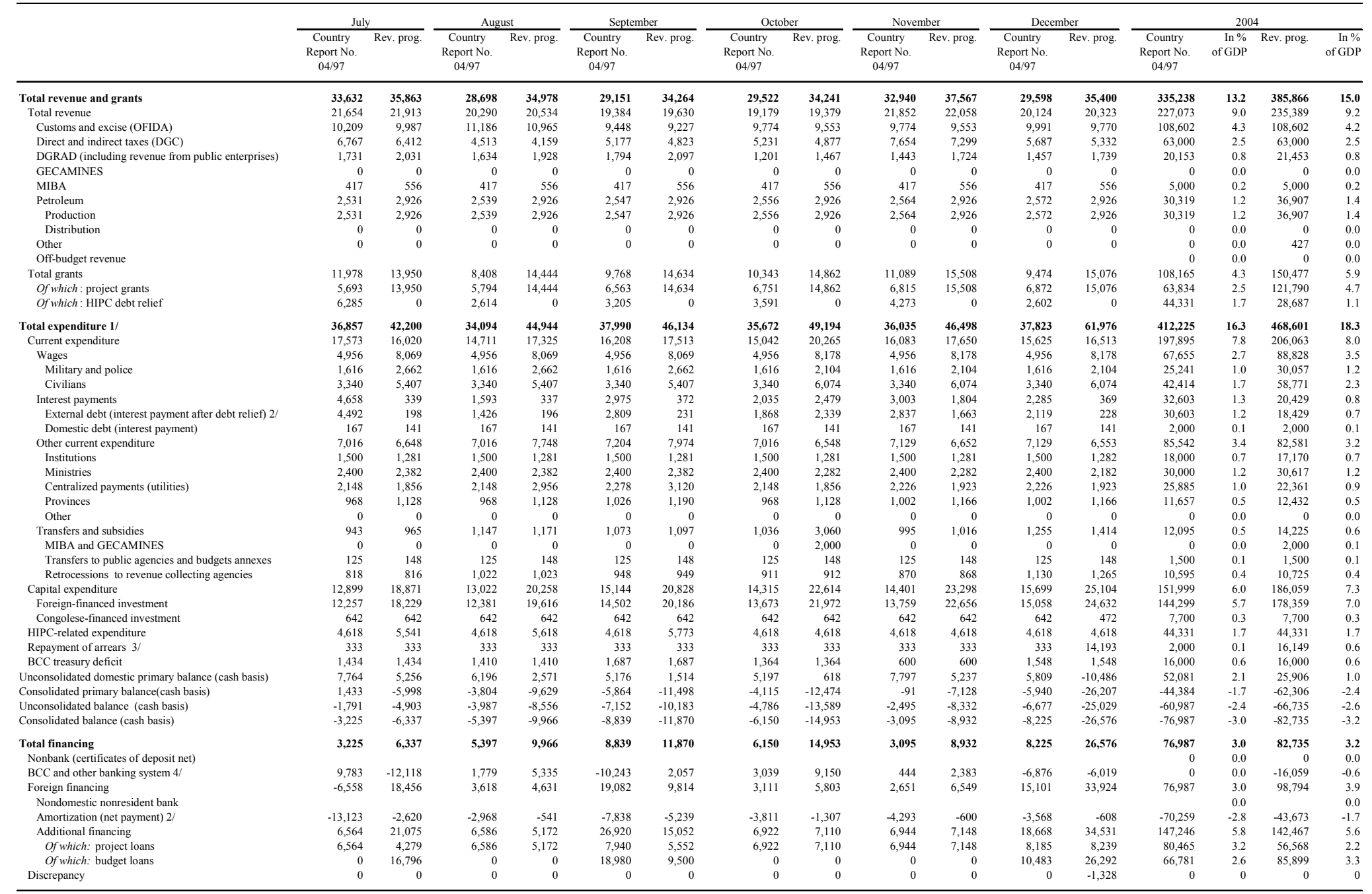

Sources: Congolese authortities; and IMF staff estimates and projections.

1/ Including the repayment of domestic arrears and the balance of the BCC (for the latter, a minus sign corresponds to an excedent).

2) Including deposits to the HIPC special account at the BCC 
Table 3. Action Plan of the Central Bank of the Congo

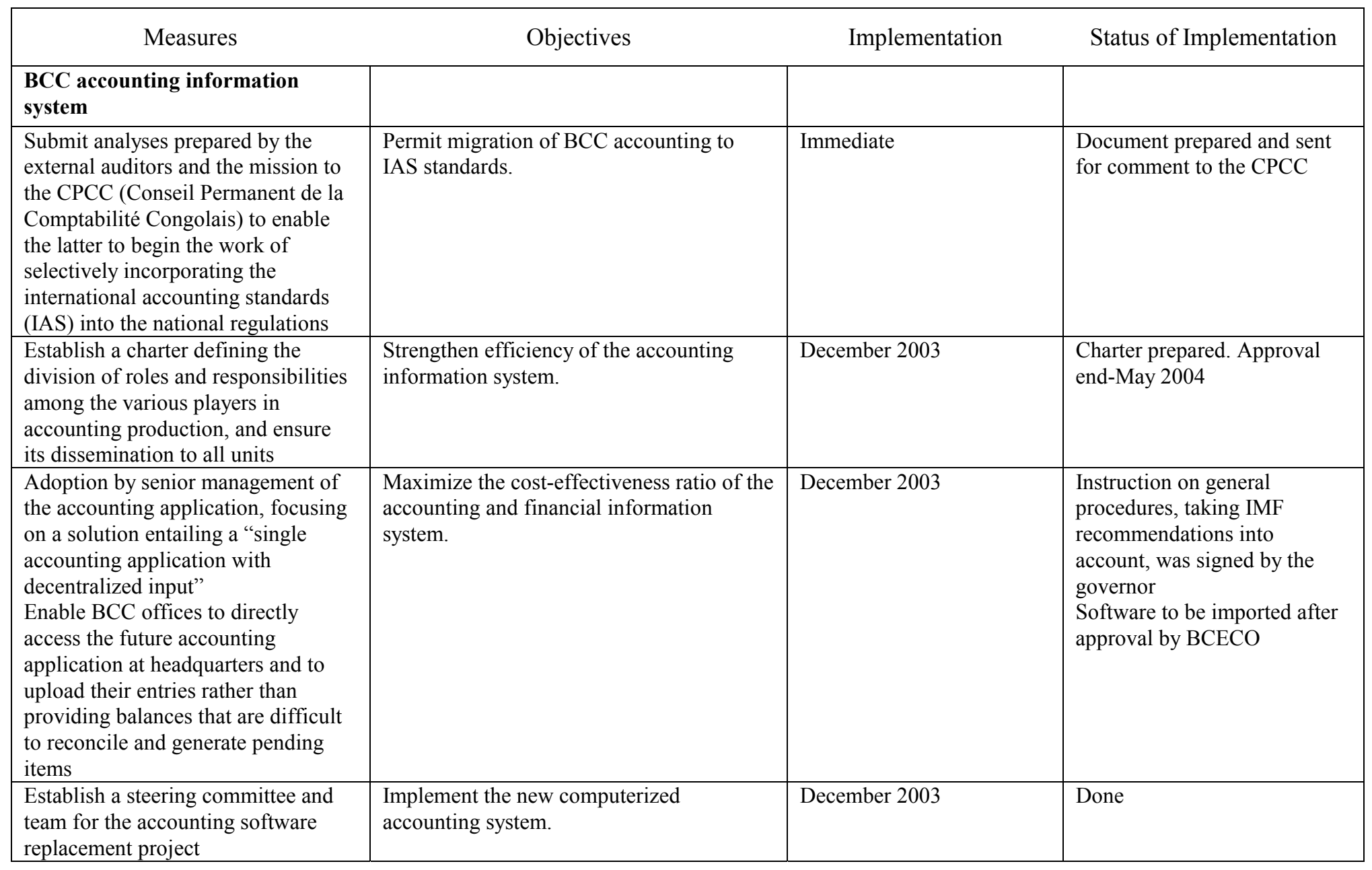


Table 3. Action Plan of the Central Bank of the Congo

\begin{tabular}{|c|c|c|c|}
\hline Measures & Objectives & Implementation & Status of Implementation \\
\hline $\begin{array}{l}\text { Reorganization of the departments } \\
\text { responsible for government accounts } \\
\text { within the BCC }\end{array}$ & $\begin{array}{l}\text { Ensure the unity of government cashier } \\
\text { operations. }\end{array}$ & June 2004 & In progress \\
\hline \multicolumn{4}{|l|}{$\begin{array}{l}\text { Strengthening of financial } \\
\text { intermediation }\end{array}$} \\
\hline $\begin{array}{l}\text { Irrevocably commit to guaranteeing } \\
\text { the convertibility of banks' free } \\
\text { reserves into currency and launch a } \\
\text { communications campaign in this } \\
\text { connection }\end{array}$ & $\begin{array}{l}\text { Normal operation of banks' accounts with } \\
\text { the BCC is an initial condition for } \\
\text { development of financial intermediation. }\end{array}$ & Ongoing & Done \\
\hline $\begin{array}{l}\text { Establish a strategy for } \\
\text { communication and dialogue with } \\
\text { private transactors }\end{array}$ & $\begin{array}{l}\text { Encourage private initiative, reduce the } \\
\text { uncertainty that private operators face, and } \\
\text { increase the quality and acceptance of the } \\
\text { authorities' decisions. }\end{array}$ & Ongoing & Done \\
\hline \multicolumn{4}{|l|}{ Financial system } \\
\hline $\begin{array}{l}\text { Explicitly assign bank supervision } \\
\text { staff to one of the two functions (off- } \\
\text { site supervision or on-site } \\
\text { supervision) }\end{array}$ & $\begin{array}{l}\text { Improve professionalism and the } \\
\text { acquisition of specialized experience. }\end{array}$ & December 2003 & Done \\
\hline $\begin{array}{l}\text { Continue the work of preparing the } \\
\text { new system for generating periodic } \\
\text { statements reflecting the reform of } \\
\text { the chart of accounts for banks by } \\
\text { the CPCC as part of the project to } \\
\text { implement the computerized Bank } \\
\text { Supervision Application (BSA) }\end{array}$ & $\begin{array}{l}\text { Provide the supervision authorities with } \\
\text { reliable, relevant, and timely information. }\end{array}$ & September 2004 & In progress \\
\hline $\begin{array}{l}\text { Strengthen on-site supervision by } \\
\text { providing remote assistance to an on- } \\
\text { site supervision mission }\end{array}$ & $\begin{array}{l}\text { Enhance the level of competence of the } \\
\text { inspectors. }\end{array}$ & During 2004 & In progress \\
\hline
\end{tabular}


Table 3. Action Plan of the Central Bank of the Congo

\begin{tabular}{|c|c|c|c|}
\hline Measures & Objectives & Implementation & Status of Implementation \\
\hline $\begin{array}{l}\text { Formalize the operating mechanism } \\
\text { for off-site supervision and } \\
\text { implement a normalized framework } \\
\text { for permanent files }\end{array}$ & $\begin{array}{l}\text { Ensure continuous monitoring of banks and } \\
\text { facilitate the detection of irregularities so } \\
\text { that corrective measures can be taken. }\end{array}$ & June 2004 & In progress \\
\hline $\begin{array}{l}\text { Implement a formalized procedure } \\
\text { for addressing irregularities detected } \\
\text { in the situation of credit institutions }\end{array}$ & $\begin{array}{l}\text { Ensure that penalties are applied } \\
\text { consistently and that the application of } \\
\text { corrective measures is monitored. }\end{array}$ & March 2004 & Not done \\
\hline $\begin{array}{l}\text { Establish a BSA steering committee } \\
\text { and project team }\end{array}$ & $\begin{array}{l}\text { Ensure that the timetable for BSA } \\
\text { deployment is followed. }\end{array}$ & December 2003 & Done \\
\hline $\begin{array}{l}\text { Establish an RMA (multi-application } \\
\text { network) steering committee and } \\
\text { project team }\end{array}$ & $\begin{array}{l}\text { Manage the increase in remote } \\
\text { transmissions of periodic statements. }\end{array}$ & December 2003 & Done \\
\hline $\begin{array}{l}\text { Establish a joint working group with } \\
\text { the BCC, CPCC, banks, and } \\
\text { Congolese accounting firms to } \\
\text { prepare the new chart of accounts } \\
\text { applicable to banks }\end{array}$ & $\begin{array}{l}\text { Provide the banking system with an } \\
\text { updated chart of accounts that guarantees } \\
\text { the reliability of financial information given } \\
\text { to third parties and sent to the DSIF. }\end{array}$ & December 2003 & Done \\
\hline \multicolumn{4}{|l|}{$\begin{array}{l}\text { Monetary policy and liquidity } \\
\text { management }\end{array}$} \\
\hline $\begin{array}{l}\text { Complete a study on restructuring } \\
\text { the BCC balance sheet to assess the } \\
\text { remuneration of government } \\
\text { securities to cover any losses as } \\
\text { required to ensure sustainability of } \\
\text { the operating account }\end{array}$ & $\begin{array}{l}\text { Strengthen the BCC's operational } \\
\text { autonomy. }\end{array}$ & December 2003 & In progress \\
\hline $\begin{array}{l}\text { Purchase CGF banknotes and inputs } \\
\text { through requests for competitive bids } \\
\text { from several suppliers }\end{array}$ & Reduce printing costs. & Immediate & In progress \\
\hline $\begin{array}{l}\text { Preparation by the Research } \\
\text { Directorate of Table 7, "Monitoring } \\
\text { Liquidity in CGF" }\end{array}$ & $\begin{array}{l}\text { Facilitate implementation of liquidity } \\
\text { management. }\end{array}$ & Immediate & $\begin{array}{l}\text { In progress; most directorates } \\
\text { are already submitting the data }\end{array}$ \\
\hline
\end{tabular}


Table 3. Action Plan of the Central Bank of the Congo

\begin{tabular}{|c|c|c|c|}
\hline Measures & Objectives & Implementation & Status of Implementation \\
\hline $\begin{array}{l}\text { Abolish the ceiling on refinancing } \\
\text { allocated to each bank }\end{array}$ & The ratio is no longer relevant. & $\begin{array}{l}\text { Simultaneously with the } \\
\text { reform of guarantees }\end{array}$ & Done \\
\hline $\begin{array}{l}\text { Accept only foreign exchange as } \\
\text { collateral for BCC refinancing }\end{array}$ & $\begin{array}{l}\text { Improve the BCC's protection against } \\
\text { counterparty risk. }\end{array}$ & March 2004 & Nearing completion \\
\hline $\begin{array}{l}\text { Consolidate into a single 24-hour } \\
\text { window the "call money" and } \\
\text { "current account advances" } \\
\text { windows. Accept only foreign } \\
\text { exchange as collateral. In the } \\
\text { exceptional case when loans are not } \\
\text { guaranteed with foreign exchange } \\
\text { (debit balance at close of business), } \\
\text { charge a higher/deterrent rate }\end{array}$ & $\begin{array}{l}\text { Improve the quality of guarantees for } \\
\text { refinancing purposes. }\end{array}$ & March 2004 & Done \\
\hline $\begin{array}{l}\text { Replace the rediscount window with } \\
\text { a permanent foreign exchange swap } \\
\text { facility not to exceed seven days. } \\
\text { Foreign exchange would initially be } \\
\text { delivered in cash. }\end{array}$ & $\begin{array}{l}\text { Improve the BCC's protection against } \\
\text { counterparty risk. }\end{array}$ & December 2003 & $\begin{array}{l}\text { Not done, preparations in } \\
\text { progress }\end{array}$ \\
\hline \multicolumn{4}{|l|}{$\begin{array}{l}\text { Reserve management and } \\
\text { exchange operations }\end{array}$} \\
\hline $\begin{array}{l}\text { Fine-tune the methodology used to } \\
\text { compile statistics on the exchange } \\
\text { market }\end{array}$ & $\begin{array}{l}\text { Eliminate double counting and make a } \\
\text { distinction between customer and interbank } \\
\text { operations. }\end{array}$ & December 2003 & Done \\
\hline $\begin{array}{l}\text { Reduce the number of local banks } \\
\text { with which foreign exchange } \\
\text { accounts are opened from nine to } \\
\text { three }\end{array}$ & $\begin{array}{l}\text { Reduce the costs of bookkeeping by the } \\
\text { DSE and the related workload. }\end{array}$ & March 2004 & $\begin{array}{l}\text { Not done, because the } \\
\text { reduction will have a negative } \\
\text { impact on collection of duties } \\
\text { and taxes owed to the } \\
\text { government. }\end{array}$ \\
\hline $\begin{array}{l}\text { Sell foreign exchange through } \\
\text { single-rate competitive auctions }\end{array}$ & $\begin{array}{l}\text { Improve transparency and ensure equal } \\
\text { treatment of counterparties. }\end{array}$ & December 2003 & Done \\
\hline $\begin{array}{l}\text { Finalize the trading room procedures } \\
\text { manual }\end{array}$ & $\begin{array}{l}\text { Comply with international accounting } \\
\text { standards. }\end{array}$ & March 2004 & Done \\
\hline
\end{tabular}


Table 3. Action Plan of the Central Bank of the Congo

\begin{tabular}{|c|c|c|c|}
\hline Measures & Objectives & Implementation & Status of Implementation \\
\hline $\begin{array}{l}\text { Establish a steering committee and } \\
\text { project team for the trading room } \\
\text { application (front/back office) }\end{array}$ & $\begin{array}{l}\text { Effectively coordinate implementation of } \\
\text { the trading room application. }\end{array}$ & End-November 2003 & Done \\
\hline $\begin{array}{l}\text { Implement the IT infrastructure } \\
\text { (hardware) for the trading room }\end{array}$ & $\begin{array}{l}\text { Implementation will permit application of } \\
\text { the trading room procedures manual. }\end{array}$ & 1st half 2004 & $\begin{array}{l}\text { Hardware available now; } \\
\text { installation to be completed in } \\
\text { June } 2004\end{array}$ \\
\hline \multicolumn{4}{|l|}{$\begin{array}{l}\text { Information technology and } \\
\text { project management }\end{array}$} \\
\hline $\begin{array}{l}\text { Establish project teams and a project } \\
\text { steering committee to supervise them }\end{array}$ & $\begin{array}{l}\text { Improve project management and } \\
\text { systematize relations between users and } \\
\text { computer specialists. }\end{array}$ & $\begin{array}{l}\text { Beginning December 2003, } \\
\text { for all computer } \\
\text { applications }\end{array}$ & Done \\
\hline Establish an IT committee & Prepare and manage the IT strategy. & December 2003 & Done \\
\hline $\begin{array}{l}\text { Standardize computer hardware and } \\
\text { software to the extent possible }\end{array}$ & Facilitate IT management. & Ongoing & In progress \\
\hline $\begin{array}{l}\text { Provide adequate training for IT } \\
\text { specialists and users }\end{array}$ & Optimize the implementation and use of IT. & Ongoing & In progress \\
\hline
\end{tabular}




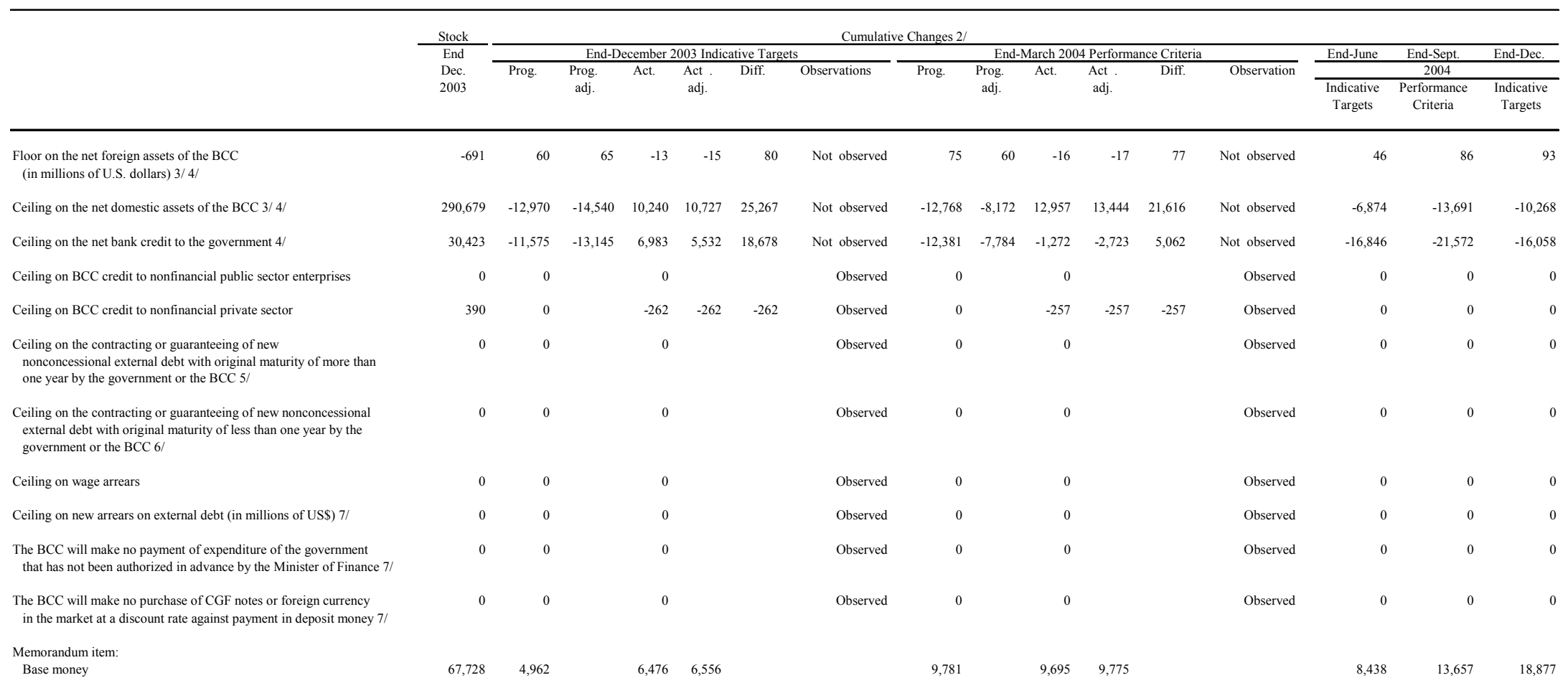

Source: Congolese authorities.

Note: Until the expiration of the three-year arrangement under the PRGF in June 2005 , the observance of the first five performance criteria will be audited by an international firm.

$1 /$ Quantitative performance criteria and benchmarks, as well as the procedures for their monitoring are defined in the attached Technical Memorandum of Understanding.

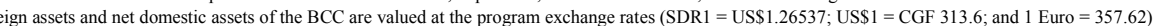

4 Fifty percent of any surplus over the programmed amount of external budgetary assistance (excluding project assistance) net of debt service and including external debt service rescheduling, that has not been used to finance poverty reduction expenditure, public enterprise restructuring, and domestic debt repayment (including cross-arrears on payments that have been certified in cooperation with World Bank staff) will be used to reduce net banking system credit to the government, and the corresponding performance criterion will be lowered accordingly The criteria on net foreign assets and net domestic assets will be raised and lowered, respectively, by the same amount. This adjustment does not apply to HIPC resources, which will be deposited in a special account at the BCC.

$5 /$ This performance criterion applies not only to debt as defined in item №. 9 of the Guidelines on Performance Criteria with Respect to Foreign Debt, adopted on August 24, 2000, but also to commitments contracted or guaranteed for which value has not been received. Excluded from this performance criterion are rescheduling arrangements and purchases from the Fund. For purposes of this performance criterion, the term "nonconcesssional" means that the debt has a grant element of less than 35 percent, calculated using currency-specific discount rates that are

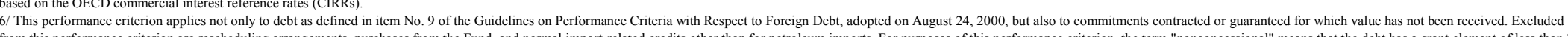
from this perfor

$7 /$ These are continuous performance criteria as of March 24, 2003 (the completion date of the first review of the PRGF (rrangement). 
Table 5. Democratic Republic of the Congo: Prior Actions and Structural Performance Criteria and Benchmarks, 2004

\section{Prior actions}

Submission to Parliament of the draft 2004 budget law, reflecting the policy thrust defined with IMF staff and presented using the new nomenclature

Submission to Parliament of the draft anticorruption law

Adoption by the government of the 2004 draft supplementary budget

Submission to Parliament of the new customs code

\section{Structural performance criteria}

Adoption by the Central Bank of the Congo of the list of commercial banks to be liquidated or reorganized

Selection of an international firm to conduct the external audit of the diamond company MIBA

\section{Structural benchmarks}

Submission to Parliament of the draft law on the harmonized classification system, reducing the number of taxes collected by DGRAD

Full implementation of the new expenditure procedures, from commitment through to payment

Submission to Parliament of the new customs code

Selection of an international firm to conduct the external audit of MIBA

Finalization of the plans for reorganizing commercial banks considered viable and putting into liquidation nonviable commercial banks

Finalization of the COHYDRO reorganization plan
January 2004

Done

June 2004

June 2004

June 2004

March 2004

Done

September 2004

February 2004

Done

February 2004

Done

March 2004

1

March 2004

2

June 2004

Done

June 2004

September 2004 
Table 5. Democratic Republic of the Congo: Prior Actions and

Structural Performance Criteria and Benchmarks, 2004

Measures

Timetable

Status of

Implementation

Completion of the strategic audit of the Public

June 2004

September 2004

Enterprise Council (Conseil Supérieur du

Portefeuille)

Adoption of a simplified double-entry government

June 2004

Done accounting framework

Finalization of the Law Governing Public Institutions

December 2004

Done and the Law Governing Divestment by the State of Public Enterprises

Reorganization of the procedures for paying civil

December 2004 servants based on the recommendations of the external audit of the payroll system

${ }^{1}$ Changed into a prior action.

${ }^{2}$ Changed into a structural performance criterion for end-September. 


\title{
DEMOCRATIC REPUBLIC OF THE CONGO
}

\author{
Technical Memorandum of Understanding
}

Kinshasa, June 24, 2004

1. This memorandum covers the agreements on monitoring the implementation of the program supported by the Poverty Reduction and Growth Facility (PRGF) of the International Monetary Fund (IMF). It establishes the information to be reported and the deadlines for its submission to the IMF staff for program monitoring. It defines the quantitative performance criteria and benchmarks, as well as the structural performance criteria and benchmarks presented in the memorandum on economic and financial policies (MEFP) of the government of the Democratic Republic of the Congo (DRC), which is attached to the letter of June 24, 2004 to the Managing Director of the International Monetary Fund.

\section{A. Monitoring Program Implementation}

2. Implementation of the program covering the period April 1, 2002-July 31, 2005 will be monitored on the basis of the performance criteria and benchmarks described in paragraphs 81 and 82 and Tables 4 and 5 of the MEFP of June 24, 2004.

\section{B. Definition of Quantitative Performance Criteria and Indicators}

3. The quantitative performance criteria and benchmarks described in Table 4 of the MEFP are as follows:

a. floor on net foreign assets of the central bank (BCC);

b. ceiling on net domestic assets of the BCC;

c. ceiling on net bank credit to the government;

d. ceiling on $\mathrm{BCC}$ credit to nonfinancial public sector enterprises;

e. ceiling on $\mathrm{BCC}$ credit to the nonfinancial private sector;

f. ceiling on new nonconcessional external debt contracted or guaranteed by the government or the BCC, with maturities of more than one year, except borrowing for debt rescheduling purposes, and IMF credit;

g. ceiling on new nonconcessional external debt contracted or guaranteed by the government or the $\mathrm{BCC}$, with maturities of one year or less, except borrowing for debt rescheduling purposes, IMF credit, and normal import credits (suppliers' credits), excluding petroleum imports; and

h. ceiling on wage arrears (including all forms of compensation) for the civil service (civilian and military) and the BCC. 
The following quantitative criteria will be monitored on a continuous basis:

i. the BCC will not finance government expenditure that has not been authorized in advance by the Ministry of Finance;

j. the BCC will make no purchase of Congo franc banknotes or foreign currency in the market at a premium against payment in bank money; and

k. the government will not accumulate external payments arrears on debt service for which a debt rescheduling agreement has been concluded with the government's creditors, or on any new borrowing.

\section{Definitions}

4. Net foreign assets of the BCC are defined as the difference between the BCC's gross foreign assets and all its external obligations, as shown in the "Integrated Monetary Survey" prepared by the BCC. The net foreign assets and all the foreign currency accounts of the BCC, as well as the Integrated Monetary Survey, will be valued at the program exchange rates, which are as follows: SDR $1=\mathrm{US} \$ 1.26537$; US $\$ 1=$ CGF 313.6; and EUR $1=$ CGF 357.62.

5. The net domestic assets of the BCC are equal to the sum of the following line items, as they appear in the $\mathrm{BCC}$ balance sheet:
a. net claims on the government;
b. claims on nonfinancial public enterprises;
c. claims on the nonfinancial private sector;
d. claims on banks (net of billets de trésorerie obtained by deposit money banks);
e. claims on other banking and nonbank institutions; and
f. " "other items net," defined as other assets minus other liabilities (including capital and valuation accounts, and billets de trésorerie obtained by the public).

6. Net banking system credit to the government is defined as the sum of net claims of the central bank and of deposit money banks on the government, as defined in the "Integrated Monetary Survey" prepared by the BCC (excluding deposits linked to project-related assistance), plus the BCC's net cash deficit.

7. Fifty percent of any surplus (shortfall) over (under) the programmed amount of external budgetary assistance (excluding project assistance), net of debt service and including external debt service rescheduling, that has not been used to finance poverty-reducing expenditure, public enterprise restructuring, and domestic debt repayment (limited to crossarrears certified by the World Bank staff) will be used to reduce (increase) net banking system credit to the government, and the corresponding performance criterion will be adjusted downward (upward) accordingly. The criteria on net foreign assets and net domestic assets will be adjusted upward (downward) and downward (upward), respectively, by the 
same amount. However, the criterion regarding net foreign assets will be adjusted downward without letting the stock of net foreign assets fall below the level achieved at endDecember 2002. Expenditures related to the National Disarmament, Demobilization, and Reintegration Program (DDR), elections, civil service retirements, and domestic debt are considered projects. This adjustment does not apply to HIPC resources, which will be deposited in a special account at the BCC. The procedure for using this account is described in the Fund staff report on the decision point under the HIPC Initiative (7/4/2003).

8. BCC credit to nonfinancial public sector enterprises is equal to BCC claims on nonfinancial public enterprises, as defined in the "Integrated Monetary Survey" prepared by the BCC.

9. BCC credit to nonfinancial private sector enterprises (excluding loans to BCC personnel and advances on orders of goods and services) is equal to BCC claims on nonfinancial private enterprises, as defined in the "Integrated Monetary Survey" prepared by the BCC.

10. Wage arrears are defined as validated personnel expenses not paid for more than 30 days. Wages include all compensation paid to employees (civil service personnel, including the military, national police, members of Cabinet, and BCC staff), including bonuses and allowances. Under the program, these arrears will be assessed cumulatively and partly based on the balances of the accounts of the provincial delegated payment authorization officers (ODs) in the Treasury's general account at the BCC.

11. The government will not accumulate any payments arrears on external debt, except on debt being rescheduled with creditors.

12. The definition of external debt can be found in Decision No. 6230-(79/140), para. 9, amended on August 24, 2000 (Annex I).

13. The grant element of borrowing will be calculated on the basis of currency-specific rates based on the OECD commercial interest reference rates (CIRR) on the date the contract is signed, as specified in the Annex. A loan is defined as concessional if, on the date the contract is signed, the ratio of the present value of the loan, calculated on the basis of the reference interest rate, to its nominal value is less than 65 percent (i.e., including a grant element of at least 35 percent).

14. Base money is defined as the sum of the following:
a. currency in circulation (in and outside banks);
b. deposits of banks with the BCC;
c. deposits of public enterprises with the $\mathrm{BCC}$;
d. deposits of private enterprises and individuals with the BCC; and
e. deposits of other financial institutions, other than deposit money banks, with the BCC.




\section{Note: "Base money" excludes all billets de trésorerie issued by the BCC.}

15. The following concepts are used in the letter of intent and the memorandum on economic and financial policies:

a. Budget: annual law authorizing the government's financial operations. Transfers to the provinces are included, but the provinces' own revenues are not covered. The social security system is not consolidated in the budget;

b. Special budgets (budgets pour ordre): autonomous agencies and entities receiving earmarked revenues that, like their expenditures, are covered in the budget;

c. Extrabudgetary accounts: accounts receiving government revenue not tracked by the Treasury Management and Payment Authorization Directorate. The consolidation of these accounts with those that are regularly monitored by the Treasury Management and Payment Authorization Directorate is necessary for a complete picture of budget execution; and

d. Poverty-reduction expenditure: "pro-poor" spending as defined in the new nomenclature on the basis of the priorities set forth in the I-PRSP and detailed in Annex II.

\section{Structural Performance Criteria and Benchmarks}

16. The structural performance criteria and benchmarks are described in Table 5 of the memorandum on economic and financial policies.

\section{Reporting}

17. The authorities will forward to the IMF's African Department, as soon as possible and preferably by e-mail or fax, the data and information needed to monitor program implementation. These data and information must be duly reconciled so as to ensure their internal consistency. Following are the data or documents to be submitted:

\section{Exchange system}

a. Volume of purchases and sales of foreign exchange on the interbank market, between commercial banks and their customers, and by exchange bureaus;

b. Volume of purchases and sales (interventions) by the BCC on the interbank market;

c. Average Congo franc/U.S. dollar reference exchange rate of the BCC (indicative rate);

d. Average Congo franc/U.S. dollar exchange rate on the interbank market;

e. Average Congo franc/U.S. dollar exchange rate offered by commercial banks to their customers; and,

f. Average Congo franc/U.S. dollar exchange rate used by exchange bureaus. 
Note: The above information is to be submitted with a time lag of one day.

\section{Banking system}

a. Integrated monetary survey, with a breakdown into domestic currency and foreign currency;

b. Monetary survey of the BCC, with a breakdown into domestic currency and foreign currency;

c. BCC operating account;

d. BCC investment budget;

e. Implementation of the BCC's cash flow plan;

f. Statement of wage arrears owed to BCC staff;

g. Monetary survey of deposit money banks, with a breakdown into domestic currency and foreign currency;

h. Net banking system credit to the government;

i. $\quad$ Net banking system credit to public sector enterprises;

j. $\quad$ Structure of nominal and real interest rates of deposit money banks;

k. Reserves (voluntary and required) of deposit money banks;

1. Structure of BCC interest rates;

m. Structure of billets de trésorerie rates; and

n. Premium on Congo franc banknotes purchased in the market against bank money.

\section{Note: The above monthly information is to be submitted not later than three weeks after the end of each month.}

\section{Public sector}
a. Implementation of Treasury cash flow plan;
b. Expenditure execution by type and by ministry/institution;
c. Validated wage bill by category of payee, region (Kinshasa/provinces), and activity status (active/retired);
d. Wage bill debited from the Treasury General Account by category of payee, region, and activity status;
e. Paid wage bill by category of payee, region, and activity status;
f. Paid employees, by category of payee, region, and activity status;
g. Civil service pay scale (if changed);
h. Issues, redemptions, and stocks of treasury bills (including maturity and interest charges), by category of creditor (commercial banks, public enterprises, and other);
i. Public sector domestic debt, by category of creditor (commercial banks, private entities, etc.): collect and report data related to domestic public debt as soon as they are available; and
j. Payments arrears on centralized expenditures. 


\section{Note: The above information is to be submitted not later than three weeks after the end of each month.}

Starting in August 2004, and following implementation of the new expenditure procedures, the budget tracking statements mentioned in Annex III will also be forwarded

\section{Real sector}

Report as soon as possible indicators on recent economic developments and other related data, such as the consumer price index, once a week; merchandise exports (in value and volume) of crude oil, copper, cobalt and zinc, and industrial and artisanal diamonds; imports (in value and volume), if possible by principal product and showing petroleum products separately; and output indicators of the manufacturing, mining, and services sectors, published in the BCC's monthly reports on economic activity. Monthly tax base (imports) prepared by the Customs and Exercise Office (OFIDA).

\section{External debt}

a. Actual disbursements of external assistance, whether or not to finance projects, including those associated with new contracted loans (on a monthly basis, with a lag of three weeks);

b. Monthly breakdown by interest and principal, and classification by creditor, of debt service payments made;

c. Composition of monthly external debt-service obligations, by maturity (including after debt rescheduling by the Paris Club, other bilateral creditors, and multilateral creditors, commercial debt, and short-term debt), and the stock of external arrears, taking into account actual payments, with a breakdown by principal and interest, and classification by creditor (to be provided quarterly by the Public Debt Management Office (OGEDEP)); and

d. Copies of the debt rescheduling agreements with the Paris Club, non-Paris Club bilateral creditors, commercial creditors, and multilateral creditors, as soon as such agreements have been concluded. Also, all individual loan information is essential for the debt sustainability analysis in the context of the HIPC Initiative, and also for debt management purposes during the interim period.

\section{Note: The above monthly information is to be provided three weeks after the end of each month.}

A progress report on implementation of the structural reforms will be submitted to Fund staff each month. In addition, information on the legal and regulatory environment as it affects the business environment (new decrees, circulars, and laws) and price policy, as well as the official gazette, will also be reported to Fund staff. 
Jean-Claude Masangu Mulongo Governor

Central Bank of the Congo
M.F. Muamba Tshishimbi

Minister of Budget
André-Philippe Futa

Minister of Finance

Kinshasa, June 24, 2004 


\section{Definition of External Debt}

1. The definitions of "debt" and "concessional borrowing" for the purposes of this memorandum of understanding are as follows:

(a) As set out in Point 9 of the Guidelines on Performance Criteria with Respect to Foreign Borrowing adopted by the IMF's Executive Board on August 24, 2000, debt is understood to mean a current, that is, not contingent, liability created under a contractual agreement through the provision of value in the form of assets (including currency) or services, and which requires the obligor to make one or more payments in the form of assets (including currency) or services at some future points in time; these payments will discharge the principal and/or interest liabilities incurred under the contract. Debt can take a number of forms, the primary ones being as follows: (i) loans, that is, advances of money to the obligor by the lender on the basis of an undertaking that the obligor will repay the funds in the future (including deposits, bonds, debentures, commercial loans, and buyers' credits) and temporary exchanges of assets that are equivalent to fully collateralized loans, under which the obligor is required to repay the funds, and usually pay interest, by repurchasing the collateral from the buyer in the future (such as repurchase agreements and official swap arrangements); (ii) suppliers' credits, that is, contracts where the supplier permits the obligor to defer payment until some time after the date on which the goods are delivered or services are provided; and (iii) leases, that is, arrangements under which property is provided that the lessee has the right to use for one or more specified period(s) of time, which are usually shorter than the total expected service life of the property, while the lessor retains the title to the property. For the purpose of this guideline, the debt is the present value (at the inception of the lease) of all lease payments expected to be made during the period of the arrangement, excluding those payments that cover the operation, repair, or maintenance of the property. Under the definition of debt set out above, arrears, penalties, and judicially awarded damages arising from failure to make payment under a contractual obligation that constitutes debt are debt. Failure to make payment on an obligation that is not considered debt under this definition (e.g., payment on delivery) will not give rise to debt.

(b) A loan is considered concessional if, on the date the contract is signed, the ratio of the present value of the loan, based on the reference interest rates to the nominal value of the loan is less than 65 percent (i.e., a grant element exceeding 35 percent). The reference interest rates used in this assessment are the commercial interest reference rates (CIRRs) established by the Organization for Economic Cooperation and Development (OECD). For debts with a maturity exceeding 15 years, the ten-year reference interest rate published by the OECD is used to calculate the grant element. For shorter maturities, the six-month market reference rate is used. 


\section{Definition of Poverty-Reducing Spending}

\section{The concept}

Poverty-reducing spending comprises all actions by the government for the good and well-being of the people, in the spirit of the priorities set out in the Interim Poverty Reduction Strategy Paper.

\section{Criteria}

To identify poverty-reducing spending in the budget, the government has based its choices on the classification by the general functions of government defined as targets in favor of the people.

From this point of view, spending on the following functions and sub-functions shall be considered to be poverty-reducing spending:

02 Defense

02. 30 Reintegration of demobilized soldiers

03 Security

03. 20 Courts

04 Economic Affairs

04. 21 Agriculture, livestock

04. 22 Forestry

04. 23 Hunting and fishing

04. 24 Rural development

04. 32 Electricity (in the provinces)

04. 51 Roads

04. 52 Sea, river and lake transport

04. 53 Railroads

05 Environmental protection

05. 11 Waste management

05. 30 Sanitation

05. 40 Pollution control

05. 50 Protection of fauna and flora

06 Public Housing

06. 10 Development of housing

06. 20 Development of community facilities

06. 30 Water supply

06. $40 \quad$ Electricity supply 


\section{$07 \quad$ Health}

07. 10 Medicines, prostheses, and medical equipment and supplies

07. 20 Local medical services

07. 30 Hospital services

07. $40 \quad$ Public health services

07. 50 Research and development in the field of health

\section{Education}

09. 10 Basic Education

09. 20 Secondary Education

09. 30 Higher education (only equipment and rehabilitation of infrastructure)

09. 70 Other educational matters (only SECOPE: Wages of Primary and Secondary School Teachers)

09. 80 Continuing professional education

10 Welfare

10. 40 Targeted protection

10. 50 Unemployment programs

10. 60 Housing and housing conditions

10. 70 Social exclusion programs 


\section{Budget-Tracking Statements}

\section{Statement 1: Main budget-tracking statement. Monthly, starting in August 2004.}

This statement describes expenditures according to the four phases of the expenditure chain (commitment, verification, payment order, payment), on the one hand, and by type of expenditure, on the other, and cumulatively from the start of the fiscal year.

This statement should also have two intermediate columns for payment authorizations sent to the $\mathrm{BCC}$ and payment authorizations pending transmission to the $\mathrm{BCC}$.

A specific column for automatic payments (décaissements d'office) will also be placed next to the column for payment orders.

The last column of the main budget tracking statement is the "Balances Outstanding" column, which is the difference between payment orders signed by the responsible payment authorizing officer and actual payments by the BCC (not the difference between payment authorizations sent to the $\mathrm{BCC}$ and actual payments by the $\mathrm{BCC}$ ).

\section{Statement 2: Main budget-tracking statement by administrative classification. Monthly,} starting in August 2004.

Based on the main statement, this document will present expenditures by administrative classification (2003 revised nomenclature rather than classification by type). Additionally, the statement will keep expenditures initiated by, and earmarked for, the Offices of Ministers (Cabinets) separate from those initiated by, and earmarked for, the administrations.

\section{Statement 3: Main budget-tracking statement by geographical distribution. Monthly, starting in August 2004.}

Based on the balances of the main statement, this document will present expenditures by type, distinguishing between expenditures in Kinshasa and those in the provinces.

Computer tools and training permitting, separate service codes will be assigned for Kinshasa and for each province; this will permit tracking of the distribution of expenditures among the ten provinces and Kinshasa.

\section{Statement 4: Main budget-tracking statement, "Poverty-Reducing Expenditures." Monthly, starting in August 2004.}

Based on Statement 2, expenditures will be presented by type, with one line indicating the share of expenditures identified as poverty-reducing expenditures.

\section{Statement 5: Main budget-tracking statement, "Major Government Functions." Monthly, starting in August 2004.}

Based on Statement 2, this document will present expenditures by major government functions (as defined in the 2002 revised nomenclature). 
Democratic Republic of the Congo: Relations with the Fund (As of May 31, 2004)

I. Membership Status: Joined: September 28, 1963

II. General Resources Account:

Quota

Fund holdings of currency

III. SDR Department:

Net cumulative allocation

Holdings

IV. Outstanding Purchases and Loans:

Poverty Reduction and Growth Facility (PRGF) arrangement

Article VIII

SDR Million

533.00

533.00

SDR Million

86.31

5.03

SDR Million

500.07
In Percent of

Quota

100.00

100.00

In Percent of

Allocation

100.00

5.82

In Percent of

Quota

93.82

\section{Latest Financial Arrangements:}

$\begin{array}{lcccc}\text { Type } & \begin{array}{c}\text { Approval } \\ \text { Date }\end{array} & \begin{array}{c}\text { Expiration } \\ \text { Date }\end{array} & \begin{array}{c}\text { Amount Approved } \\ \text { (SDR Million) }\end{array} & \begin{array}{c}\text { Amount Drawn } \\ \text { (SDR Million) }\end{array} \\ \text { PRGF } & \text { June 12, 2002 } & \text { June 11, 2005 } & 580.00 & 500.07 \\ \text { Stand-By } & \text { June 9, 1989 } & \text { June 8, 1990 } & 116.40 & 75.00 \\ \text { SAF } & \text { May 15, 1987 } & \text { May 14, 1990 } & 203.70 & 145.50\end{array}$

VI. Projected Payments to Fund (without HIPC Assistance)

(In SDR millions; based on existing use of resources and present holdings of SDRs):

Principal

Charges/interest

Total

\begin{tabular}{lrrrr}
\multicolumn{5}{c}{ Forthcoming } \\
\hline $\mathbf{2 0 0 4}$ & $\mathbf{2 0 0 5}$ & $\mathbf{2 0 0 6}$ & $\mathbf{2 0 0 7}$ & $\mathbf{2 0 0 8}$ \\
0.00 & 0.00 & 0.00 & 42.00 & 86.67 \\
3.44 & 3.82 & 3.84 & 3.81 & 3.48 \\
3.44 & 3.82 & 3.84 & 45.81 & 90.15
\end{tabular}

VII. Projected Payments to Fund (with Board-approved HIPC Assistance)

(In SDR millions; based on existing use of resources and present holdings of SDRs):

Principal

Charges/interest

Total

\begin{tabular}{rrrrr}
\multicolumn{5}{c}{ Forthcoming } \\
\hline $\mathbf{2 0 0 4}$ & $\mathbf{2 0 0 5}$ & $\mathbf{2 0 0 6}$ & $\mathbf{2 0 0 7}$ & $\mathbf{2 0 0 8}$ \\
0.00 & 0.00 & 0.00 & 42.00 & 86.67 \\
2.87 & 3.82 & 3.84 & 3.81 & 3.48 \\
2.87 & 3.82 & 3.84 & 45.81 & 90.15
\end{tabular}




\section{Implementation of HIPC Initiative:}

I. Commitment of HIPC assistance

Decision point date

Framework

Assistance committed by all creditors (millions U.S. dollars)

July 2003

Of which: IMF assistance (millions U.S. dollars)

$6,311.00$

(SDR equivalent millions)

318.90

228.30

Completion point date

Floating

II. Disbursement of IMF assistance (millions SDRs)

Assistance disbursed to the member

Interim assistance

Completion point balance

Additional disbursement of interest income

\section{Total disbursements}

\section{Exchange Rate Arrangement:}

The Democratic Republic of the Congo's (DRC) currency is the Congo franc, which, since May 26, 2001, has been freely floating. On May 31, 2004, the rate was US\$1=CGF 386.69. From July 1, 1998 through May 25, 2001, a multiple exchange rate system was in effect, implying an official rate, the most recent being US $\$ 1=\mathrm{CGF} 50$, and a rate determined in the parallel market.

Effective February 10, 2003 the DRC has accepted the obligations under Article VIII, Sections $2(a)$, 3, and 4 of the Fund's Articles of Agreement. However, the DRC maintains measures that give rise to one restriction and one multiple currency practice (MCP) subject to Fund approval under Article VIII of the Fund's Articles of Agreement. The exchange restriction involves an outstanding net debit position vis-à-vis other contracting members under the inoperative regional payments agreement with the Economic Community of the Great Lakes Countries (CEPGL). The multiple currency practice involves a fixed exchange rate set on a quarterly basis applying to transactions through the bilateral payments agreement (BPA) with Zimbabwe. On December 24, 2003, the Executive Board approved an extension until December 31, 2004 of the period to phase out remaining exchange restrictions subject to approval under Article VIII.

\section{Last Article IV Consultation:}

(a) Consultations with the Democratic Republic of the Congo are held in accordance with the provisions of the decision on consultation cycles approved on July 15, 2002.

(b) The last Article IV consultation was concluded by the Executive Board on March 24, 2003 (Country Report No. 03/161).

\section{Safeguards Assessment}

Under the IMF's safeguards assessment policy, the BCC is subject to an assessment with respect to the PRGF arrangement, which was approved on June 12, 2002 and is scheduled to expire on June 11, 2005. A safeguards assessment of the BCC was completed on January 3, 2003. The assessment 
concluded that substantial risks of misreporting or misuse may exist due to vulnerabilities in the external audit mechanism, financial reporting framework, and system internal controls. Staff findings, proposed recommendations under program conditionality and other recommendations are reported in (Country Report No. 03/161). Implementation of the measures by the BCC continues to be monitored by staff.

\section{Technical Assistance:}

\section{Subject}

Monetary statistics

Exchange rate system

MFD

Article VIII obligations

Capacity-building assistance to the central bank

Monetary policy/ foreign exchange management, bank supervision and budget

MFD

\section{Department Staff Member}

Mr. Papadacci

Ms. Tanase

Mr. Bussers

Ms. De Boeck

Mr. Leimone

Mr. Laurens

Mr. Sarr

Mr. Akitoby

Mr. Paul

Mr. Bussers

Mr. Tavernier

Mr. Paul

Mr. Beaumé

Mr. Laurens

Mr. Sarr

Mr. Paul

Mr. Beaumé

Mr. Régnard

Mr. Tavernier

\section{Date}

Jun. 29-Jul. 12, 2000

May 9-14, 2001

Jul. 1-7, 2001

Oct. 21-29, 2002

Oct. 12-26, 2001

Aug. 8-Sep. 20, 2002

Feb. 1-7, 2002

Jul. 15-25, 2002

Oct. 29-Nov. 12, 2002 
Action plan for developing the financial system in the DRC

Tax administration/ policy

Tax policy

Expenditure

management

Revenue administration

FAD

Real sector and government finance statistics

Government finance statistics

FAD

FAD

FAD

STA
MFD

Mr. Laurens

Nov. 5-15, 2003

Mr. Fonteyne

Ms. Nkhata

Mr. Saffré

Mr. Paul

Mr. Regnard

Mr. Vandecan

Mr. Pauwels

Mr. Corfmat

Mr. Fossat

Ms. Geourjon

Mr. Laporte

Mr. Schneider

Sep.4-25, 2002
Mr. Schiller

Mr. Fournel

Mr. Barrier

Mr. Bouley

Mr. Calcoen

Mr. Bouley

Mr. Lepage

Mr. Kwant

Mr. Benon

Mr. Jolibert

Mr. Montagnard-Rentier

Mr. Benon

Jul. 13-26, 2003

Mr. Lesprit

Mr. Benon

Mr. Marie

Mr. Gorter

Mr. Maiga

Mar. 19-Apr. 13, 2002

Aug. 4-18, 2001

Aug. 31-Sep. 10, 2002

Nov. 11-25, 2003

Dec. 3-17, 2002

Sep. 16-20, 2003

Jun. 4-13, 2001 
Multisector statistics

STA

Ms. Fisher

Oct. $8-21,2003$

Mr. Tanase

Ms. Noukovska

Mr. Fiévet

Mr. Maiga

Mr. Ntungwanayo (World

Bank)

XIII. Long-Term Resident Experts:

Expenditure management

Customs administration

Tax administration

Advisor to Governor of $\mathrm{BCC}$

\author{
Mr. Catalan \\ Mr. Bremeersch \\ Mr. Schlotterbeck \\ Mr. Raouya \\ Mr. d'Ambrières \\ Mr. Nyssens
}

Jan. 2002-Jun. 2004

Jan. 2002-Jan. 2004

Jan. 2002-Mar. 2003

Since Sept. 2003

Apr. 2002-Dec. 2003

Since January 2004

IV. Resident Representative: Mr. Gons was Acting Resident Representative from July 20 to September 20, 2002. Mr. Kouwenaar assumed his duties as Senior Resident Representative on September 21, 2002. 


\section{Democratic Republic of the Congo \\ Relations with the World Bank Group ${ }^{1}$}

\section{Introduction}

1. This annex first underscores the importance that the Government of the Democratic Republic of the Congo (DRC) attaches to effective partnership with external creditors, donors, and foreign investors. It then summarizes the strategy and activities of the World Bank Group (IDA, IFC, and MIGA) in the DRC. It concludes with a description of areas of specific collaboration between the World Bank Group and the International Monetary Fund.

\section{Partnership in the DRC's development strategy}

2. The DRC was cut off from international financial assistance from 1993 until 2001, primarily due to economic and financial mismanagement, conflict and war. From 1997-2001, the conflict and its socioeconomic impact led to over three million deaths, most of which were due to widespread disease and hunger, as well as extensive destruction of infrastructure and the collapse of institutions.

3. When President Joseph Kabila took office following his father's death in January 2001, he sought to reestablish relations with international financial institutions. The International Monetary Fund responded with a Staff-Monitored Program (SMP), covering the period June 1, 2001-March 31, 2002, and in July 2001 IDA approved a Transitional Support Strategy (TSS), initially underpinned by a US\$50 million grant for an Emergency Early Recovery Project (EERP).

4. Progress towards peace and good performance under the SMP and the EERP has led to substantial financial reengagement by multilateral and bilateral institutions. The Interim PRSP (I-PRSP) was discussed by the IDA Board on June 11, 2002. Following clearance of arrears to the IMF, a three-year PRGF and the HIPC preliminary document were approved, and the I-PRSP discussed, by IMF Executive Directors on June 12, 2002. IDA's Economic Recovery Credit (ERC), approved by the IDA Board on June 13, 2002, became effective following arrears clearance on July 3, 2002. The Paris Club granted significant relief on bilateral debt at its September 2002 meeting. The first review of the PRGF was completed on March 24, 2003 at the same time as the IMF's 2002 Article IV consultation. The decision point under the enhanced HIPC Initiative was approved by IMF Executive Directors on July 23, 2003 (at the same time as the conclusion of the second review under the PRGF), and by

\footnotetext{
${ }^{1}$ This document was approved by the Africa Region ROC on June 23, 2004. Questions should be addressed to Brendan Horton (473-5587), Eric Nelson (473-6699), or Keiko Kubota (473-6836) at the World Bank.
} 
IDA Executive Directors on July 24, 2003. In December 2003 at the Consultative Group meeting in Paris, donors pledged US\$3.9 billion in financial assistance to the DRC over the next few years. The third PRGF review was concluded by the IMF Board on March 3, 2004.

5. The preparation of the PRSP is now a key focus of government partnership efforts and the object of close collaboration between IMF and IDA staffs. Following the preparation of the I-PRSP in June 2002, the first annual Preparation Status Report and related JSA have been produced, and discussed by the IMF and IDA Boards. The authorities plan to complete preparation of the full PRSP by August 2005.

\section{Bank Group strategy and operations (IDA, IFC and MIGA) ${ }^{2}$}

6. Overall strategy. On July 31, 2001, the Executive Directors of IDA endorsed a Transitional Support Strategy, to map out Bank financial and nonfinancial support to the DRC for the following 12-24 months, which included the Emergency Early Recovery Project (EERP), the Economic Recovery Credit (ERC), the Private Sector Development and Competitiveness Project (PSDC), and the Emergency Multisector Rehabilitation and Reconstruction Project (EMRRP) (as described hereunder). A new TSS was approved by the Board on February 26, 2004 and covers the period 2004-06.

7. The new TSS sets forth the scope and configuration of future support. The TSS accompanies the government's efforts during the transition period, with a particular focus on: (i) social stability and security; (ii) high and shared growth; (iii) governance and institutional strengthening; and (iv) social development. It supports the following projects:

- FY04: Emergency Economic and Social Reunification Support Project; Southern Africa Power Pool Project; HIV/AIDS Project; and Post-Reunification Economic Recovery Credit;

- FY05: Emergency Demobilization and Reintegration Project; Health Sector Rehabilitation Project; Education Sector Rehabilitation Project; Emergency Living Conditions Project; Emergency Social Action Program; and an adjustment operation; and

- FY06: a Multi-Sector Transport Project; a Public Utilities Project; an Agriculture Project; a Private Sector Development/Financial Sector Development Project; and an adjustment operation.

\footnotetext{
2 The Bank reopened a country office in Kinshasa, and a country manager assumed his post in January 2002.
} 


\section{Existing support}

\section{IDA}

8. IDA provides support through Trust Funds, fast disbursing credits, and investment operations. Operational details are summarized in Table 1.

\section{Trust funds}

9. Since October 2000, the first Emergency Trust Fund has financed the Emergency Stabilization and Recovery Project to help communities via projects in the health, education, food security, forestry, water supply (rural and peri-urban areas), and rural roads rehabilitation sectors, to support street/abandoned children and capacity building, and to assist the government in implementing its economic reform agenda, by conducting a number of key reform studies, and coordinating donor assistance. IDA is administering this US\$13.540 million trust fund on behalf of Belgium, Canada, and the Netherlands. The FED committee of the European Union has recently provided a new contribution of 9.5 million Euros for Capacity Building Support to the DRC, which includes support to the Ministry of Finance and Central Bank of the Congo (BCC), and a set of line ministries.

\section{Grants and credits}

\section{Fast disbursing policy reform operations}

10. The Bank is providing support to policy reform operations - the ERC and the Post Reunification Economic Recovery Credit (PRERC). The ERC, approved in 2002 in the amount of US\$450 million, provided support to the arrears clearance process (US\$338 million) and other balance of payments support (US\$72 million), support to a voluntary departures program at GECAMINES, the national copper mining company (US\$25 million); and forestry sector reforms (US\$15 million). The operation was satisfactorily implemented and closed in June 2003. The PRERC, approved in February 2004, provides balance of payments support (US\$85 million), support to civil service retirement reform (US\$47.5 million), domestic debt settlement (US\$42.5 million), and utilities billing and payment reform (US\$25 million). Conditionalities involve the adoption of adequate reform strategies plus fiduciary arrangements to ensure that funds reach intended recipients. It is expected that disbursements will occur before June 2005.

\section{Traditional investment operations}

11. The Bank is providing support through investment operations in the DRC in the areas of (i) infrastructure rehabilitation - the EERP, the EMRRP, and the Emergency Economic and Social Reunification Support Project; (ii) demobilization and reintegration - the Emergency Demobilization and Reintegration Project (EDRP); (iii) private sector development - the Private Sector Development and Competitiveness Project (PSDC); (iv) the 
Southern Africa Power Market Project (SAPM); and (v) HIV/AIDS (Multi-Sectoral HIV/AIDS Program). Implementation of all projects is satisfactory.

12. With respect to infrastructure rehabilitation, the EERP rehabilitated the MatadiKinshasa road, and improved the security of the blood supply to prevent transmission of HIV/AIDS. It has been fully disbursed. In the case of the EMRRP, disbursements are somewhat slower than initially programmed because of the need to properly prepare rehabilitation programs (infrastructure is extremely dilapidated). It is now expected that some 80 percent of the total credit will be committed by end 2004. The Emergency Economic and Social Reunification Support Project focuses on infrastructure rehabilitation in the reunified provinces, and 25 percent of the credit has been disbursed.

13. With respect to demobilization and reintegration, the recently approved Emergency Demobilization and Reintegration Project provides support to the demobilization and reintegration of 150,000 ex-combatants country-wide. The project was developed within the framework of the Multi-Country Demobilization and Reintegration Program (MDRP) for the Great Lakes region, a regional partnership initiative comprising seven governments and over 40 donor and multilateral agencies. In a recent meeting of MDRP partners, donors endorsed a US $\$ 100$ million MDRP Trust Fund grant (cofinancing) to complement the IDA grant financing. Collaboration in the field between the Bank and other donors and multilateral agencies is proceeding well, and IDA is paying particular attention to the deepening of cooperation.

14. With respect to private sector development, the PSDC is helping reform inefficient public enterprises and improving the investment climate, to attract private investment, which would contribute to economic growth.

15. With respect to electric power, the Southern Africa Power Market Project (SAPM) will strengthen the capacity of the Coordination Center of the Southern Africa Power Pool (SAPP) to promote and manage electricity trade in the region; remove transmission bottlenecks; and connect member countries to the regional grid, which will foster economic growth. The DRC component is the largest, and will enable the DRC to supply electricity to neighboring countries. It was approved in November 2003.

16. With respect to HIV/AIDS, the recently approved Multi-Sectoral HIV/AIDS program helps mitigate the negative impact of HIV/AIDS on the socioeconomic development of the DRC. 
Table 1: Summary of World Bank Financial Assistance

\begin{tabular}{|c|c|c|c|c|}
\hline Credit Name & Amount & Date approved & Theme & Status \\
\hline $\begin{array}{l}\text { Emergency Early } \\
\text { Recovery Project } \\
\text { (EERP) }\end{array}$ & $\begin{array}{l}\text { US\$50 } \\
\text { million IDA } \\
\text { grant }\end{array}$ & Jul. 31, 2001 & $\begin{array}{l}\text { Economic reforms, a pilot CDD initiative, } \\
\text { rehabilitation of the Kinshasa-Matadi road, } \\
\text { and HIV/AIDS activities }\end{array}$ & $\begin{array}{l}\text { Works } \\
\text { completed, } \\
\text { fully disbursed }\end{array}$ \\
\hline $\begin{array}{l}\text { Economic Recovery } \\
\text { Credit (ERC) }\end{array}$ & $\begin{array}{l}\text { US\$450 } \\
\text { million }\end{array}$ & Jun. 13, 2002 & $\begin{array}{l}\text { Budgetary/foreign exchange support, and } \\
\text { forestry and mining sector reforms }\end{array}$ & $\begin{array}{l}\text { Completed and } \\
\text { fully disbursed }\end{array}$ \\
\hline $\begin{array}{l}\text { Emergency Multi- } \\
\text { Sector Rehabilitation } \\
\text { and Reconstruction } \\
\text { Project (EMRRP) }\end{array}$ & $\begin{array}{l}\text { US\$454 } \\
\text { million, o/w } \\
\text { US\$44 } \\
\text { million grant }\end{array}$ & Aug. 6, 2002 & $\begin{array}{l}\text { Reconstruction and rehabilitation of critical } \\
\text { infrastructure, increase in service delivery, } \\
\text { institutional capacity strengthening, } \\
\text { development of sectoral strategies }\end{array}$ & $\begin{array}{l}\text { About } 80 \\
\text { percent of } \\
\text { credit funds } \\
\text { will be } \\
\text { committed by } \\
\text { end of CY04 }\end{array}$ \\
\hline $\begin{array}{l}\text { Private Sector } \\
\text { Development and } \\
\text { Competitiveness } \\
\text { Project (PSDC) }\end{array}$ & $\begin{array}{l}\text { US\$120 } \\
\text { million }\end{array}$ & Jul. 29, 2003 & $\begin{array}{l}\text { Increase competitiveness by improving the } \\
\text { investment climate, support reform of } \\
\text { public enterprises, stimulate economic } \\
\text { diversification in Katanga, and provide job } \\
\text { search support for the unemployed }\end{array}$ & Ongoing \\
\hline $\begin{array}{l}\text { Emergency Economic } \\
\text { and Social } \\
\text { Reunification Support } \\
\text { Project }\end{array}$ & $\begin{array}{l}\text { US\$214 } \\
\text { million, o/w } \\
\text { US\$164 } \\
\text { million grant }\end{array}$ & Sep. 11, 2003 & $\begin{array}{l}\text { Finance emergency rehabilitation activities } \\
\text { (large infrastructure, urban rehabilitation, } \\
\text { community development - in particular to } \\
\text { the reunified provinces) }\end{array}$ & $\begin{array}{l}25 \text { percent of } \\
\text { credit has been } \\
\text { disbursed }\end{array}$ \\
\hline $\begin{array}{l}\text { Southern Africa } \\
\text { Power Market Project } \\
\text { (SAPM) }\end{array}$ & $\begin{array}{l}\text { US\$178.6 } \\
\text { million }\end{array}$ & Nov. 11, 2003 & $\begin{array}{l}\text { Restore capacity to deliver electric power to } \\
\text { SAPP }\end{array}$ & Ongoing \\
\hline $\begin{array}{l}\text { Post-Reunification } \\
\text { Economic Recovery } \\
\text { Credit (PRERC) }\end{array}$ & $\begin{array}{l}\text { US\$200 } \\
\text { million }\end{array}$ & Feb. 26, 2004 & $\begin{array}{l}\text { Support to civil service reform retiree } \\
\text { program, repayment of government internal } \\
\text { debt to private creditors, and reform of } \\
\text { utilities billing and payment }\end{array}$ & $\begin{array}{l}\text { Credit to be } \\
\text { fully disbursed } \\
\text { between now } \\
\text { and June } 2005\end{array}$ \\
\hline $\begin{array}{l}\text { Multi-sectoral } \\
\text { HIV/AIDS program } \\
\text { (MAP) }\end{array}$ & $\begin{array}{l}\text { US\$102 } \\
\text { million }\end{array}$ & Mar. 26, 2004 & $\begin{array}{l}\text { Mitigate the negative impact of the } \\
\text { HIV/AIDS epidemic on the socioeconomic } \\
\text { development of the DRC through } \\
\text { prevention of transmission, support, and } \\
\text { care for persons living with the virus (PLV) }\end{array}$ & Ongoing \\
\hline $\begin{array}{l}\text { Emergency } \\
\text { Demobilization and } \\
\text { Reintegration Project }\end{array}$ & $\begin{array}{l}\text { US\$100 } \\
\text { million IDA } \\
\text { grant; plus } \\
\text { US\$100 } \\
\text { million in } \\
\text { counterpart } \\
\text { (MDRP) } \\
\text { funds (grants) }\end{array}$ & May 25,2004 & $\begin{array}{l}\text { Demobilize } 150,000 \text { ex-combatants } \\
\text { country-wide and help them transition to } \\
\text { civilian life, help increase social and } \\
\text { economic expenditures, and lower defense } \\
\text { expenditures }\end{array}$ & $\begin{array}{l}15 \text { percent of } \\
\text { total amount to } \\
\text { be disbursed by } \\
\text { end CY04 }\end{array}$ \\
\hline
\end{tabular}




\section{Nonlending activities}

17. For several years prior to and during the TSS, IDA assistance has also emphasized nonlending activities and advisory services, including through trust funds and grants, to improve the understanding of the socioeconomic context, rebuild the knowledge base to support policy dialogue and design effective poverty reduction strategies. FY03 activities included a Public Expenditure Review (focusing on the overall structure of expenditures; specific issues in the health, education, and transport sectors; and evaluation of the DRC's ability to monitor execution of poverty-related expenditures), strengthening of public expenditure management systems, support to the PRSP process, especially the consultation and participatory diagnostic processes. Other FY03 activities included an update of the poverty profile, and a Debt Sustainability Analysis for the HIPC program, undertaken in collaboration with the IMF. For FY04, a Country Procurement Assessment Review was undertaken, as was an Institutional Governance Review (focusing on service delivery issues); and a Forestry Sector Review. Other activities initiated in FY04, and which will continue into FY05, include an Education Sector Analysis, which will serve as the basis for discussion of a finance (priorities) strategy for the education sector; a Health Sector Analysis, which will feed into the PRSP; and preparation of an Agricultural Sector Review.

\section{IFC}

18. IFC has supported the cellular telephone operator Celtel, the local subsidiary of MSI-CIH, with a US\$7 million loan in 2002 and a further US\$20 million in 2003. IFC expects to proceed with two early mining investments, which would be among the first under the new Mining Code. In the financial sector, IFC plans to assist banks operating in the DRC with trade financing facilities, to help establish routine trade finance operations. IFC will work closely with the Bank in the context of the PSDC project to help implement specific sectoral initiatives and key investment projects. To this end, in FY05 the IFC will open an office in Kinshasa with APDF staff. IFC has also spent considerable effort in resolving outstanding disputes in the DRC, one of which, UTEXAFRICA, was settled in 2002. However, two others remain on the books: SOTEXKI, a textile business in Kisangani, in the North East, and the Grands Hotels du Congo (formerly Inter-Continental).

\section{MIGA}

19. MIGA can now issue guarantees for projects because the DRC has paid its initial capital subscription in full. Several projects are under consideration, notably in the mining, power, and telecommunications sectors.

\section{Bank-Fund collaboration in specific areas}

20. In addition to its direct assistance to the DRC, the Bank also supports policy reforms in close collaboration with the Fund in a number of areas: donor coordination, public finance management, decentralization, public enterprise reform, financial sector reform, governance and anti-corruption, and the PRSP process. Table 2 details areas of collaboration, and areas where the Bank and the Fund lead. 
Table 2: Summary of Bank-Fund Collaboration

\begin{tabular}{|c|c|c|c|}
\hline Thematic area & Areas of collaboration & Bank lead & Fund lead \\
\hline $\begin{array}{l}\text { Public finance } \\
\text { management }\end{array}$ & $\begin{array}{l}\text { Preparing proposed reforms of } \\
\text { customs and internal indirect } \\
\text { taxes, corporate taxation; and } \\
\text { reforming taxation in mining } \\
\text { and forestry sectors }\end{array}$ & $\begin{array}{l}\text { Financing technical assistance in the area } \\
\text { of training and the structure of corporate } \\
\text { taxation; conducting in depth analysis of } \\
\text { forestry taxation systems }\end{array}$ & $\begin{array}{l}\text { Technical assistance for improving tax } \\
\text { administration }\end{array}$ \\
\hline $\begin{array}{l}\text { Public enterprise } \\
\text { reform }\end{array}$ & $\begin{array}{l}\text { Collaboration on the public } \\
\text { finance aspects of these } \\
\text { reforms (e.g., cross arrears, } \\
\text { corporate tax regimes) }\end{array}$ & $\begin{array}{l}\text { Preparation and execution of reform of } \\
\text { public enterprise sector; and improving the } \\
\text { legal, regulatory, judicial and fiscal } \\
\text { environment for private sector } \\
\text { development }\end{array}$ & -- \\
\hline $\begin{array}{l}\text { Financial sector } \\
\text { reform }\end{array}$ & -- & Restructuring of commercial banks & $\begin{array}{l}\text { Monetary policy issues and central } \\
\text { bank restructuring }\end{array}$ \\
\hline $\begin{array}{l}\text { Governance and } \\
\text { anti-corruption }\end{array}$ & $\begin{array}{l}\text { Preparation of Code of } \\
\text { Conduct for Public Servants, } \\
\text { Anticorruption and Anti } \\
\text { Money-laundering Laws, }\end{array}$ & $\begin{array}{l}\text { Aiding in preparation of workshops and } \\
\text { seminars on the formulation of an overall } \\
\text { governance and anticorruption strategy; } \\
\text { providing advice and comments on } \\
\text { legislation as needed } \\
\text { Financing technical assistance for a } \\
\text { comprehensive procurement reform: } \\
\text { preparation of a Country Procurement } \\
\text { Assessment Review, production of a new } \\
\text { procurement code, the implementation of a } \\
\text { system of procurement follow up, and } \\
\text { reorganization of public entities in charge } \\
\text { of the procurement process }\end{array}$ & $\begin{array}{l}\text { Providing advice and comments on } \\
\text { legislation as needed }\end{array}$ \\
\hline PRSP process & $\begin{array}{l}\text { Joint assessment of 2001-2002 } \\
\text { I-PRSP }\end{array}$ & $\begin{array}{l}\text { Aiding in preparation of consultations and } \\
\text { workshops, coordination of donor funding, } \\
\text { recruitment of national experts for } \\
\text { Permanent Secretariat, and the National } \\
\text { Poverty Survey }\end{array}$ & Macro economic aspects \\
\hline
\end{tabular}


Table 3. The Democratic Republic of Congo: Financial Relations with the World Bank GroupStatement of Loans and Credits (in U.S.dollars), as of May 31, 2004.

\begin{tabular}{|c|c|c|c|c|}
\hline & IBRD & IDA & IDA Grant & Total \\
\hline Original principal & $330,000,000$ & $2,773,794,621$ & $460,000,000$ & $3,563,794,621$ \\
\hline Cancellations & $28,484,478$ & $259,852,030$ & 0 & $288,336,508$ \\
\hline Disbursed & $301,515,521$ & $1,838,032,715$ & $74,763,529$ & $2,214,311,765$ \\
\hline Undisbursed & 0 & $867,409,681$ & $398,938,055$ & $1,266,347,736$ \\
\hline Repaid & $247,045,474$ & $192,858,297$ & 0 & $439,903,771$ \\
\hline Due & 0 & $1,828,146,050$ & 0 & $1,828,146,050$ \\
\hline \multirow[t]{2}{*}{ Exchange adjustment } & 0 & 0 & 0 & 0 \\
\hline & & $1,828,146,050$ & & $1,828,146,050$ \\
\hline Borrower's obligation & 0 & & 0 & \\
\hline Sold third party & $54,470,047$ & 0 & 0 & $54,470,047$ \\
\hline Repaid third party & $54,470,047$ & 0 & 0 & $54,470,047$ \\
\hline Due third party & 0 & 0 & 0 & 0 \\
\hline
\end{tabular}




\section{Democratic Republic of the Congo: Statistical Issues}

Despite a difficult environment, the authorities have continued to produce an array of economic and financial statistics, most of which are contained in the Annual Report of the Central Bank of Congo (BCC), which also issues a monthly Statistical Bulletin. Moreover, a comprehensive set of external debt statistics is compiled by the Office de Gestion de la Dette Publique (OGEDEP). Statistical issues related to specific sectoral areas are described below.

Following a fact-finding mission to Kinshasa in June 2001 in real sector and government finance statistics, STA provided technical assistance on government finance statistics in March-April and September 2002, which was augmented by capacity-building support from the World Bank. A STA multi-topic mission visited Kinshasa in October 2003 to assess the DRC's economic and financial data and propose measures to further improve them.

On April 24, 2004, the DRC began participating in the IMF's General Data Dissemination System (GDDS) and its metadata (comprehensive information on statistical production, dissemination practices, and plans for improvement) were posted to the IMF's Dissemination Standards Bulletin Board. Participation in the GDDS will provide a framework for statistical development and capacity building in macroeconomic statistics and sociodemographic (population, health, education, and poverty) indicators.

\section{National accounts}

The national accounts are compiled in constant and current prices by the Directorate of Research of the BCC and published on an annual basis. The compilation methodology conforms to the System of National Accounts 1968 (SNA 1968) and is based on the balance sheets of enterprises and the results of surveys of public and semipublic enterprises and agencies, with most of these surveys dating from the late 1980s. The activities of the traditional sector (including the informal sector) are also included, using extrapolation techniques based on industry-specific data.

\section{Employment and unemployment}

Annual data on employment in the central government are available from the Ministry of Economy, Finance, and the Budget, together with data on employment in the formal sector.

\section{Prices}

Consumer price indices are calculated for Kinshasa by the BCC, the National Statistics Institute (INS), the Institute of Economic and Social Research (IESR), and the Economics Section of the U.S. Embassy in Kinshasa. The IESR also calculates a monthly consumer price index for the Lumumbashi market. The household surveys on which these calculations are 
based date back to the late 1980s and need to be updated to take account of changes in household consumption patterns and demographic shifts.

\section{Government accounts}

The BCC produces aggregated monthly statistics on a cash basis based on its own accounting records of the government cash operations it executes. The treasury produces two sets of monthly statistics based on its own records: one relates to the transactions executed through the BCC, the other set attempts to consolidate operations through commercial bank accounts and off-budget operations. These statistics do not rely on an integrated double-entry public accounting system and provide insufficient details about the nature of expenditures owing to problems in the expenditure chain. However, the treasury has started to produce, on a quarterly basis, expenditure data reports broken down by ministry and institution.

The ongoing improvements in tax administration and expenditure control are expected to improve the quality and timeliness of fiscal statistics. In parallel with technical assistance in the public expenditure management area, STA has been providing technical assistance in government finance statistics on a peripatetic basis, producing significant improvements in the overall quality of government finance statistics. Furthermore, the adoption in June 2004 of a simplified, double-entry accounting framework for the government should significantly improve the availability of data on government financial operations.

In 2003, the DRC reported data for the Government Finance Statistics Yearbook in the format of the Government Finance Statistics Manual 2001.

\section{Monetary accounts}

The Directorate of Research of the BCC regularly produces timely monetary statistics. Generally, the reliability of these statistics is now satisfactory, but some problems remain with the sectorization of accounts.

Since the last money and banking statistics mission in June 2000, data reporting by the BCC for publication in International Financial Statistics (IFS) has resumed; currently, monetary data are reported to STA on a timely basis. The multisector statistics mission that visited Kinshasa in October 2003 recommended a new chart of accounts for the BCC and the other deposit-taking institutions, including information needed for the sectorization of economic units and classification of financial assets.

\section{Balance of payments}

The balance of payments statistics are prepared on an annual basis, based on information on exports and imports of large public and semipublic enterprises, the BCC's payments records, and a survey of residents' foreign operations. The estimates also take account of information on the informal sector, and foreign aid flows provided by the World Bank and the local United Nations Development Program (UNDP) office, which collects these data from the 
European Union, embassies, and nongovernmental organizations. However, the recent multisector mission found that, due to computer problems, data have not been processed since 1999. As a result, the balance of payments is currently compiled using data from different sources, supplemented by estimates.

The mission suggested that the BCC implement a system of quarterly surveys of those corporations that are authorized to hold accounts overseas. Further, the mission recommended the reinvigoration of a working group comprising staff of the Customs and other relevant agencies to prepare quarterly foreign trade data and other measures to improve data on services and transfers. No data are reported to STA for publication.

\section{External and domestic debt}

External and domestic debt statistics are compiled by OGEDEP and are of reasonable quality despite limited computer facilities. However, data on public-enterprise foreign debt and, in particular, on cross-arrears in the public sector, are still of very poor quality. The World Bank is providing assistance in the compilation of cross-arrears in the public sector and public sector arrears with the private sector.

\section{Public enterprise sector}

There is no centralized, comprehensive database on the operations of public enterprises, although IMF missions are provided with some information on the operations of individual enterprises. Generally, data are provided on an annual basis and become available with at least a six-month delay. As part of public enterprise reform, the World Bank is collecting data pertaining to the sector.

\section{Social indicators}

The most consistent data sets are those assembled for the UNDP human development, poverty, and gender-related development indices. Two multiple indicator cluster surveys carried out between 1996 and 2001 in collaboration with the United Nations Children Fund (UNICEF) also provide important social indicators. A national household living standards survey with the help of IDA and other institutions is planned. In addition, in the context of the interim poverty reduction strategy paper (I-PRSP), the authorities, with assistance from the World Bank and the UNDP, have initiated work to construct a comprehensive database for social indicators. 
Democratic Republic of the Congo: Core Statistical Indicators

(As of June 15, 2004)

\begin{tabular}{|c|c|c|c|c|c|c|c|c|c|c|c|}
\hline $\begin{array}{c}\text { Exchange } \\
\text { Rates }\end{array}$ & $\begin{array}{c}\text { International } \\
\text { Reserves }\end{array}$ & $\begin{array}{c}\text { Reserve/ } \\
\text { Base } \\
\text { Money }\end{array}$ & $\begin{array}{l}\text { Central } \\
\text { Bank } \\
\text { Balance } \\
\text { Sheet }\end{array}$ & $\begin{array}{l}\text { Broad } \\
\text { Money }\end{array}$ & $\begin{array}{c}\text { Interest } \\
\text { Rates }\end{array}$ & $\begin{array}{l}\text { Consumer } \\
\text { Price index }\end{array}$ & $\begin{array}{l}\text { Exports } \\
\text { and } \\
\text { Imports }\end{array}$ & $\begin{array}{l}\text { Current } \\
\text { Account } \\
\text { Balance }\end{array}$ & $\begin{array}{c}\text { Overall } \\
\text { Govern- } \\
\text { ment } \\
\text { Balance }\end{array}$ & $\begin{array}{l}\text { National } \\
\text { Accounts }\end{array}$ & $\begin{array}{c}\text { External } \\
\text { Debt }\end{array}$ \\
\hline
\end{tabular}

\begin{tabular}{|c|c|c|c|c|c|c|c|c|c|c|c|c|}
\hline $\begin{array}{l}\text { Date of latest } \\
\text { Observation }\end{array}$ & $05 / 28$ & $04 / 30$ & April 2004 & April 2004 & April 2004 & $05 / 28$ & $05 / 28$ & 2002 & 2002 & $03 / 31 / 04$ & 2003 & Dec. 2002 \\
\hline Date received & $05 / 28$ & $05 / 04$ & $06 / 14 / 04$ & $06 / 14 / 04$ & $06 / 14 / 04$ & $06 / 04$ & $06 / 04$ & $6 / 7 / 03$ & $6 / 7 / 03$ & $04 / 15 / 04$ & $06 / 04$ & $03 / 31 / 03$ \\
\hline Frequency of data $1 /$ & $\mathrm{D}$ & M & M & $\mathrm{M}$ & M & M & $\mathrm{M}$ & A & A & $\mathrm{M}$ & A & A \\
\hline $\begin{array}{l}\text { Frequency of reporting } \\
1 /\end{array}$ & $\mathrm{D}$ & M & M & $\mathrm{M}$ & M & $\mathrm{M}$ & $\mathrm{M}$ & $\mathrm{V}$ & $\mathrm{V}$ & $\mathrm{M}$ & $\mathrm{V}$ & $\mathrm{V}$ \\
\hline Source of data $2 /$ & $\mathrm{N}$ & A & A & A & A & A & A & A & A & A & A & A \\
\hline Mode of reporting 3/ & $\mathrm{E}$ & E & E & E & E & $\mathrm{C}$ & $\mathrm{C}$ & $\mathrm{V}$ & $\mathrm{V}$ & E & V & $\mathrm{V}$ \\
\hline Confidentiality 4/ & $\mathrm{C}$ & A & A & A & A & A & A & A & A & A & A & A \\
\hline $\begin{array}{l}\text { Frequency of } \\
\text { publication } 1 /\end{array}$ & D & $\mathrm{M}$ & $\mathrm{M}$ & $\mathrm{M}$ & $\mathrm{M}$ & $\mathrm{M}$ & $\mathrm{M}$ & A & A & $\mathrm{M}$ & A & A \\
\hline
\end{tabular}

1/ Frequency of data, reporting, and publication: $\mathrm{D}=$ daily, $\mathrm{W}=$ weekly, $\mathrm{M}=$ monthly, $\mathrm{Q}=$ quarterly, $\mathrm{A}=$ annually, or $\mathrm{V}=\mathrm{variable}$

2/ Source of data: $\mathrm{A}=$ direct reporting by central bank, Ministry of Finance, or other official agency, or $\mathrm{N}=\mathrm{official}$ publication or press release.

3/ Mode of reporting: $\mathrm{E}=$ electronic data transfer, $\mathrm{C}=$ cable or facsimile, $\mathrm{T}=$ telephone, $\mathrm{M}=$ mail, $\mathrm{V}=$ staff visits, or $\mathrm{O}=$ other.

4/ Confidentiality: $\mathrm{A}=$ for use by staff only, $\mathrm{B}=$ for use by the staff and the Executive Board, or $\mathrm{C}=$ for unrestricted use 


\section{Statement by the IMF Staff Representative \\ July 12, 2004}

1. The contents of this supplement reflect preliminary information obtained after the finalization of the staff report and does not alter the thrust of the staff appraisal.

2. On the political side, the situation in the east of the country has remained relatively calm since early June but potentially volatile. Most of the Congolese who fled to Rwanda and Burundi when the unrest started have returned. Occasional skirmishes continue to be reported. At end-June 2004, President Kabila appointed the national and provincial commanders of the new integrated national police. On June 28, the law on the new national army was adopted by the National Assembly. It is reported that at their meeting in Nigeria on June 24, 2004, the Heads of State of the DRC and Rwanda agreed on joint control of their border region in collaboration with the United Nations Observation Mission in the DRC (MONUC) and the African Union, as well as on full implementation of the July 2002 Pretoria agreement between the two countries on the withdrawal or expulsion of armed groups from the DRC.

3. On the economic front, preliminary data for the first four months of 2004 point to favorable developments in key indicators, suggesting that real GDP growth may well exceed the program projection of 6.3 percent in 2004 . The cumulative rate of inflation-2.7 percent at end-June 2004 - has remained well in line with the program target of 6 percent for the year. The exchange rate of the Congo franc has continued to be stable. The intervention of the Central Bank of the Congo (BCC) in the foreign exchange market continued to be timid in the second quarter of 2004, with zero net purchases.

4. On the monetary side, preliminary data for key aggregates through end-May 2004 are favorable, reflecting somewhat better-than-programmed budget execution as well as the disbursement of budget support by the World Bank in May 2004, and the quantitative indicative targets for end-June 2004 are likely to have been met. The preliminary results of the external audit of the three end-March 2004 quantitative performance criteria show that the margins by which net foreign assets (NFA), net domestic assets (NDA), and net credit to the government $(\mathrm{NCG})$ have been missed are slightly lower than indicated in the staff report.

5. On the fiscal side, preliminary data for April-May 2004 show a higher domestic primary surplus than programmed (by 0.2 percent of GDP), largely reflecting higher tax revenue and slightly lower current primary expenditure. At end-May, net credit to the government appears to be well within the program target. In light of the recent events in the east of the country, the authorities have reassured the staff that they are taking all necessary actions to adhere to the program objectives, including maintaining security expenditures within their budget allocations.

6. All the three prior actions have been met, i.e. adoption of the 2004 supplementary budget law with main broad aggregates in line with the Memorandum on Economic and 
Financial Policies (MEFP), the Anticorruption Law, and the new Customs Code (MEFP, para. 80).

7. The implementation of structural reforms is progressing well. The National Assembly adopted the law on money laundering and the financing of terrorism on July 5, 2004. The final versions of six tax laws were adopted by Parliament in early July, including keeping the turnover tax rate on services at 18 percent (MEFP, para. 8). However, the government has decided to reserve the right to change by decree the turnover tax rate on "goods of social and strategic importance." On the expenditure side, all expenditure items not channeled through the new expenditure procedures were reincorporated through April 2004, and the budget tracking statements (ESB) for April-May will be produced by mid-July. Finally, the protocols regarding incorporation in the expenditure system of payroll, public debt, and external resources were signed on July 8.

8. The DRC has remained current on its external debt service obligations through endJune 2004. 
Press Release No. 04/145

FOR IMMEDIATE RELEASE

July 13, 2004
International Monetary Fund

Washington, D.C. 20431 USA

\section{IMF Completes Fourth Review Under the Democratic Republic of the Congo's PRGF Arrangement and Grants Additional Interim Assistance Under the Enhanced Initiative for Heavily Indebted Poor Countries}

The Executive Board of the International Monetary Fund (IMF) has completed the fourth review of the Democratic Republic of the Congo's (DRC) performance under a SDR 580 million (about US\$861 million) Poverty Reduction and Growth Facility (PRGF) arrangement (see Press Release No. 02/27). The completion of the review enables the release of a further SDR 26.7 million (about US $\$ 40$ million), which will bring the total amount drawn under the arrangement to SDR 527 million (about US\$782 million).

In completing the review, the Board waived the nonobservance of performance criteria pertaining to the floor on the net foreign assets of the Central Bank of the Congo (BCC), the ceiling on net domestic assets of the BCC, and the ceiling on net bank credit to the government. The Board also concluded that progress on the development of DRC's full Poverty Reduction Strategy Paper (PRSP), as evidenced by the PRSP Preparation Status Report, is satisfactory and provides a sound basis for continued access to IMF concessional assistance.

The Board also approved the authorities' request for additional interim assistance of SDR 1.131 million (about US\$1.7 million) under the enhanced HIPC Initiative.

Following the Executive Board's discussion on the Democratic Republic of the Congo's economic performance, Anne Krueger, First Deputy Managing Director and Acting Chair, stated:

"The Congolese authorities are to be commended for their efforts to implement successfully the PRGF-supported program despite on-going security problems, and to achieve effective reunification, notably through the appointment of provincial governors, the preparation of a national Demobilization, Disarmament, and Reintegration program, and the drawing up of a tentative calendar for elections in the second half of 2005.
"Nonetheless, major challenges remain in demobilizing combatants and successfully holding elections, together with rehabilitating administrative capacity in the reunified provinces and adopting and implementing an effective fiscal decentralization law. The international community needs to continue to assist the authorities in their efforts with timely and well-coordinated support. 
"The satisfactory implementation of fiscal and monetary policies under the program, accompanied by good progress on the structural side, despite some delays, has enabled the country to move from stabilization to reconstruction, as envisaged in its Interim Poverty Reduction Strategy Paper (I-PRSP). However, in the period leading up to the elections in the second half of 2005, the authorities need to maintain their good coordination of economic and financial policies and the momentum of structural reform.

"The authorities have formulated a sound set of priorities for 2004, namely, the implementation of a fiscal decentralization plan, demobilization of soldiers, the maintenance of a relatively constant level of security spending while boosting pro-poor spending, and the continued building up of foreign reserves. On the structural side, the authorities intend to focus on improving governance, including through implementing a law to combat money laundering and passage of a law on corruption, the restructuring of public enterprises and the banking system, and the settlement of the government's net domestic cross-arrears with the private sector, supported by the recently approved supplementary budget.

"To bolster the fiscal position, the authorities should continue to implement comprehensive tax reform to broaden the tax base and improve tax administration. Public expenditure management will benefit from the use of the new budget classification and better procedures to control spending.

"On fiscal decentralization, the authorities have developed a set of principles to ensure fiscal controls at the provincial level that include requiring a balanced budget and constraints on debt issuance by provincial governments. The volatile security situation in the eastern part of the country represents a serious risk to the program, particularly as regards the achievement of fiscal and poverty reduction targets. The forthcoming Regional Peace Conference for the Great Lakes Region could be critical to helping stabilize this region.

"The Central Bank of the Congo (BCC) should continue to aim at price stability within the framework of the floating exchange rate system. In this context, monetary policy instruments should be further strengthened to allow, inter alia, the build up of foreign reserves to a comfortable level. Restructuring of the central bank and commercial banking system is progressing and needs to be sustained.

"The authorities are making efforts to promote a healthy private sector, and include most notably the forthcoming external audit of the diamond sector. These efforts need to be accompanied by measures to ensure good governance and transparency in natural resource activities in accordance with the recently adopted investment, mining, and forestry codes.

"Despite some delays, overall progress toward completing a full high-quality PRSP by August 2005 has been satisfactory and warrants continued support,” Ms. Krueger said.

The PRGF is the IMF's concessional facility for low-income countries. PRGF-supported programs are based on country-owned poverty reduction strategies adopted in a participatory process involving civil society and development partners and articulated in a Poverty Reduction 
Strategy Paper (PRSP). This is intended to ensure that PRGF-supported programs are consistent with a comprehensive framework to foster growth and reduce poverty. PRGF loans carry an annual interest rate of 0.5 percent and are repayable over 10 years with a $5 \frac{1}{2}$-year grace period on principal payments. 